TRANSACTIONS OF THE

AMERICAN MATHEMATICAL SOCIETY

Volume 353, Number 12, Pages 4863-4897

S 0002-9947(01)02853-7

Article electronically published on July 17, 2001

\title{
A MEASURABLE CARDINAL WITH A CLOSED UNBOUNDED SET OF INACCESSIBLES FROM $o(\kappa)=\kappa$
}

\author{
WILLIAM MITCHELL
}

\begin{abstract}
We prove that $o(\kappa)=\kappa$ is sufficient to construct a model $V[C]$ in which $\kappa$ is measurable and $C$ is a closed and unbounded subset of $\kappa$ containing only inaccessible cardinals of $V$. Gitik proved that $o(\kappa)=\kappa$ is necessary.

We also calculate the consistency strength of the existence of such a set $C$ together with the assumption that $\kappa$ is Mahlo, weakly compact, or Ramsey. In addition we consider the possibility of having the set $C$ generate the closed unbounded ultrafilter of $V$ while $\kappa$ remains measurable, and show that Radin forcing, which requires a weak repeat point, cannot be improved on.
\end{abstract}

\section{INTRODUCTION}

In 2, Gitik used a cardinal $\kappa$ with $o(\kappa)=\kappa+1$ to give a forcing construction of a model $V[C]$ in which $\kappa$ is measurable, while the set $C$ is closed and unbounded in $\kappa$ and contains only inaccessibles of $V$. Gitik also showed that no such construction is possible unless $o(\kappa) \geq \kappa$ in $K$. The main result of this paper is that the lower bound $o(\kappa)=\kappa$ is correct:

Theorem 1.1. The following are equiconsistent:

1. There is a cardinal $\kappa$ with $o^{K}(\kappa)=\kappa$.

2. There is a measurable cardinal $\kappa$ and a closed unbounded subset $C$ of $\kappa$ such that each member of $C$ is inaccessible in $K$.

We begin with a proof of Gitik's result that 1.1(2) implies that $o(\kappa)=\kappa$ in the core model $K$. This proof is essentially the same as that of Gitik, but is reorganized to feature the Prikry generic sequence more prominently. Afterward we will reexamine the proof to gain some further insight into the general problem. The proof depends on the following lemma:

Lemma 1.2. Suppose that $\vec{\kappa}=\left(\kappa_{n}: n<\omega\right)$ is K-generic for Prikry forcing by a measure $U \in K$ on $\kappa$, and suppose that each $\kappa_{n}$ is inaccessible and has a closed and unbounded subset $C_{n}$ containing only cardinals inaccessible in $K$. Then $o^{K}(\kappa) \geq \kappa$.

In order to see that the lemma implies the direction $(2) \Longrightarrow(1)$ of theorem 1.1 let $\kappa$ and $C$ be as in clause 1.11(2). Using the measure on $\kappa$, force to add a Prikry sequence $\left(\kappa_{n}: n<\omega\right)$ cofinal in $\kappa$. For all but finitely many $n \in \omega$, the set $C_{n}=$ $C \cap \kappa_{n}$ is a closed and unbounded subset of $\kappa_{n}$ containing only inaccessibles of $K$, and hence the lemma implies that $o^{K}(\kappa) \geq \kappa$.

Received by the editors March 30, 2001.

2000 Mathematics Subject Classification. Primary 03E35, 03E45, 03E55.

This work was partially supported by grant number DMS-962-6143 from the National Science Foundation. 
Proof of lemma 1.2. Assume, towards a contradiction, that $\delta=o^{K}(\kappa)<\kappa$.

In order to allow a generalization of the proof, we will not use the assumption that $\vec{\kappa}$ is a Prikry sequence until the end of the proof.

We begin by applying the covering lemma [3]. Let $X$ be a precovering set such that $\left(\kappa_{n}: n<\omega\right) \in X$ and $\left(C_{n}: n \in \omega\right) \in X$ and such that $X$ contains all of its limit ordinals of cofinality at most $\delta$. The assertion that $X$ is a precovering set means that $|X|<\kappa$, that $X \prec V_{\tau}$ for some $\tau>\kappa=\sup \kappa_{n}$, and that $X$ satisfies a certain closure condition. The covering lemma asserts that such a precovering set exists, and that if $X$ is any such precovering set, then there is a function $h \in K$, an ordinal $\rho<\kappa$, and a system $\mathcal{C}^{X}$ of indiscernibles for $K$ with the following properties, where we write $\mathcal{C}^{X}(\lambda)$ for $\bigcup_{\beta<o^{K}(\lambda)} \mathcal{C}^{X}(\lambda, \beta)$.

1. $\operatorname{domain}(\mathcal{C})=\left((\alpha, \beta) \in X: \beta<o^{K}(\alpha)\right)$, and $\mathcal{C}(\alpha, \beta) \subset\left\{\nu \in \alpha \cap X: o^{K}(\nu)=\right.$ $\beta\}$.

2. If $\nu \in X \backslash \rho$, then either $\nu \in h^{\text {" }} \nu$ or else $\nu \in \mathcal{C}^{X}(\lambda)$ where $\lambda=\inf (h " \nu \backslash \nu) \leq \kappa$.

3. For any $\lambda \in X, \mathcal{C}^{X}(\lambda)$ is closed in $X \cap \lambda$.

4. If $\beta_{0}<o^{K}(\lambda)$ and $\nu$ is a ordinal of cofinality $\omega$ which is a limit point of $\bigcup_{\beta \geq \beta_{0}} \mathcal{C}^{X}(\lambda, \beta)$, then $\nu \in \mathcal{C}^{X}(\lambda, \beta)$ for some $\beta>\beta_{0}$.

5. If $\nu \in \mathcal{C}^{X}(\lambda) \cup\{\lambda\}$ and $\mathcal{C}^{X}(\lambda)$ is bounded in $X \cap \nu$, then there is $\eta \in X \cap \nu$ such that $h$ " $\eta$ is cofinal in $X \cap \nu$.

We can assume, without loss of generality, that $\rho<\kappa_{0}$. For each $n<\omega$, let $\lambda_{n}$ be the least member of $X \cap h{ }^{\prime \prime} \kappa_{n}$. Then $\kappa_{n} \leq \lambda_{n} \leq \kappa$, and if $\lambda_{n}>\kappa_{n}$, then $\kappa_{n} \in \mathcal{C}^{X}\left(\lambda_{n}\right)$.

The proof of lemma 1.2 relies on two observations:

Claim 1.3. 1. For each $n<\omega$, the set $\mathcal{C}^{X}\left(\lambda_{n}\right)$ is cofinal in $\kappa_{n} \cap X$.

2. If $X$ contains all of its limit points of cofinality at most $o^{K}\left(\lambda_{n}\right)$, then $\mathcal{C}^{X}\left(\lambda_{n}\right)$ is bounded in $\kappa_{n} \cap X$.

Proof of claim. For clause 1, suppose to the contrary that $\mathcal{C}^{X}\left(\lambda_{n}\right)$ is bounded in $X \cap \kappa_{n}$. It follows by property 5 of the precovering set that there is $\eta \in X \cap \kappa_{n}$ so that $h^{\prime \prime} \eta$ is cofinal in $X \cap \kappa_{n}$. Let $A$ be the set of limit points of $X \cap h$ " $\eta$. Then $A$ is closed and unbounded in $\kappa_{n}^{\prime}=\sup \left(X \cap \kappa_{n}\right)$, which has uncountable cofinality since $X$ contains all of its limit points of cofinality $\omega$. Hence $A \cap C_{n}$ is closed and unbounded in $\kappa_{n}^{\prime}$; but this is impossible since the $\omega^{\text {th }}$ member $\tau$ of $A \cap C_{n}$ is singular in $K$, since $h " \eta$ is cofinal in $\tau$, contradicting the assumption that every member of $C_{n}$ is inaccessible in $K$.

To prove clause 2, let $\tau=o^{K}\left(\lambda_{n}\right)$ and define a sequence of ordinals $\eta_{\xi}$ for $\xi \leq \tau \cdot \omega$, as follows:

$$
\eta_{\xi}= \begin{cases}0 & \text { if } \xi=0, \\ \sup _{\xi^{\prime}<\xi} \eta_{\xi^{\prime}} & \text { if } \xi \text { is a limit ordinal, } \\ \inf \left(\mathcal{C}^{X}\left(\lambda_{k}, \iota\right) \backslash \eta_{\xi-1}\right) & \text { if } \xi=\tau \cdot k+\iota+1 \text { and } \mathcal{C}^{X}\left(\lambda_{k}, \iota\right) \not \subset \eta_{\xi}, \\ \eta_{\xi-1} & \text { otherwise. }\end{cases}
$$

Since $X$ contains all of its limit points of cofinality at most $\tau$, each of the ordinals $\eta_{\xi}$ is in $X$ for $\xi \leq \tau \cdot \omega$. Furthermore, all of the ordinals $\eta_{\xi}$ are less than $\kappa_{n}$ : The ordinals $\eta_{0}$ and $\eta_{\xi+1}$ are less than $\kappa_{n}$ by definition, while if $\xi$ is a limit ordinal then either $\eta_{\xi}=\eta_{\xi^{\prime}} \neq \kappa_{n}$ for some $\xi^{\prime}<\xi$, or else $\eta_{\xi}$ is singular while $\kappa_{n}$ is regular.

Thus $\eta=\eta_{\tau \cdot \omega}$ is in $\mathcal{C}\left(\lambda_{n}, \beta\right)$ for some $\beta<o^{K}\left(\lambda_{n}\right)$. For each $k<\omega$ it follows that if $\xi_{k}=\tau \cdot k+\beta$ then $\mathcal{C}\left(\lambda_{n}, \beta\right) \backslash \eta_{\xi_{k}} \neq \varnothing$ and hence $\eta_{\xi_{k}}<\eta_{\xi_{k}+1} \in \mathcal{C}\left(\lambda_{n}, \beta\right)$. 
It follows that $\eta_{\tau \cdot \omega}$ is a limit point of $\mathcal{C}\left(\lambda_{n}, \beta\right)$, but this is impossible, as it would follow by (3) that $\eta \in \mathcal{C}\left(\lambda_{n}, \beta^{\prime}\right)$ for some $\beta^{\prime}>\beta$.

We can now complete the proof of lemma 1.2 by observing that the assumption that $\vec{\kappa}$ is a Prikry sequence implies that $\lambda_{n}=\kappa$ for all but finitely many $n<\omega$. Thus $o^{K}\left(\lambda_{n}\right)=o(\kappa)=\delta$, but this is impossible by claim 1.3 since the precovering set $X$ was chosen to contain all of its limit points of cofinality at most $\delta$. Hence $o^{K}(\kappa) \geq \kappa$.

1.1. A warm up. Before proving the other direction of theorem 1.1 we state the similar but simpler theorem 1.5 One direction of the proof is given by a second look at the proof of lemma 1.2 while other direction will be used to introduce some of the ideas of the proof of the main theorem in an easier context.

Definition 1.4. We write $E$ for the class of inaccessible cardinals of $K$, and $D$ for the class of cardinals $\lambda \in E$ such that $\left\{\alpha<\lambda: o^{K}(\alpha)=\beta\right\}$ is stationary in $\lambda$ for all $\beta<\lambda$.

Gitik [2, theorem 1.1] proves that $\kappa \in D$ is equiconsistent with the existence of a closed, unbounded set $C \subset E \cap \kappa$, where $\kappa$ is inaccessible. The following theorem shows that this construction cannot be directly iterated to obtain infinitely many such cardinals $\kappa$ :

Theorem 1.5. The following are equiconsistent:

1. There is an infinite increasing sequence $\vec{\kappa}=\left(\kappa_{n}: n<\omega\right)$ of inaccessible cardinals such that for each $n<\omega$ there is a closed unbounded set $C_{n} \subset \kappa_{n} \cap E$.

2. There is an infinite increasing sequence $\vec{\lambda}$ of cardinals $\lambda_{n} \in D$ with $\kappa=$ $\sup \vec{\lambda}=\lim _{n<\omega} o^{K}\left(\lambda_{n}\right)$.

Furthermore, the model in clause (1) can be constructed so that $o^{K}\left(\kappa_{n}\right)=0$ for all $n \in \omega$.

Proof of (1) $\Longrightarrow(2)$. Assume that $\vec{\kappa}$ is a sequence satisfying condition (1) and, as in the proof of lemma 1.2. pick a precovering set $X$ and use it to define the sequence $\vec{\lambda}$. We will show that $\delta=\lim _{n} o^{K}\left(\lambda_{n}\right) \geq \kappa$. Suppose to the contrary that $\delta<\kappa$, and pick a second precovering set $X^{\prime} \supset X$ such that $X^{\prime}$ contains all of its limit points of cofinality at most $\delta$. We now need to apply one further consequence of the covering lemma, which states that if the ordinals $\lambda_{n}^{\prime}$ are defined from $X^{\prime}$ in the same way as the ordinals $\lambda_{n}$ were defined from $X$, then $\lambda_{n}^{\prime}=\lambda_{n}$ for all but finitely many $n<\omega$. Thus there are infinitely many $n<\omega$ such that $X^{\prime}$ contains all of its limit points of cofinality at most $o^{K}\left(\lambda_{n}\right)$. Since this contradicts claim 1.3, the assumption that $\delta<\kappa$ must be false.

To complete the proof we need to show that $\lambda_{n} \in D$. Now $\kappa_{n} \in D$ by Gitik's result. If $\kappa_{n}=\lambda_{n}$ then we are done, so suppose $\kappa_{n}<\lambda_{n}$. Then $\kappa_{n} \in \mathcal{C}\left(\lambda_{n}, \beta\right)$ for some $\beta<o^{K}\left(\lambda_{n}\right)$ so $D \cap \lambda_{n} \in U\left(\lambda_{n}, \beta\right)$. In particular $D \cap \lambda_{n}$ is stationary in $\lambda_{n}$, but this implies that $\lambda_{n} \in D$.

The final sentence asserting that it is possible to have $o^{K}\left(\kappa_{n}\right)=0$ is included in theorem 1.5 less for its intrinsic interest than because its proof introduces a basic new idea needed for the proof of theorem 1.1

This proof shows, in the language of [4, 5], that the sequence $\left(\kappa_{n}: n<\omega\right)$ is an accumulation point sequence for the sequence $\left(\lambda_{n}: n<\omega\right)$. The sequence $\vec{\lambda}$ is determined by $\vec{\kappa}$, modulo finite changes to the sequence. The exception for finite 
changes is critical for obtaining the measure $U^{*}$ on $\kappa$ in the proof of theorem 1.1 as in the ultrapower $\operatorname{ult}\left(V, U_{\kappa, 0}\right)$ there is no suitable candidate for a cardinal $\lambda$ which would have the same relation to $\kappa$ as the cardinals $\lambda_{n}$ have to $\kappa_{n}$.

\section{Definition of THE FORCING}

Before describing the forcing used to prove the other direction of theorems 1.1 and 1.5. we describe some of the conventions used in this paper.

If $p, p^{\prime}$ are forcing conditions, then we write $p^{\prime} \leq p$ to mean that $p^{\prime}$ is stronger than $p$. If $G \subset P$ is generic and $A$ is a set in $V[G]$ such that $V[A]=V[G]$, then we will write $G=\mathfrak{G}(A)$. In our applications the set $G$ is always uniquely determined by $A$.

By analogy with the usual restriction operator $\uparrow$, we use $\downarrow$ for upward restriction: If $\operatorname{domain}(h)=A$ is a set of ordinals, then $h \downarrow \nu=h \uparrow(A \backslash \nu+1)$. Thus $h=h \uparrow(\nu+1) \cup$ $h \mid \nu$.

Since we are using the Prikry property for forcing notions in which the direct extension order $\leq^{*}$ is not closed, we make the following definitions:

Definition 2.1. 1. A structure $\left(P, \leq, \leq^{*}\right)$ satisfies the $\mu$-Prikry property if $\leq$ and $\leq^{*}$ are partial orders with $\leq^{*} \subset \leq$, and for any sequence $\vec{\sigma}$ of fewer than $\mu$ sentences in the forcing language for $(P, \leq)$ and any condition $p \in P$ there is a condition $p^{\prime} \leq^{*} p$ which decides all sentences in $\vec{\sigma}$.

2. $\left(P, \leq, \leq^{*}\right)$ has the Prikry property if it has the 2-Prikry property.

3 . We say that $P$ is $\mu$-closed if every $\leq^{*}$-descending sequence of length less than $\mu$ has a lower bound in $P$.

4. We say that $p \in P$ satisfies the $\mu$-Prikry property (or is $\mu$-closed) if $\left\{p^{\prime} \in P\right.$ : $\left.p^{\prime} \leq{ }^{*} p\right\}$ satisfies the $\mu$-Prikry property (or is $\mu$-closed).

Since none of the forcing notions used in this paper have a forcing order $\leq$ which is even countably closed, the use of the term "closed" to refer exclusively to the direct extension order $\leq^{*}$ should not cause confusion.

Remark 2.2. If $\left(P, \leq, \leq^{*}\right)$ is $\mu$-closed and has the Prikry property, then it has the $\mu$-Prikry property.

We assume throughout the rest of this paper that the GCH holds in $V$, and that $\vec{U}$ is a coherent sequence of measures in $V$. If these additional conditions do not hold in $V$, then we can work in an inner model $L[\vec{U}]$ which does satisfy these conditions, and which still satisfies the hypotheses of theorems 1.1 and 1.5 .

We will write $U_{\lambda, \beta}$ for the measure $U$ on the sequence $\vec{U}$ such that $\operatorname{crit}(U)=\lambda$ and $o(U)=\beta$. We will write $U(\lambda)=\bigcup_{\beta<o(\lambda)} U_{\lambda, \beta}$.

2.1. Introducing the forcing. In the rest of section 2 we will define the forcing for both the main theorem 1.1 and for the warm up theorem 1.5.

The basic partial order which Gitik uses to add a closed unbounded subset of $E$ is $P_{\kappa}[E]$, which has as conditions the set of closed, bounded subsets of $\kappa \cap E$, ordered by end extension. This is the same ordering used in [1] to add a closed unbounded subset of an arbitrary stationary subset $E$ of $\omega_{1}$. It is easy to see that a generic set $G \subset P_{\kappa}[E]$ will always generate a closed and unbounded subset $C=\bigcup G$ of $\kappa \cap E$, and that the problem is to arrange that $\kappa$ is not collapsed; as it is, for example, if $E$ is the set of inaccessibles in the ground model. In order to avoid this problem Gitik uses iterated Prikry-Magidor forcing as a preliminary forcing to 
obtain an intermediate model with enough bounded, closed subsets of $E$ so that $P_{\kappa}[E]$, defined in the intermediate model, is $<\kappa$-distributive. This distributivity ensures that forcing with $P_{\kappa}[E]$ does not collapse cardinals, change cofinalities or add new bounded subsets of $\kappa$.

Thus $P_{\kappa}[E]$ works well for adding a closed unbounded subset of $E \cap \kappa$ for a single cardinal $\kappa$, but it is clear from lemma 1.5 that it cannot be simultaneously applied to infinitely many cardinals $\kappa$. In order to leave $\kappa$ measurable in the generic extension, Gitik defines a variant of $P_{\kappa}[E]$, which we call $Q_{\kappa}^{*}$, for cardinals $\kappa$ with $o(\kappa) \geq \kappa$. The forcing $\left(P_{\kappa}[E], \leq\right)$ is dense in $\left(Q_{\kappa}^{*}, \leq\right)$, so that forcing with $Q_{\kappa}^{*}$ is equivalent to forcing with $P_{\kappa}[E]$, but because $Q_{\kappa}^{*}$ has the Prikry property and is $\kappa$-closed it is possible to iterate it to deal with infinitely many cardinals.

The forcing $Q_{\kappa}^{*}$ is still not quite adequate for our problem. In order to be $\kappa$ closed it requires $o(\kappa) \geq \kappa$, and we only assume this for the single cardinal $\kappa$ which is to remain measurable. However $Q_{\lambda}^{*}$ is $\operatorname{cf}(o(\lambda))$-closed, and under the hypothesis of theorem 1.5 this will allow us to choose, for any $\gamma<\kappa$, a tail of the forcing which is $\gamma$-closed. This is sufficient since distributivity can be used to deal with the lower part of the forcing. The proof that $\kappa$ remains measurable in $V[C]$ does require that $Q_{\kappa}^{*}$ be $\kappa$-closed and hence requires $o(\kappa)=\kappa$.

This still leaves a problem. Theorem 2.13 specifies that $o\left(\kappa_{n}\right)=0$ for each of the regular cardinals $\kappa_{n}$ of $V[C]$ such that $C \cap \kappa_{n}$ is unbounded in $\kappa_{n}$. As was pointed out earlier, this was required primarily to provide an introduction to the idea of the proof of theorem 1.1 where, because the desired measure $U^{*}$ on $\kappa$ in $V[C]$ extends the order zero measure $U_{\kappa, 0}$ in $K$, we have $o^{K}(\kappa)=0$ in $M\left[C^{*}\right]=\operatorname{ult}\left(V[C], U^{*}\right)$. Thus, if $Y$ is the set of of inaccessible limit points $\lambda$ of $C$ such that $o^{K}(\lambda)=0$ then $Y \in U^{*}$. Since we will use our modification of $P_{\lambda}[E]$ for each $\lambda \in Y$, we have to deal with the restriction that $P_{\lambda}[E]$ can only be used at finitely many cardinals $\lambda$. The solution to this problem is suggested by Prikry forcing: a condition $p$ contains only finitely many instances of the forcing $P_{\lambda}[E]$, but it also contains instances $(\gamma, B, q)$ of a new forcing notion $U_{\gamma, 0} \times Q_{\gamma}^{*}$. The set $B \in U_{\gamma, 0}$ is used, as in Prikry forcing, to specify the set of cardinals $\lambda$ at which an instance of $P_{\lambda}[E]$ may eventually be added. Until this time, the $\operatorname{cf}(o(\gamma))$-closed condition $q \in Q_{\gamma}^{*}$ plays the role, uniformly for all $\lambda \in B$, of the future instance of $P_{\lambda}[E]$.

The formal definition of the forcing will be given in subsection 2.2 below. The rest of this subsection 2.1 gives an informal description which includes a discussion of our general framework for iterated forcing, an introduction to our notation and ideas, and a brief survey of the four recipes which make up the forcing. It also contains a number of half truths: in particular, it glosses over the fact that the forcing is a backward Easton forcing, and mentions an important side condition $\vec{B}$ only briefly .

We have chosen to present the forcing in a framework somewhat different from that used by Gitik. We do not use Gitik's preliminary forcing; instead the closed sets needed to make the forcing distributive are incorporated, when needed, into the main forcing. The final model is $V[C]$, and as with Prikry-Magidor forcing it has the property that $\mathcal{P}^{V[C]}(\lambda) \subset V[C \cap \lambda]$ for any $\lambda<\kappa$. In particular, cofinalities are not changed except at limit points of $C$.

Partly as a benefit of this modification, this paper is self-contained and does not assume a previous understanding of Gitik's papers.

Our approach is suggested by a comparison of $P_{\kappa}[E]$ and Prikry-Magidor-Radin forcing. Recall that a Prikry-Magidor-Radin condition to add a subset of $\kappa$ is a 
finite sequence of the form

$$
\left(\left(\gamma_{0}, A_{\gamma_{0}}\right), \ldots\left(\gamma_{n-1}, A_{\gamma_{n-1}}\right),\left(\gamma_{n}, A_{\gamma_{n}}\right)\right)
$$

where $\gamma_{0}<\cdots<\gamma_{n-1}<\gamma_{n}=\kappa$ and each set $A_{\gamma_{i}}$ is a member of $U\left(\gamma_{i}\right)=$ $\bigcap_{\beta<o\left(\gamma_{i}\right)} U_{\gamma_{i}, \beta}$. The direct extension ordering $\leq^{*}$ is defined by $p^{\prime} \leq^{*} p$ if $p^{\prime}=$ $\left(\left(\gamma_{0}, A_{\gamma_{0}}^{\prime}\right), \ldots,\left(\gamma_{n-1}, A_{\gamma_{n-1}}^{\prime}\right),\left(\kappa, A_{\kappa}^{\prime}\right)\right)$ has the same domain and $A_{\gamma_{i}}^{\prime} \subset A_{\gamma_{i}}$ for each $i \leq n$. The forcing order $p^{\prime} \leq p$ includes the additional non-direct extension in which $p^{\prime}$ has new pairs $\left(\gamma, A_{\gamma_{k}} \cap \gamma\right)$, with $\gamma_{k-1}<\gamma<\gamma_{k}$, such that $\gamma \in A_{\gamma_{k}}$ and $A_{\gamma_{k}} \cap \gamma \in U(\gamma)$.

If $G$ is a generic subset of this forcing, then $C=\bigcup\{$ domain $p: p \in G\}$ is a closed subset of $\kappa+1$ satisfying the following two properties: (i) $C$ is a closed subset of $\kappa+1$ with $\kappa \in C$ and (ii) if $\lambda \in C$ and $A \subset \lambda$ is in the ground model then $C \cap A$ is unbounded in $\lambda$ if and only if $A \in U(\lambda)$. Conversely, any set $C$ satisfying these properties is generic for Prikry-Magidor-Radin forcing.

A condition from either $P_{\kappa}[E]$ or Prikry-Magidor-Radin forcing can be viewed as a pair $p^{\frown}(\kappa, q)$, where $p$ is a term denoting a closed, bounded subset of $\kappa$ and $q$ is a recipe specifying the allowed end extensions of $p$. In the case of $P_{\kappa}[E]$, the term $p$ is a closed set from the ground model and the recipe allows arbitrary end extensions. In the case of a Prikry-Magidor-Radin condition (1), the term $p$ is $\left(\left(\gamma_{0}, A_{\gamma_{0}}\right), \ldots,\left(\gamma_{n-1}, A_{\gamma_{n-1}}\right)\right)$, itself a Prikry-Magidor condition for a closed and unbounded subset of $\gamma_{n-1}$, and the recipe is $A_{\kappa}$, which allows extensions of $p$ consisting of adding pairs $\left(\gamma, A_{\kappa} \cap \gamma\right)$ for cardinals $\gamma \in A_{\kappa} \backslash \gamma_{n-1}+1$.

As in Prikry-Magidor-Radin forcing, a condition in our forcing $\mathcal{R}_{\lambda}$ is a function with a finite domain, which we can write as $p=\left(\left(\gamma_{0}, q_{0}\right), \ldots,\left(\gamma_{k-1}, q_{k-1}\right)\right)$ where $\gamma_{0}<\cdots<\gamma_{k-1}<\lambda$ and $q_{i}$ specifies a recipe for extending $p\left\lceil\gamma_{i}=\left(\left(\gamma_{0}, q_{0}\right), \ldots\right.\right.$, $\left.\left(\gamma_{i-1}, q_{i-1}\right)\right)$. A condition in $\mathcal{R}_{\lambda}$ will have domain contained in $\lambda$. We will use $\mathcal{R}_{\nu, \lambda}$ for the sub-ordering of $\mathcal{R}_{\lambda}$ which adds a closed subset of $\lambda \backslash \nu+1$, using conditions which are (to the extent they are closed at all) $\nu^{+}$-closed. We will write $\mathcal{R} / p$ for the set of conditions $p^{\prime} \leq p$ in $\mathcal{R}_{\lambda}$.

If $G$ is a generic subset of $\mathcal{R} / p$, then $V[G]=V[C]$, where $C$ is the closed subset of $\max (\operatorname{domain} p)+1$ defined by

$$
\begin{aligned}
\gamma \in C & \Longleftrightarrow \exists p^{\prime} \in G \forall p^{\prime \prime} \leq p^{\prime} \gamma \in \operatorname{domain} p^{\prime \prime} \\
& \Longleftrightarrow \exists p^{\prime} \in G\left((\gamma, q) \in p \text { is not an instance of the recipe } U_{\gamma, 0} \times Q_{\gamma}^{*}\right)
\end{aligned}
$$

That is, an instance $(\gamma, q)$ of any of the first three recipes described below will force that $\gamma$ is a member of $C$ and (except in the special case $q \in Q_{\lambda}$ with $o(\lambda)=0$ ) is also a limit point of $C$. The recipe $U_{\gamma, 0} \times Q_{\gamma}^{*}$ is excluded because it does not add a closed and unbounded subset of $\gamma$, but instead serves as a proxy for a future instance of $P_{\gamma^{\prime}}$ for some cardinal $\gamma^{\prime}<\gamma$.

We will write $\lceil p\rceil$ for $\sup (\operatorname{domain} p)$, and $\lfloor p\rfloor$ for a cardinal $\gamma$ (to be defined specifically later) such that $p \Vdash \inf C>\lfloor p\rfloor$. If $p_{0}$ and $p_{1}$ are conditions with $\left\lceil p_{0}\right\rceil<\left\lfloor p_{1}\right\rfloor$, then we can concatenate $p_{0}$ and $p_{1}$ to obtain a third condition: $p=$ $p_{0} \frown p_{1}$. As a minor abuse of notation we will identify a single instance $(\gamma, q)$ of a recipe with the condition $((\gamma, q))$; for example by writing $p_{0} \frown(\gamma, q) \frown p_{1}$.

If $p=p_{0} \frown p_{1}$, then we will write $C_{p_{i}}$ for $C \cap\left(\left\lceil p_{i}\right\rceil+1 \backslash\left\lfloor p_{i}\right\rfloor+1\right)$. Thus $p \Vdash C=$ $C_{p}=C_{p_{0}} \cup C_{p_{1}}$. We will write " $C_{p_{i}}$-term" to mean a term specifying a member of $V\left[C_{p_{i}}\right]$, which is to say a term in the forcing language for $\mathcal{R} / p_{i}$. Note that $\mathcal{R} / p_{i}$ is contained in $\mathcal{R}_{\left\lfloor p_{i}\right\rfloor,\left\lceil p_{i}\right\rceil+1}$. 
If $p=p_{0} \frown p_{1}$, then $\mathcal{R} / p \equiv \mathcal{R} / p_{0} * \dot{\mathcal{R}} / p_{1}$. For conditions with finite length, we can use this factorization to reduce the problem of defining the order on $\mathcal{R}_{\lambda}$ to the case $p \leq(\gamma, q)$ and $p \leq^{*}(\gamma, q)$ where the right hand side is a single instance of one of the recipes. We will assume len $(p)$ is finite through the rest of this subsection.

We now finish the informal description with a survey of the four recipes which we will use.

1. The recipe $P$. An instance of the recipe $P_{\lambda}$ is designated in a condition by the pair $(\lambda, \bar{\lambda})$, with $\bar{\lambda}<\lambda$. This recipe is the analog of Gitik's forcing $P_{\lambda}[E]$ : A condition of the form $p^{-}(\lambda, \bar{\lambda})$ allows as a direct extension any extension of the form

$$
p^{\frown} p^{\prime} \frown\left(\lambda, \bar{\lambda}^{\prime}\right) \leq^{*} p^{\frown}(\lambda, \bar{\lambda})
$$

where $\bar{\lambda}<\bar{\lambda}^{\prime}<\lambda, p^{\prime} \in \mathcal{R}_{\bar{\lambda}, \bar{\lambda}^{\prime}+1}$, and every recipe in $p^{\prime}$ is $\bar{\lambda}$-closed. This latter restriction means in particular that no instance of $P$ can occur in $p^{\prime}$. Such an instance may occur in a condition $p^{-} p^{\prime \prime} \leq(\lambda, \bar{\lambda})$ but such an instance must be spawned in two steps: $p^{\frown} p^{\prime \prime} \leq p^{\frown} p^{\prime} \leq^{*} p \cap(\lambda, \bar{\lambda})$ where the instance of $P$ is spawned by an instance of the $\bar{\lambda}$-closed recipe $U_{\gamma, 0} \times Q_{\gamma}^{*}$ in $p^{\prime}$.

This is the only recipe permitting a direct extension which changes the domain of the condition $p$ of which it is a member. In all other cases direct extensions affect only the instance of the recipe concerned. As a consequence, this is the only recipe for which the direct extension ordering $\leq^{*}$ is not countably closed.

2. The recipe $Q$. This recipe is a generalization of Prikry-Magidor forcing. An instance of $Q_{\lambda}$ is designated by a triple $(\lambda, A, h)$, where $o(\lambda)<\lambda, A \subset E$, $A \in U(\lambda)$, and $h$ is a function such that $h(\nu)$ is a $\nu$-closed condition in $\mathcal{R}_{\nu, \lambda}$ for each $\nu \in A$. The basic non-direct extension of a condition $(\lambda, A, h)$ has the form $\left(\nu, A \cap \nu, h\lceil\nu) \frown h(\nu) \frown(\lambda, A \backslash \nu, h \mid \nu) \leq(\lambda, A, h)\right.$. Thus, if $p=p^{\prime} \frown(\lambda, A, h)$, then $p$ forces that the generic set has the form

$$
C=C_{p^{\prime}} \cup \bigcup_{\nu \in \bar{C}}\left(\{\nu\} \cup C_{h(\nu)}\right) \cup\{\lambda\}
$$

where $\bar{C}$ is a Prikry-Magidor generic subset of $\lambda$ and $C_{h(\nu)}$ is generic for the forcing $\mathcal{R} / h(\nu)$. If $\nu$ and $\nu^{\prime}$ are successive members of $\bar{C}$ then $C_{h(\nu)} \subset\left(\nu, \nu^{\prime}\right)$.

The recipe $Q_{\lambda}$ is our adaptation of the way in which Gitik uses Prikry-Magidor sequences generated by a preliminary forcing to obtain an intermediate model in which $P_{\lambda}[E]$ is $<\lambda$-distributive. This recipe, together with $P_{\lambda}$, is also used as the basis for the remaining recipes. Notice that $\nu$-completeness of $h(\nu)$ implies in particular that $h(\nu)$ does not contain any instance of $P$.

If $\beta<o(\lambda)$ then we write $Q_{\lambda, \beta}$ for the forcing defined exactly like $Q_{\lambda}$, but using only the measures $U_{\kappa, \beta^{\prime}}$ for $\beta^{\prime}<\beta$.

3. The recipe $Q^{*}$. This is our adaptation of the forcing which Gitik uses in order to preserve the measurability of $\kappa$. A instance of the recipe $Q_{\lambda}^{*}$ has one of the two forms $(\lambda, 0, \bar{\lambda})$ or $(\lambda, \beta, A, h)$. An instance of the first form corresponds to an instance $(\lambda, \bar{\lambda})$ of the recipe $P_{\lambda}$ and allows extensions like those given above as (2); however those extensions, unlike those for $P$, are not direct. We will use these instances to view $\left(P_{\lambda}, \leq\right)$ as a dense subset of $\left(Q_{\lambda}^{*}, \leq\right)$.

In the second form, $\beta$ is an ordinal with $0<\beta<o(\lambda)$ and the triple $(\lambda, A, h)$ is an instance of $Q_{\lambda, \beta}$. It has all of the direct extensions of $Q_{\lambda, \beta}$, and in addition it allows direct extensions of the form $\left(\lambda, \beta^{\prime}, A^{\prime}, h^{\prime}\right) \leq^{*}(\lambda, \beta, A, h)$ with $\beta^{\prime}>\beta$ and 
$h^{\prime}(\nu)$ defined arbitrarily for $\beta \leq o(\nu)<\beta^{\prime}$. The basic non-direct extension has the following form:

$$
(\gamma, A \cap \gamma, h\lceil\gamma) \frown(\lambda, 0, \gamma) \leq(\lambda, \beta, A, h)
$$

where $0<\beta<o(\gamma)$ and $(\gamma, A \cap \gamma, h \uparrow \gamma) \in Q_{\gamma}$.

The two forms are related by treating an instance $(\lambda, 0, \bar{\lambda})$ of the first form essentially as an instance $(\lambda, 0, \varnothing, \varnothing)$ of the second form. Thus, as with Gitik's forcing, $\left(P_{\lambda}, \leq\right)$ is dense in $\left(Q_{\lambda}, \leq\right)$. The direct extension order $\leq^{*}$ of $Q_{\lambda}^{*}$ is $\operatorname{cf}(o(\lambda))$ closed; this is a minor, but convenient, emendation of Gitik's definition.

4. The final recipe, $U \times Q^{*}$. This recipe is new to this paper and is the construction which allows us to have the effect of iterating the forcing $P_{\lambda}$ at infinitely many cardinals $\lambda$. An instance of the recipe $U_{\lambda, 0} \times Q_{\lambda}^{*}$ has the form $(\lambda, B, q)$ where $B \in U_{\lambda, 0}$ and $q \in Q_{\lambda}^{*}$. It has direct extensions of the form $\left(\lambda, B^{\prime}, q^{\prime}\right) \leq^{*}(\lambda, B, q)$ where $B^{\prime} \subset B$ and $q^{\prime} \leq^{*} q$ in $Q_{\lambda}^{*}$. It allows any of the non-direct extensions allowed by $Q_{\lambda}^{*}$, and in addition it has the following terminal extension, in which it dies after spawning an instance of $P_{\gamma}$ for some $\gamma<\lambda$ :

$$
(\gamma, \bar{\gamma}) \leq(\lambda, B, 0, \bar{\gamma})
$$

where $\bar{\gamma}<\gamma \in B$. Thus the recipe $U_{\lambda, 0} \times Q_{\lambda}^{*}$ may be viewed as a $\operatorname{cf}(o(\lambda))$-closed proxy for an instance of the recipe $P$.

The forcing notions for theorems 1.1 and 2.13 differ in their use of recipes $U \times Q^{*}$. The maximal condition for theorem 2.13 will contain infinitely many instances $U_{\lambda_{n}, 0} \times Q_{\lambda_{n}}^{*}$ of this recipe; and an arbitrary condition will contain, except for these instances of $U_{\lambda_{n}, 0} \times Q_{\lambda_{n}}^{*}$ and the instances of $P_{\kappa_{n}}$ which they spawn, only instances of the recipe $Q$. In the forcing for the main theorem 1.1, in contrast, instances of $P_{\lambda}$ will give rise to instances of $U_{\gamma, 0} \times Q_{\gamma}^{*}$ for $\gamma<\lambda$, and these in turn will spawn new instances of $P_{\gamma^{\prime}}$ for $\gamma^{\prime}<\gamma$.

This causes a difficulty in the proof of the Prikry property for theorem 1.1 the solution to which requires an additional complication in the definition of the forcing. Suppose that $\sigma$ is a sentence, and we are looking for a condition $p^{\prime} \leq^{*} p$ which decides $\sigma$. If $(\lambda, B, q)$ is any individual instance of $U_{\lambda, 0} \times Q_{\lambda}^{*}$ in the condition $p$, then there are two possible cases. The simplest is that there is $B^{\prime} \subset B$ in $U_{\lambda}$ and $q^{\prime} \leq^{*} q$ such that for all $\gamma \in B^{\prime}$, the sentence $\sigma$ is decided by any non-direct extension of $q^{\prime}$ in which $U_{\lambda, 0} \times Q_{\lambda}^{*}$ spawns an instance of $P_{\gamma}$. In this case there is no problem: after further shrinking $B^{\prime}$ so that $\sigma$ is always decided the same way, the direct extension $\left(\lambda, B^{\prime}, q^{\prime}\right)$ decides $\sigma$.

The other possibility is that there is $B^{\prime} \subset B$ in $U_{\lambda}$ such that if $\gamma \in B^{\prime}$, then $\sigma$ is not decided by a non-direct extension spawning an instance of $P_{\gamma}$. In this case we use the direct extension $\left(\lambda, B^{\prime}, q\right) \leq^{*}(\lambda, B, q)$ to ensure that $\sigma$ is not accidentally decided by a non-direct extension using a cardinal $\gamma \notin B^{\prime}$. The problem is that we must take this step not only for instances of $U_{\gamma, 0} \times Q_{\gamma}^{*}$ occurring in the condition $p$, but for instances of $U_{\gamma^{\prime}, 0} \times Q_{\gamma^{\prime}}^{*}$ which could occur in potential extensions $p^{\prime \prime} \leq p^{\prime}$. This is accomplished by including an additional sequence $\vec{B}$ in the conditions. The domain of $\vec{B}$ is the set of measurable cardinals below $\kappa$, and the values are sets $B_{\gamma} \in U_{\gamma, 0}$. It's effect comes from the requirement that any new instance $(\gamma, B, q)$ of $U_{\gamma, 0} \times Q_{\gamma}^{*}$ in $\mathbf{p}^{\prime \prime}<\mathbf{p}^{\prime}$ must satisfy $B \subset B_{\gamma}$.

The only place where the sequence $\vec{B}$ is used is in the proof of lemma 4.4, which is needed to prove the Prikry property for the forcing for the main theorem. Other 
than this, the sequence $\vec{B}$ has no essential effect on the arguments used to prove theorem 1.5 and the proof of the Prikry property 4.7 for the main theorem consists primarily of a verification that the proof used in the warm up carries over. The effect of the sequence $\vec{B}$ is best seen in the proof of theorem 4.11] stating that $\kappa$ remains measurable, and in the statement and sufficiency proofs of theorem 5.3 generalizing the main results to intermediate large cardinal properties.

2.2. The formal definition of the forcing. In this section we define two notions of forcing: $\mathcal{R}_{\kappa}^{\prime}$, to be used for the warm up theorem 1.5, and $\mathbf{R}_{\kappa+1}$, to be used for the main theorem 1.1 The reader may wish to ignore the more complicated forcing $\mathbf{R}_{\kappa+1}$ the first time through.

Note that the definitions 2.3 and 2.5 of the two forcing notions rely on the later definition 2.6 to define their sets of conditions and on definitions 2.7 and 2.8 to define their orderings. Definitions 2.6 2.8, in turn, rely on the definitions 2.10-2.15 of the individual recipes.

Recall that the hypothesis of theorem 1.5 gives an increasing sequence $\left(\lambda_{n}\right.$ : $n<\omega)$ converging to $\kappa$, with $\kappa=\liminf _{n<\kappa} o\left(\lambda_{n}\right)$.

Definition 2.3 (The forcing $\mathcal{R}_{\kappa}^{\prime}$ for theorem 1.5). A condition in the forcing $\mathcal{R}_{\kappa}^{\prime}$ for the warm up theorem 1.5 is a function $p$ as defined in definition 2.6 below, which is below the maximal condition $\mathbf{1}^{\prime}=\left(\left(\lambda_{n}, B_{n}, 0, \lambda_{n-1}\right): n<\omega\right)$, where $B_{n}=\{\nu \in$ $\left.D \cap\left(\lambda_{n-1}, \lambda_{n}\right): o(\nu)=0\right\}$.

Thus the maximal condition $\mathbf{1}^{\prime}$ in $\mathcal{R}_{\kappa}^{\prime}$ consists of an infinite sequence of instances of the recipe $U_{\lambda_{n}, 0} \times Q_{\lambda_{n}}^{*}$. Every condition in $\mathcal{R}_{\kappa}^{\prime}$ is a sequence of length $\omega$, with domain differing from that of $\mathbf{1}^{\prime}$ only on a finite set.

If $\lambda<\kappa$ then we write $\mathcal{R}_{\lambda}^{\prime}$ for $\left\{p\left\lceil\lambda: p \in \mathcal{R}_{\kappa}^{\prime}\right\}\right.$. Thus every member of $\mathcal{R}_{\lambda}^{\prime}$ is a finite sequence.

Definition 2.4. A measure one sequence $\vec{B}$ on an interval $(\gamma, \lambda)$ is a function with domain $\{\nu \in D \cap(\gamma, \lambda): o(\nu)>0\}$ such that $B_{\gamma} \in U_{\gamma, 0}$ for all $\gamma$ in its domain. We say that $\vec{B}^{\prime} \subset \vec{B}$ if $B_{\gamma}^{\prime} \subset B_{\gamma}$ for all $\gamma$ in their common domain.

Definition 2.5 (The forcing $\mathbf{R}_{\kappa+1}$ for theorem 1.1). The conditions in the forcing $\mathbf{R}_{\kappa+1}$ are pairs $\mathbf{p}=(p, \vec{B})$, where

1. $p$ is a finite function in the set $\mathcal{R}_{\kappa+1}$ from definition [2.6 below.

2. $\vec{B}$ is a measure one sequence on the interval $(0, \kappa)$.

We will say that $\left(p^{\prime}, \vec{B}^{\prime}\right) \leq(p, \vec{B})$ or $\left(p^{\prime}, \vec{B}^{\prime}\right) \leq *(p, \vec{B})$ if $\vec{B}^{\prime} \subset \vec{B}$, and if $p^{\prime}$ and $p$ satisfy definitions 2.7 and 2.8 below (taking into account the sequence $\vec{B}$ ).

The maximal element of $\mathbf{R}_{\kappa+1}$ is $\left(\mathbf{1}, \vec{B}^{\mathbf{1}}\right)$ where $\mathbf{1}=(\kappa, 0)$, a single instance of $P_{\kappa}$, and $B_{\gamma}^{1}=\{\nu \in \gamma \cap D: o(\nu)=0\}$.

Definition 2.6 (The underlying sets of $\mathcal{R}_{\lambda}^{\prime}$ and $\mathcal{R}_{\lambda}$ ). We give a simultaneous definition of the two sets; however it should be noted that, because the terms of the forcings differ, the sets will also differ.

The conditions are sequences of pairs, $p=\left(\left(\dot{\gamma}_{k}, \dot{q}_{k}\right): k<n\right)$ with $n \leq \omega$, such that

1. $\dot{\gamma}_{k}$ is a $C_{p \nmid k}$-term for an ordinal such that $p \nmid k \Vdash \dot{\gamma}_{k-1}<\dot{\gamma}_{k}<\lambda$ for all $k<n$.

2. $\dot{q}_{k}$ is a $C_{p \nmid k}$-term for an instance at $\dot{\gamma}$ of one of the recipes which were described in the last subsection and are defined formally in definitions 2.10 through 2.15 below. 
Note that $\dot{\gamma}_{0}$ and $\dot{q}_{0}$ are terms in the trivial forcing $\mathcal{R} / \varnothing$, thus they are not really terms at all but instead are sets in the ground model.

If $p$ is in $\mathcal{R}_{\lambda}$ then we will write $\lceil p\rceil$ for $\sup (\operatorname{domain} p)$. We will write $\lfloor p\rfloor$ for $\left\lfloor\left(\gamma_{0}, q_{0}\right)\right\rfloor$, where $\left(\gamma_{0}, q_{0}\right)$ is the first member of $p$. The definition of $\left\lfloor\left(\gamma_{0}, q_{0}\right)\right\rfloor$ is given as part of the definitions 2.10 2.15 of the individual recipes.

If $p_{0}$ and $p_{1}$ are in $\mathcal{R}_{\lambda}$ and $p_{0} \Vdash\left\lceil p_{0}\right\rceil<\left\lfloor p_{1}\right\rfloor$, then $p_{0} \frown p_{1}$ is also a condition. More generally, if $p_{0}$ is in $\mathcal{R}_{\lambda}$ and $\dot{p}_{1}$ is a $\mathcal{R} / p_{0}$-term for a member of $\mathcal{R}_{\lambda}^{V\left[C_{p_{0}}\right]}$ such that $p_{0} \Vdash\left\lceil p_{0}\right\rceil<\left\lfloor\dot{p}_{1}\right\rfloor$, then we can regard $p_{0} \frown \dot{p}_{1}$ as a member of $\mathcal{R}_{\lambda}$ provided that the condition $p_{0}$ determines the length of the condition $p_{1}$ denoted by $\dot{p}_{1}$, as well as the recipes used at each coordinate. There are only countably many finite sequences of recipes, and hence the $\omega_{1}$-Prikry property of $\mathcal{R} / p_{0}$, once it has been proved, will imply that there is always a condition $p_{0}^{\prime} \leq{ }^{*} p_{0}$ such that $p_{0}^{\prime}-\dot{p}_{1}$ can be regarded as a condition in $\mathcal{R}_{\lambda}$.

We will frequently omit dots on terms, and particularly on the pairs $\left(\gamma_{k}, q_{k}\right)$ in a condition, unless the distinction is needed to clarify the argument.

Definition $2.7\left(\leq^{*}\right)$. The ordering $\leq^{*}$ is the smallest partial ordering satisfying the following two rules:

1. If $p_{0}^{\prime}, p_{1}^{\prime}$ and $p_{0}$ are finite sequences such that $p_{0}^{\prime} \leq^{*} p_{0}$, and if $\dot{p}_{1}^{\prime} \leq^{*}(\dot{\gamma}, \dot{q})$ according whichever of the definitions 2.10 2.15 is appropriate to the recipe $(\dot{\gamma}, \dot{q})$, then $p_{0}^{\prime} \frown \dot{p}_{1}^{\prime} \leq^{*} p_{0} \frown(\dot{\gamma}, \dot{q})$.

2. If $p^{\prime}$ and $p$ are in $\mathcal{R}_{\kappa}^{\prime}$ then $p^{\prime} \leq^{*} p$ if and only if $p^{\prime}\left\lceil\lambda \leq^{*} p\left\lceil\lambda\right.\right.$ in $\mathcal{R}_{\lambda}^{\prime}$ for every $\lambda<\kappa$.

Note that clause 1 is used for both for $\mathcal{R}_{\lambda}$ and, if $\lambda<\kappa$, for $\mathcal{R}_{\lambda}^{\prime}$. Clause 2 is used only for $\mathcal{R}_{\kappa}^{\prime}$.

Definition $2.8(\leq)$. The ordering $\leq$ on $\mathcal{R}_{\lambda}$ and $\mathcal{R}_{\lambda}^{\prime}$ is the smallest partial ordering determined by the following two rules:

1. We have $p_{0}^{\prime} \frown \dot{p}_{1}^{\prime} \leq p_{0} \frown(\dot{\gamma}, \dot{q})$ whenever $p_{0}^{\prime} \leq p_{0}$, and $p_{0}^{\prime} \Vdash \dot{p}_{1}^{\prime} \leq(\dot{\gamma}, \dot{q})$ under whichever of definitions 2.10$] 2.15$ is appropriate to the recipe of which $(\dot{\gamma}, \dot{q})$ is an instance.

2. If $p^{\prime}$ and $p$ have length $\omega$ then $p^{\prime} \leq p$ if and only if there is some $\lambda<\operatorname{len}(p)$ such that $p^{\prime}|\lambda \leq p| \lambda$ and $p^{\prime}\left|\lambda \leq^{*} p\right| \lambda$.

Again, clause 2 applies only to $\mathcal{R}_{\kappa}^{\prime}$.

In the case of $\mathbf{R}_{\lambda}$, the measure one sequence will affect the orderings $p \leq(\dot{\gamma}, \dot{q})$ and $p \leq^{*}(\dot{\gamma}, \dot{q})$, as specified in definition 2.15

In all cases, the relation $p^{\prime} \leq p$ implies $\sup \left(\right.$ domain $\left.p^{\prime}\right) \leq \sup (\operatorname{domain} p)$. It follows that, as far as the forcing order $\leq$ is concerned, we can assume that domain $p$ is determined for all conditions $p$ :

Proposition 2.9. For any condition $p$ there is $p^{\prime} \leq p$ such that all members of domain $p^{\prime}$ are determined, that is, if $p^{\prime}=\left(\left(\dot{\gamma}_{i}, \dot{q}_{i}\right): i<n\right)$, then there are ordinals $\gamma_{i}$ such that $p^{\prime}\left\lceil i \Vdash \dot{\gamma}_{i}=\check{\gamma}_{i}\right.$ for each $i<n$.

Proof. If $p \leq \mathbf{1}^{\prime}$ in the forcing for lemma 1.5, then all but finitely many members of domain $(p)$ come from domain $\left(\mathbf{1}^{\prime}\right)=\vec{\lambda}$ and hence are already determined. Thus we can assume that $p$ is a finite sequence. Now if $p=p^{\prime} \frown(\dot{\gamma}, \dot{q})$ is a finite sequence, then we can find $p^{\prime \prime} \leq p^{\prime}$ so that $p^{\prime \prime}$ determines the value of $\dot{\gamma}$. Thus, if the proposition is false, then there is a counterexample of the form $p=p^{\prime}(\gamma, \dot{q})$, with $\gamma$ 
determined. Pick such a counterexample with $\gamma=\sup ($ domain $p)$ as small as possible. Then by the same argument there is $p^{\prime \prime} \leq p^{\prime}$ such that $\gamma^{\prime \prime}=\sup \left(\right.$ domain $\left.p^{\prime \prime}\right)$ is determined and $\gamma^{\prime \prime} \leq \sup \left(\right.$ domain $\left.p^{\prime}\right)<\gamma$. But then $p^{\prime \prime}$ is also a counterexample to the proposition, contradicting the minimality of $\gamma$.

This proposition is less useful than might be hoped, due to the fact that it is false if $\leq$ is replaced by $\leq^{*}$.

We now are now ready to give the definitions of the four recipes. For each recipe, we will specify its set of instances together with rules for the direct extension order $q^{\prime} \leq^{*} q$ and the forcing order $p \leq(\lambda, q)$. In addition we define the floor $\lfloor(\lambda, q)\rfloor$.

In the case of $\leq$, the extensions specified by these rules should be regarded as onestep extensions; an arbitrary extension will be obtained from these by transitivity and definition 2.8 .

The recipe $P_{\lambda}$ is the primary means by which ordinals can be added to the domain of a condition $p$. It is also used to specify legal values of $h(\nu)$ in the recipe for $Q_{\lambda}$.

Definition 2.10 (The recipe $P)$. $\quad$ 1. The recipe $P_{\lambda}$ is indicated by the pair $(\lambda, \bar{\lambda})$, with $\bar{\lambda}<\lambda$. We set $\lfloor(\lambda, \bar{\lambda})\rfloor=\bar{\lambda}$; thus any condition $p^{\frown}(\lambda, \bar{\lambda})$ will satisfy $\lceil p\rceil<\bar{\lambda}$.

2. If $\bar{\lambda}<\bar{\lambda}^{\prime}<\lambda$ then $\left(\lambda, \bar{\lambda}^{\prime}\right) \leq *(\lambda, \bar{\lambda})$.

3. In $\mathcal{R}_{\lambda+1}^{\prime}$, the relations $p^{-}\left(\lambda, \bar{\lambda}^{\prime}\right) \leq(\lambda, \bar{\lambda})$ and $p^{-}\left(\lambda, \bar{\lambda}^{\prime}\right) \leq *(\lambda, \bar{\lambda})$ are equivalent, and hold if and only if $\lfloor p\rfloor<\bar{\lambda}, p \Vdash\lceil p\rceil<\bar{\lambda}^{\prime}$, and $p$ only uses instances of the recipe $Q$.

4. In $\mathcal{R}_{\lambda+1}$ and $\mathbf{R}_{\lambda+1}$, the relation $p^{-}\left(\lambda, \bar{\lambda}^{\prime}\right) \leq^{*}(\lambda, \bar{\lambda})$ holds whenever

(a) $\bar{\lambda}<\lfloor p\rfloor$ and $p \Vdash\lceil p\rceil<\bar{\lambda}^{\prime}$.

(b) $p$ is $\bar{\lambda}^{+}$-closed; that is, $p$ involves only instances of $Q$, and of $Q_{\gamma}^{*}$ and $U_{\gamma, 0} \times Q_{\gamma}^{*}$ for $\gamma$ with $o(\gamma) \geq \bar{\lambda}^{+}$.

(c) In the case of $\mathbf{R}_{\lambda+1},\left(p^{-}\left(\lambda, \bar{\lambda}^{\prime}\right), \vec{B}^{\prime}\right) \leq^{*}((\lambda, \bar{\lambda}), \vec{B})$ requires that any instance of $U_{\gamma, 0} \times Q_{\gamma}^{*}$ in $p$ satisfies condition 2.15)(2) below. (This clause, which depends on the sequence $\vec{B}$, is ignored in defining $\leq$ in $\mathcal{R}_{\lambda+1}$.)

5. The relation $p^{\frown}\left(\lambda, \bar{\lambda}^{\prime}\right) \leq(\lambda, \bar{\lambda})$ holds in $\mathbf{R}_{\lambda}$ only if it follows from clause 4 and definition [2.8, that is, if there is $p^{\prime}$ such that $p \leq p^{\prime}$ and $p^{\prime}-\left(\lambda, \bar{\lambda}^{\prime}\right) \leq^{*}(\lambda, \bar{\lambda})$.

Except where otherwise stated, the definitions for $P$ also apply to instances of $Q^{*}$ and $U \times Q^{*}$ representing instances of $P$.

Remark 2.11. The second coordinate $\bar{\lambda}$ of $(\lambda, \bar{\lambda})$ is used to prevent an overlapping situation where $\eta_{0}<\eta_{1}<\gamma_{0}<\gamma_{1}$ and instances of $P_{\eta_{0}}$ and $P_{\eta_{1}}$ are spawned from instances of $U_{\gamma_{0}, 0} \times Q_{\gamma_{0}}^{*}$ and $U_{\gamma_{1}, 0} \times Q_{\gamma_{1}}^{*}$, respectively. This is at best a minor convenience for $\mathcal{R}_{\kappa}^{\prime}$, but is probably necessary for $\mathbf{R}_{\kappa+1}$.

In the case of $\mathbf{R}_{\lambda}$, a non-direct inequality $p^{-}\left(\lambda, \xi^{\prime}\right) \leq(\lambda, \xi)$ can occur in two steps, via an instance of $U_{\gamma, 0} \times Q_{\gamma}^{*}$ :

$$
(\eta, \xi)^{\frown}(\lambda, \gamma) \leq(\gamma, 0, \xi)^{\frown}(\lambda, \gamma) \leq^{*}(\lambda, \xi) .
$$

The effect of the floor $\xi$ of the condition $(\lambda, \xi)$ is to ensure that the effect of having $\gamma \in$ domain $p$ is preserved in the left hand extension above, that is, to ensure that any extension $(\eta, \xi)^{\frown} p^{\prime} \frown\left(\lambda, \gamma^{\prime}\right) \leq(\xi, \xi)^{\frown}(\lambda, \gamma) \leq(\lambda, \xi)$ satisfies $\left\lfloor p^{\prime}\right\rfloor>\gamma$. This is needed for the proof of lemma 4.4 
Definition 2.12 (The recipe $Q$ ). 1 . The recipe $Q_{\lambda}$ is indicated by a triple $(\lambda, A$, $h$ ), where $A \in U(\lambda)$ and $h$ is a function mapping ordinals $\nu \in A$ to $\mathcal{R}_{\nu+1}$-terms $h(\nu)=\dot{p}$ denoting a condition $p \in \mathcal{R}_{\nu, \lambda}^{V[C \cap \nu]}$ such that $\Vdash \dot{p}^{\frown}(\lambda,\lceil\dot{p}\rceil) \leq^{*}(\lambda, \nu)$ in $\mathcal{R}_{\lambda+1}^{\prime}$ or in $\mathcal{R}_{\lambda+1}$ as appropriate.

2. We say that $\left(\lambda, A^{\prime}, h^{\prime}\right) \leq^{*}(\lambda, A, h)$ if $A^{\prime} \subset A$ and $\Vdash h^{\prime}(\nu) \leq^{*} h(\nu)$ for all $\nu \in A^{\prime}$. The measure one sequence $\vec{B}$ in $\mathbf{R}_{\lambda}$ does not come into play in this case.

3. In addition, we have (subject, in the case of $\mathbf{R}_{\lambda+1}$, to clause [2.15)(2) ) the following non-direct extension:

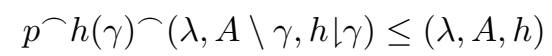

whenever $\gamma \in A, A \cap \gamma \in U(\gamma)$ and $p$ is a condition such that $p \leq(\gamma, A \cap \gamma, h\lceil\gamma)$ and $p$ determines the length and recipes of $h(\gamma)$.

4. If $\beta<o(\lambda)$ then we write $Q_{\lambda, \beta}$ for $Q_{\lambda}^{\text {ult }\left(V, U_{\lambda, \beta}\right)}$. Equivalently, $Q_{\lambda, \beta}$ can be defined in $V$ exactly like $Q_{\lambda}$, except that $\bigcap_{\eta<\beta} U_{\lambda, \eta}$ is used instead of $U(\lambda)=$ $\bigcap_{\eta<o(\lambda)} U_{\lambda, \eta}$

5. We set $\lfloor(\lambda, A, h)\rfloor=\bigcap A$. We will use the same definition for instances of $Q_{\lambda}^{*}$ or $U_{\lambda, 0} \times Q_{\lambda}^{*}$ derived from $Q_{\lambda}$.

Clause 1 implies that $h(\nu)$ is $\nu^{+}$closed.

The definition of $\leq$ in clause 3 essentially asserts that

$$
(\gamma, A \cap \gamma, h\lceil\gamma) \frown h(\gamma) \frown(\lambda, A \backslash \gamma, h \backslash \gamma) \leq(\lambda, A, h),
$$

which, if the function $h$ is ignored, is the basic one-step extension in Prikry-Magidor forcing. However, the left side of (4) may not be a condition since $(\gamma, A \cap \gamma, h\lceil\gamma)$ may not determine, in $\mathbf{R}_{\gamma+1}$ or $\mathcal{R}_{\gamma+1}^{\prime}$, the size of domain $h(\nu)$ or the specific recipes used in this condition.

Proposition 2.13. The ordering $\leq^{*}$ in $Q_{\lambda}$ is diagonally $\lambda$-closed in the following sense: suppose that $\left(\left(\lambda, A_{\xi}, h_{\xi}\right): \xi<\lambda\right)$ is a $\leq^{*}$-descending sequence of conditions. Define $A^{\prime}$ to be the diagonal intersection of the sets $A_{\xi}$, and define $h^{\prime}(\nu)=$ $\bigwedge_{\xi<\nu} h_{\xi}(\nu)$. Then $\left(\lambda, A^{\prime}, h^{\prime}\right)$ is in $Q_{\lambda}$, and $\left(\lambda, A^{\prime} \backslash \xi, h^{\prime}\right) \leq^{*}\left(\lambda, A_{\xi}, h_{\xi}\right)$ for all $\xi<\lambda$.

Note that $\bigwedge_{\xi<\nu} h_{\xi}(\nu)$ must exist, because the condition $h(\nu)$ is required to be $\nu^{+}$-closed. We write $\left(\lambda, A^{\prime}, h^{\prime}\right)=\triangle_{\xi<\lambda}\left(\lambda, A_{\xi}, h_{\xi}\right)$ for this diagonal limit.

Definition 2.14 (The recipe $Q^{*}$ ). 1 . The recipe $Q_{\lambda}^{*}$, which is only defined if $o(\lambda)$ is a nonzero limit ordinal, is indicated by either a triple $(\lambda, 0, \bar{\lambda})$ such that $(\lambda, \bar{\lambda}) \in P_{\lambda}$, or by a quadruple $(\lambda, \beta, A, h)$ such that $0<\beta<\min (o(\lambda), \lambda)$ and $(\lambda, A, h) \in Q_{\lambda, \beta}$.

2. The direct extension $\leq^{*}$ is defined by the following three rules:

(a) $\left(\lambda, 0, \bar{\lambda}^{\prime}\right) \leq^{*}(\lambda, 0, \bar{\lambda})$ if $\bar{\lambda} \leq \bar{\lambda}^{\prime}<\lambda$.

(b) $(\lambda, \beta, A, h) \leq *(\lambda, 0, \bar{\lambda})$ if $\inf (A) \geq \bar{\lambda}$.

(c) $\left(\lambda, \beta^{\prime}, A^{\prime}, h^{\prime}\right) \leq^{*}(\lambda, \beta, A, h)$ if $\beta^{\prime} \geq \beta$ and $\left(\lambda, A_{<\beta}^{\prime}, h^{\prime} \uparrow A_{<\beta}^{\prime}\right) \leq^{*}(\lambda, A, h)$ in $Q_{\lambda, \beta}$, where $A_{<\beta}^{\prime}=\left\{\nu \in A^{\prime}: o(\nu)<\beta\right\}$.

3. There are two additional rules to define the forcing order $\leq$ :

(a) $p^{-}\left(\lambda, 0, \bar{\lambda}^{\prime}\right) \leq(\lambda, 0, \bar{\lambda})$ whenever $p^{\frown}\left(\lambda, \bar{\lambda}^{\prime}\right) \leq(\lambda, \bar{\lambda})$ in $P_{\lambda}$.

(b) $(\gamma, A \cap \gamma, h\lceil\gamma) \frown(\lambda, 0, \gamma) \leq(\lambda, \beta, A, h)$ whenever $o(\gamma)=\beta$ and $(\gamma, A \cap$ $\gamma, h\lceil\gamma) \in Q_{\gamma}$. 
By clauses $2 \mathrm{a}$ and $3 \mathrm{a}$, an instance $(\lambda, 0, \bar{\lambda})$ may be identified with the instance $(\lambda, \bar{\lambda})$ of $P_{\lambda}$. By clause $2 \mathrm{~b}$ the form $(\lambda, 0, \bar{\lambda})$ could be regarded as an instance $(\lambda, 0, \varnothing, \varnothing)$ of the second form of $Q_{\lambda}^{*}$ with $\beta=0$, except that the latter lacks the coordinate $\bar{\lambda}$.

Definition $2.15\left(U_{\lambda, 0} \times Q_{\lambda}^{*}\right)$. $\quad$ 1. This recipe, which is only defined if $o(\lambda)$ is a nonzero limit ordinal, has one of the forms $(\lambda, B, \bar{\lambda})$ or $(\lambda, B, \beta, A, h)$. In either case $B \in \mathcal{P}(D) \cap U_{\lambda, 0}$ and $(\lambda, 0, \bar{\lambda})$ or $(\lambda, \beta, A, h)$ is in $Q_{\lambda}^{*}$.

2. (See definition 2.8) If $\left(p^{\prime}, \vec{B}^{\prime}\right)$ and $(p, \vec{B})$ are conditions in $\mathbf{R}_{\gamma}$, then $\left(p^{\prime}, \vec{B}^{\prime}\right) \leq$ $(p, \vec{B})$ if (i) $p^{\prime} \leq p$ in $\mathcal{R}_{\gamma}$, and (ii) for each ordinal $\gamma \in \operatorname{domain}\left(p^{\prime}\right) \backslash \operatorname{domain}(p)$ such that $p^{\prime}$ has an instance $(\gamma, B, q)$ of $U_{\gamma, 0} \times Q_{\gamma}^{*}$ we have $B \subset B_{\gamma}$.

3. If $\left(\lambda, B^{\prime}, q^{\prime}\right)$ and $(\lambda, B, q)$ are instances of $U_{\lambda, 0} \times Q_{\lambda}^{*}$, then $\left(\lambda, B^{\prime}, q^{\prime}\right) \leq^{*}$ $(\lambda, B, q)$ if $B^{\prime} \subset B$ and $\left(\lambda, q^{\prime}\right) \leq^{*}(\lambda, q)$ as instances of $Q_{\lambda}^{*}$.

4. The forcing order $\leq$ is defined by the following two additional rules:

(a) $p^{\frown}(\lambda, B, 0, \bar{\lambda}) \leq(\lambda, B, q)$ whenever $p^{\frown}(\lambda, 0, \bar{\lambda}) \leq(\lambda, q)$ in $Q_{\lambda}^{*}$.

(b) $(\gamma, \bar{\lambda}) \leq(\lambda, B, 0, \bar{\lambda})$ whenever $\gamma \in B$ and $o(\gamma)=0$.

This is the only recipe on $\lambda$ which does not force $\lambda \in C$. As was pointed out in an earlier remark, it is also the only way in which an instance of a recipe $P_{\gamma}$ (except for $\gamma=\kappa$ in $\mathcal{R}_{\kappa+1}$ ) will ever occur in a condition.

Remark 2.16. The difference between $\mathcal{R}_{\lambda}$ and $\mathbf{R}_{\lambda}$ appears only in Clause 2.15(2), as this is the only place where the sequence $\vec{B}$ is used.

Notice that, in a sense precisely stated in proposition 4.3 below, the sequence $\vec{B}$ in a condition $(p, \vec{B})$ has no essential effect on the appearance of new instances of $U \times Q^{*}$. Instead, its real effect is to restrict the appearance of new instances of $P$. If $\left(p^{\prime}, \vec{B}^{\prime}\right) \leq(p, \vec{B})$ then any instance of $P_{\gamma}$ in $p^{\prime}$ with $\gamma \notin$ domain $p$ must be spawned, via rule $2.15(4 \mathrm{~b})$, by some instance of $U \times Q^{*}$ either in $p$ or in a condition intermediate between $\left(p^{\prime}, \vec{B}^{\prime}\right)$ and $\left.(p, \vec{B})\right)$. It follows that if $\lambda=\inf ($ domain $p \backslash \gamma)$ then either (i) $p$ has an instance $(\lambda, B, q)$ of $U_{\lambda, 0} \times Q_{\lambda}^{*}$, and $\gamma \in B$ or (ii) there is some $\gamma^{\prime} \in \lambda \backslash \gamma$ such that $\gamma \in B_{\gamma^{\prime}}$ and $\operatorname{cf}\left(o\left(\gamma^{\prime}\right)\right) \geq\lceil p\rceil\lceil\gamma$.

\section{The PRoof of Theorem 1.5}

Let $C$ be a $\mathcal{R}_{\kappa}^{\prime}$-generic subset of $\kappa$ over $V$. The initial condition

$$
\mathbf{1}^{\prime}=\left(\left(\lambda_{n}, 0, \lambda_{n-1}\right): n<\omega\right)
$$

of $\mathcal{R}_{\kappa}^{\prime}$ consists of $\omega$-many instances of the recipe $U_{\lambda_{n}, 0} \times Q_{\lambda_{n}}^{*}$, each of which will spawn an instance of $P_{\kappa_{n}}$ for some $\kappa_{n} \in\left(\lambda_{n-1}, \lambda_{n}\right)$. Each of these instances of $P_{\kappa_{n}}$ generates a closed and unbounded subset $C \cap\left(\lambda_{n-1}, \kappa_{n}\right)$ of $E \cap \kappa_{n}$, so we only need to prove that no cardinals of $V$ are collapsed in $V[C]$ and that the ordinals $\kappa_{n}$ remain inaccessible in $V[C]$.

Most of the arguments in this section will be reused in section 4, where we will describe how to modify the proof to prove the analogous results for the forcing $\mathbf{R}_{\kappa+1}$ used for the main theorem.

For convenience, we will use $P_{\lambda}^{\prime}$ to mean the partial ordering with the same universe as $\mathcal{R}_{\lambda}^{\prime}$ and with the order defined by $p^{\prime} \leq_{P} p$ if $p^{\prime} \frown\left(\lambda,\left\lceil p^{\prime}\right\rceil\right) \leq p \frown(\lambda,\lceil p\rceil)$ in $\mathcal{R}_{\lambda}^{\prime}$. Thus, regarded as a forcing notion, $\left(P_{\lambda}^{\prime}, \leq_{P}\right)$ allows end extensions while $\mathcal{R}_{\lambda}^{\prime}$ does not.

The following lemma is our adaptation of Gitik's proof that the preliminary forcing of [2] makes $P_{\lambda}[E]$ distributive. 
Lemma 3.1 (Distributivity of $\left.P_{\lambda}^{\prime}\right)$. Suppose that $\bar{\lambda}<\lambda<\kappa, \lambda \in D$ and $\tau<\lambda$. Then for any sequence $\overrightarrow{\mathcal{D}}=\left(\mathcal{D}_{\xi}: \xi<\tau\right)$ of dense open subsets of $\left(P_{\lambda}^{\prime}, \leq_{P}\right)$ there is a condition $p \in \mathcal{R}_{\lambda}^{\prime}$, with $\lfloor p\rfloor \geq \bar{\lambda}$, such that $\mathcal{D}_{\xi}$ is dense in $\mathcal{R}^{\prime} / p$ for all $\xi<\tau$.

Hence, if $\dot{H}$ is a name for the generic subset of $P_{\lambda}^{\prime}$ then $p$ forces that $\dot{H} \cap \mathcal{R}_{\lceil p\rceil+1} \cap$ $\mathcal{D}_{\xi} \neq \varnothing$ for all $\xi<\tau$.

Proof. Let $S$ be the set of ordinals $\gamma<\lambda$ such that there is a set $X_{\gamma} \prec\left(\mathrm{H}_{\lambda^{+}}, \in\right)$ with $\left\{\lambda, \bar{\lambda},\left(\mathcal{D}_{\xi}: \xi<\tau\right)\right\} \subset X_{\gamma}$ and $\gamma=X_{\gamma} \cap \lambda$. Then $S$ contains a closed and unbounded subset of $\lambda$, so since $\lambda \in D$ there is $\gamma \in S$ with $o(\gamma)=\tau$. Let $A=\{\nu \in$ $\gamma \backslash \bar{\lambda}: o(\nu)<\tau\}$. We will define a function $h$ so that the condition $p=(\gamma, A, h)$ has the required property.

Let $\nu$ be an arbitrary member of $A$. Since $\gamma$ is in $S, \mathcal{D}_{o(\nu)}$ is dense in $\mathcal{R}_{\gamma}^{\prime}$. We can factor $\mathcal{R}_{\gamma}^{\prime}=\mathcal{R}_{\nu+1}^{\prime} * \dot{\mathcal{R}}_{\nu+1, \gamma}^{\prime}$, and hence there is a $\mathcal{R}_{\nu+1}^{\prime}$ term $\dot{p}_{1}$ denoting a member of $\mathcal{R}_{\nu+1, \gamma}^{\prime}$ such that $\Vdash_{\mathcal{R}_{\nu+1}^{\prime}} \exists p_{0} \in \mathfrak{G}(C \cap \nu) p_{0} \frown \dot{p}_{1} \in \mathcal{D}_{o(\nu)}$. Set $h(\nu)=\dot{p}_{1}$.

To see that $p=(\gamma, A, h)$ has the required property, fix an arbitrary ordinal $\xi<\tau$ and condition $p^{\prime}=p_{0}^{\prime} \frown\left(\gamma, A^{\prime}, h^{\prime}\right) \leq(\gamma, A, h)$. We will find a condition $p^{\prime \prime} \leq p$ in $\mathcal{D}_{\xi}$. Pick any ordinal $\nu \in A^{\prime} \backslash\left\lceil p_{0}^{\prime}\right\rceil$ with $o(\nu)=\xi$ and $\left(\nu, A^{\prime} \cap \nu, h^{\prime} \uparrow \nu\right) \in Q_{\nu}$. By the choice of $h(\nu)$ there is $p^{\prime \prime} \leq p_{0}^{\prime} \frown\left(\nu, A^{\prime} \cap \nu, h^{\prime}\lceil\nu)\right.$ such that $p^{\prime \prime} \frown h(\nu) \in \mathcal{D}_{\xi}$. Then $p^{\prime \prime} \frown h(\nu) \frown\left(\gamma, A^{\prime}, h^{\prime}\right) \leq p^{\prime}$, and $p^{\prime \prime} \frown h(\nu) \frown\left(\gamma, A^{\prime}, h^{\prime}\right) \in \mathcal{D}_{\xi}$ since $\mathcal{D}_{\xi}$ is open.

Corollary 3.2. If $\lambda \in D$ and $C \subset \lambda$ is $V$-generic for $\mathcal{R}_{\lambda+1}^{\prime}$, and $f: \tau \rightarrow V$ is a function in $V[C]$ with $\tau<\lambda$, then there is $\gamma<\lambda$ such that $f \in V[C \cap \gamma]$.

Proof. Apply lemma 3.1 with $\mathcal{D}_{\xi}=\{p\lceil\lambda: \exists x \exists \bar{\lambda} p \Vdash \dot{f}(\xi)=\check{x}\}$. Then the condition $p \frown(\lambda, \gamma) \leq(\lambda, \bar{\lambda})$ from lemma 3.1 with $\gamma=\lceil p\rceil$, forces that $\dot{f} \in V[C \cap \gamma]$.

Lemma 3.3 (Prikry property for $\mathcal{R}_{\kappa}^{\prime}$ ). If $\tau \leq \lambda \leq \kappa$ then $\mathcal{R}_{\tau, \lambda}^{\prime}$ has the $\tau^{+}$-Prikry property.

Proof. We assume as an induction hypothesis that $\mathcal{R}_{\eta, \lambda^{\prime}}^{\prime}$ has the $\eta^{+}$-Prikry property for all $\eta<\lambda^{\prime}<\lambda$. The proof that $\mathcal{R}_{\tau, \lambda}^{\prime}$ has the $\tau^{+}$-Prikry property falls into several cases.

Case 3.3.1. $\lambda=\lambda^{\prime}+1$.

We can assume that $\lambda^{\prime} \in E$, since otherwise $\mathcal{R}_{\lambda}^{\prime}=\mathcal{R}_{\lambda^{\prime}}^{\prime}$, which has the $\tau$-Prikry property by the induction hypothesis.

We claim that it suffices to show that any condition of the form $\left(\lambda^{\prime}, q\right)$ has the $\tau$-Prikry property. To see this, note that an arbitrary condition in $\mathcal{R}_{\tau, \lambda}^{\prime}$ has the form $p^{\frown}(\dot{\gamma}, \dot{q})$, where $\dot{q}$ is $C_{p^{-}}$-term for a recipe on some $\gamma \leq \lambda^{\prime}$. Since $\mathcal{R}_{\tau, \lambda^{\prime}}^{\prime}$ has the $\tau$-Prikry property by the induction hypothesis, we can assume that $p \|_{\mathcal{R}_{\tau, \lambda^{\prime}}^{\prime}} \dot{\gamma}=\lambda^{\prime}$. If $p \Vdash_{\mathcal{R}_{\tau, \lambda^{\prime}}} \dot{\gamma}<\lambda^{\prime}$ then $p^{-} \dot{q} \in \mathcal{R}_{\tau, \lambda^{\prime}}^{\prime}$, which has the $\tau$-Prikry property, so we can assume that $p \Vdash_{\mathcal{R}_{\tau, \lambda^{\prime}}^{\prime}} \dot{\gamma}=\lambda^{\prime}$.

We will work in $V\left[C_{p}\right]$ and show there that there is a condition $p^{\prime} \leq^{*}\left(\lambda^{\prime}, q\right)$ which decides each sentence $\sigma_{\xi}$. To see that this suffices, let $\dot{p}^{\prime}$ be a $C_{p}$-term for such a condition, so that $p \Vdash_{\mathcal{R}_{\tau, \lambda}^{\prime}} \forall \xi<\tau \exists \bar{p} \in \mathfrak{G}\left(C_{p}\right) \bar{p}^{\frown} \dot{p}^{\prime} \|_{\mathcal{R}_{\tau, \lambda}} \sigma_{\xi}$. By the induction hypothesis there is a condition $p^{\prime \prime} \leq^{*} p$ which decides the sentence $\exists \bar{p} \in \mathfrak{G}\left(C_{p}\right) \bar{p} \frown \dot{p}^{\prime} \Vdash \sigma_{\xi}$ for each $\xi<\tau$, but then $p^{\prime \prime} \dot{p}^{\prime} \|_{\mathcal{R}_{\tau, \lambda}} \sigma_{\xi}$ for each $\xi<\tau$.

We use four subcases to show that $\left(\lambda^{\prime}, q\right)$ has the $\tau$-Prikry property, one for each of the recipes $P_{\lambda^{\prime}}, Q_{\lambda^{\prime}}, Q_{\lambda^{\prime}}^{*}$ and $U_{\lambda^{\prime}, 0} \times Q_{\lambda^{\prime}}^{*}$. The recipe $Q_{\lambda^{\prime}}^{*}$ is included, even 
though it is not used in $\mathcal{R}_{\tau, \kappa}^{\prime}$, since it is used in the proof for the case $U_{\lambda^{\prime}, 0} \times Q_{\lambda^{\prime}}^{*}$ and also will be used to define the measure on $\kappa$ in the proof of theorem 1.1

Subcase 3.3.1a. $\left(\lambda^{\prime}, q\right)=\left(\lambda^{\prime}, \bar{\lambda}^{\prime}\right)$, an instance of $P_{\lambda^{\prime}}$.

If we set $\mathcal{D}_{\xi}=\left\{p \in P_{\lambda^{\prime}}: \exists \eta p^{\frown}\left(\lambda^{\prime}, \eta\right) \| \sigma_{\xi}\right\}$, then $\mathcal{D}_{\xi}$ is dense and open in $\left(P_{\tau, \lambda^{\prime}}, \leq_{P}\right)$. It follows by lemma 3.1 that there is $p \in \mathcal{R}_{\tau, \lambda^{\prime}}^{\prime}$ such that $p \Vdash \forall \xi<$ $\tau \exists \bar{p} \in \mathfrak{G}\left(C_{p}\right) \bar{p} \in \mathcal{D}_{\xi}$. Since $\lambda^{\prime}$ is regular and $\left|\tau \times \mathcal{R}^{\prime} / p^{\prime}\right|<\lambda^{\prime}$, it follows that there is $\eta<\lambda^{\prime}$ so that $p \Vdash \forall \xi<\tau \exists \bar{p} \in \mathfrak{G}\left(C_{p}\right) \bar{p} \frown\left(\lambda^{\prime}, \eta\right) \| \sigma_{\xi}$. Since $\mathcal{R}_{\tau, \lambda^{\prime}}^{\prime}$ has the $\tau$-Prikry property there is $p^{\prime} \leq^{*} p$ such that $p^{\prime} \| \exists \bar{p} \in \mathfrak{G}\left(C_{p}\right) \bar{p} \frown\left(\lambda^{\prime}, \eta\right) \Vdash \sigma_{\xi}$ for each $\xi<\kappa$, but then $p^{\prime}-\left(\lambda^{\prime}, \eta\right) \| \sigma_{\xi}$ for each $\xi<\kappa$.

Subcase 3.3.1b. $\left(\lambda^{\prime}, q\right)$ is an instance of the recipe $Q_{\lambda^{\prime}}$.

The proof of this subcase will take up more than half of the full length of the proof of lemma 3.3. Since $Q_{\lambda^{\prime}}$ is $<\lambda^{\prime}$-closed, it is sufficient to show that $Q_{\lambda^{\prime}}$ has the Prikry property, that is, that for any single sentence $\sigma$ and instance $\left(\lambda^{\prime}, A, h\right)$ of $Q_{\lambda^{\prime}}$ there is $\left(\lambda^{\prime}, A^{\prime}, h^{\prime}\right) \leq^{*}\left(\lambda^{\prime}, A, h\right)$ which decides $\sigma$. Define, for a condition $(\lambda, A, h)$ and an ordinal $\xi \in A$ such that $(\xi, A \cap \xi, h \uparrow \xi) \in Q_{\xi}$, the condition

$$
r(A, h, \xi)=(\xi, A \sqcap \xi, h\lceil\xi) \frown h(\xi)
$$

where

$$
A \sqcap \xi=\{\nu \in A \cap \xi: o(\nu)<o(\xi)\} .
$$

Note that $r(A, h, \xi)$, for an arbitrary condition $(\lambda, A, h)$, is in general a term for a member of $\mathcal{R}_{\xi, \lambda}^{\prime}$, but not necessarily a condition.

Claim. For any condition $\left(\lambda^{\prime}, A, h\right)$ there is $\left(\lambda^{\prime}, A^{\prime}, h^{\prime}\right) \leq^{*}\left(\lambda^{\prime}, A, h\right)$ so that $r\left(A^{\prime}, h^{\prime}, \xi\right)$ is a condition for each $\xi \in A^{\prime}$.

Proof. Fix some $\beta<o\left(\lambda^{\prime}\right)$. We will find $\left(\lambda^{\prime}, A^{\prime}, h^{\prime}\right) \leq^{*}\left(\lambda^{\prime}, A, h\right)$ so that $r\left(A^{\prime}, h^{\prime}, \xi\right)$ is a condition for each $\xi \in A^{\prime}$ with $o(\xi)=\beta$. We can then find the condition required by the claim as the limit of a descending sequence of length $o\left(\lambda^{\prime}\right)$.

For each $\xi \in A$ with $o(\xi)=\beta$, use the induction hypothesis to pick $\tilde{A}_{\beta}$ and $h_{\beta}$ so that $\left(\xi, \tilde{A}_{\beta}, \tilde{h}_{\beta}\right) \leq^{*}\left(\xi, A \sqcap \xi, h\lceil\xi)\right.$ so that $\left(\xi, \tilde{A}_{\beta}, \tilde{h}_{\beta}\right)$ decides the size of $h(\xi)$ and the recipes appearing in it. Then there is a unique $\tilde{A}$ and $\tilde{h}$ so that $\bar{A}=\left\{\xi \in A: o(\xi)=\beta\right.$ and $\tilde{A}_{\xi}=\tilde{A} \cap \xi$ and $\left.\tilde{h}_{\xi}=\tilde{h} \mid \xi\right\} \in U_{\lambda^{\prime}, \beta}$.

Let $A^{\prime}$ be the set of $\nu \in A$ such that if $o(\nu)<\beta$, then $\nu \in \tilde{A}$ and if $o(\nu)=\beta$, then $\nu \in \bar{A}$. Set $h^{\prime}(\nu)=\tilde{h}(\nu)$ if $o(\nu)<\beta$ and $h^{\prime}(\nu)=h(\nu)$ otherwise.

Define $p^{\prime} \leq^{\prime} p$ if $p^{\prime} \leq p$ and $\min \left(\right.$ domain $\left.p^{\prime}\right)=\min (\operatorname{domain} p)$. Thus $p \leq^{\prime} r(A, h, \xi)$ if and only if $p$ has the form $(\xi, \bar{A}, \bar{h})^{\frown} p_{1}$ where $(\xi, \bar{A}, \bar{h}) \leq^{*}(\xi, A \sqcap \xi, h\lceil\xi)$ and $p_{1} \leq h(\xi)$.

Claim. For all $\beta<o\left(\lambda^{\prime}\right)$, all $p_{0} \in \mathcal{R}_{\tau, \lambda}$, and all instances $\left(\lambda^{\prime}, A, h\right)$ of $Q_{\lambda^{\prime}}$, there is a condition $\left(\lambda^{\prime}, A^{\prime}, h^{\prime}\right) \leq^{*}\left(\lambda^{\prime}, A, h\right)$ so that if $\bar{\sigma}$ is one of $\sigma$ or $\neg \sigma$ and if $\nu \in$ $A^{\prime}, p_{1} \leq^{\prime} r\left(A^{\prime}, h^{\prime}, \nu\right)$ and $\left(\lambda^{\prime}, \bar{A}, \bar{h}\right) \leq^{*}\left(\lambda^{\prime}, A^{\prime}, h^{\prime}\right)$ are such that $o(\nu)=\beta$ and $p_{0} \frown p_{1} \frown\left(\lambda^{\prime}, \bar{A}, \bar{h}\right) \Vdash \bar{\sigma}$, then

$$
\forall \nu^{\prime} \in A^{\prime} \backslash\left\lceil p_{0}\right\rceil \quad\left(o\left(\nu^{\prime}\right)=\beta \Longrightarrow p_{0} \frown r\left(A^{\prime}, h^{\prime}, \nu^{\prime}\right) \frown\left(\lambda^{\prime}, A^{\prime}, h^{\prime}\right) \Vdash \bar{\sigma}\right) .
$$


Proof. We will define, by recursion on $\nu$, a $\leq^{*}$-decreasing sequence of conditions $\left(\lambda^{\prime}, A_{\nu}, h_{\nu}\right)$ for $\nu<\lambda^{\prime}$. In addition, we will define for all $\nu<\lambda^{\prime}$ such that

$$
\nu \in \bigcap_{\nu^{\prime}<\nu} A_{\nu} \quad \text { and } \underset{\nu^{\prime}<\nu}{\triangle}\left(\nu, A_{\nu^{\prime}} \sqcap \nu, h_{\nu^{\prime}} \mid \nu\right) \in Q_{\nu},
$$

conditions $\left(\nu, \tilde{A}_{\nu}, \tilde{h}_{\nu}\right) \leq^{*} \triangle_{\nu^{\prime}<\nu}\left(\nu, A_{\nu^{\prime}} \sqcap \nu, h_{\nu^{\prime}}\lceil\nu)\right.$ and $\tilde{p}_{\nu} \leq^{*} h_{\nu}(\nu)$ so that one of the following two cases hold:

$$
\begin{gathered}
p_{0} \frown\left(\nu, \tilde{A}_{\nu}, \tilde{h}_{\nu}\right) \frown \tilde{p}_{\nu} \frown\left(\lambda^{\prime}, A_{\nu+1}, h_{\nu+1}\right) \| \sigma \\
\forall \bar{p} \leq\left(\nu, \tilde{A}_{\nu}, \tilde{h}_{\nu}\right) \frown \tilde{p}_{\nu} \forall\left(\lambda^{\prime}, \bar{A}, \bar{h}\right) \leq^{*}\left(\lambda^{\prime}, A_{\nu+1}, h_{\nu+1}\right) \quad \bar{p}_{0} \frown \bar{p} \frown\left(\lambda^{\prime}, \bar{A}, \bar{h}\right) \nVdash \sigma .
\end{gathered}
$$

Set $\left(\lambda^{\prime}, A_{0}, h_{0}\right)=\left(\lambda^{\prime}, A, h\right)$, and if $\nu$ is a limit ordinal then set $\left(\lambda^{\prime}, A_{\nu}, h_{\nu}\right)=$ $\bigwedge_{\nu^{\prime}<\nu}\left(\lambda^{\prime}, A_{\nu^{\prime}}, h_{\nu^{\prime}}\right)$. Now suppose that $\left(\lambda^{\prime}, A_{\nu}, h_{\nu}\right)$ is defined. If (6) does not hold then set $\left(\lambda^{\prime}, A_{\nu+1}, h_{\nu+1}\right)=\left(\lambda^{\prime}, A_{\nu}, h_{\nu}\right)$; otherwise define $\left(\lambda^{\prime}, A_{\nu+1}, h_{\nu+1}\right) \leq^{*}$ $\left(\lambda^{\prime}, A_{\nu}, h_{\nu}\right)$ so that

$$
p_{0} \frown \bar{p} \frown\left(\lambda^{\prime}, A_{\nu+1}, h_{\nu+1}\right) \| \sigma
$$

for every $\bar{p} \leq \triangle_{\nu^{\prime}<\nu}\left(\nu, A_{\nu^{\prime}}, h_{\nu^{\prime}}\right) \frown h_{\nu}(\nu)$ for which this is possible. This can be done by specifying $\left(\lambda^{\prime}, A_{\nu+1}, h_{\nu+1}\right)$ to be either a $\mathcal{R}_{\nu+1}$-term for a member of $Q_{\lambda^{\prime}}$ or the infinum of a $\leq{ }^{*}$-descending sequence in $Q_{\lambda^{\prime}}$.

Now use the Prikry property of $\mathcal{R}_{\lambda^{\prime}}$ to find

$$
\left(\nu, \tilde{A}_{\nu}, \tilde{h}_{\nu}\right) \tilde{p}_{\nu} \leq^{*} \underset{\nu^{\prime}<\nu}{\triangle}\left(\nu, \tilde{A}_{\nu^{\prime}} \sqcap \nu, \tilde{h}_{\nu}\lceil\nu) \frown h_{\nu}(\nu)\right.
$$

which decides whether there is a condition $\bar{p} \in \mathfrak{G}\left(C_{\tilde{p}}\right)$ such that 8 holds and, if so, which way $\sigma$ is decided.

This completes the definition of the conditions $\left(\nu, \tilde{A}_{\nu}, \tilde{h}_{\nu}\right) \frown \tilde{p}_{\nu} \frown\left(\lambda^{\prime}, A_{\nu}, h_{\nu}\right)$. Set $\left(\lambda^{\prime}, A_{\lambda^{\prime}}, h_{\lambda^{\prime}}\right)=\triangle_{\nu<\lambda^{\prime}}\left(\lambda^{\prime}, A_{\nu}, h_{\nu}\right)$ and let $X \in U_{\lambda, \beta}$ be a subset of $A_{\lambda^{\prime}}$ such the members $\nu$ of $X$ agree on which of (7a) or (7b) is true and, if (7a) holds, whether $\sigma$ or $\neg \sigma$ is forced. Then there is a unique $\left(\lambda^{\prime}, \tilde{A}, \tilde{h}\right) \in Q_{\lambda^{\prime}, \beta}$ so that

$$
X^{\prime}=\left\{\nu \in X: \tilde{A}_{\nu}=\tilde{A} \cap \nu \text { and } \tilde{h}_{\nu}=\tilde{h}\lceil\nu\} \in U_{\lambda, \beta} .\right.
$$

Finally define $h^{\prime}$ by

$$
h^{\prime}(\nu)= \begin{cases}\tilde{h}(\nu) & \text { if } o(\nu)<\beta, \\ \tilde{p}_{\nu} & \text { if } o(\nu)=\beta, \\ h_{\lambda^{\prime}}(\nu) & \text { if } o(\nu)>\beta,\end{cases}
$$

and let $A^{\prime}$ be the set of $\nu \in A_{\lambda^{\prime}}$ such that $\nu \in \tilde{A}$ if $o(\nu)<\beta$ and $\nu \in X^{\prime}$ if $o(\nu)=\beta$.

If $\nu, p_{1}$, and $\left(\lambda^{\prime}, \bar{A}, \bar{h}\right)$ satisfy the hypothesis of the claim, then

$$
p_{1} \leq\left(\nu, A^{\prime} \sqcap \nu, h^{\prime}\lceil\nu) \frown h^{\prime}(\nu) \leq^{*}\left(\nu, \tilde{A}_{\nu}, \tilde{h}_{\nu}\right)^{\frown} \tilde{p}_{\nu}\right.
$$

and

$$
\left(\lambda^{\prime}, \bar{A}, \bar{h}\right) \leq^{*}\left(\lambda^{\prime}, A^{\prime} \backslash \nu+1, h^{\prime}\lfloor\nu) \leq^{*}\left(\lambda^{\prime}, A_{\nu+1}, h_{\nu+1}\right),\right.
$$

so the conclusion of the claim follows.

By applying the claim to each $\beta<o\left(\lambda^{\prime}\right)$ in turn, we can find a descending sequence of conditions $\left(\lambda^{\prime}, A_{\beta}^{\prime}, h_{\beta}^{\prime}\right)$ so that the single condition $\bigwedge_{\beta}\left(\lambda^{\prime}, A_{\beta}^{\prime}, h_{\beta}^{\prime}\right)$ satisfies the claim for all $\beta<\lambda$. Similarly, we can take a diagonal infinum over all $p_{0} \in \mathcal{R}_{\tau, \lambda^{\prime}}^{\prime}$ 
to find a single condition $\left(\lambda^{\prime}, A^{\prime}, h^{\prime}\right)$ which satisfies the claim for all $\beta<o\left(\lambda^{\prime}\right)$ and all $p_{0} \in \mathcal{R}_{\tau, \lambda^{\prime}}^{\prime}$.

Fix such a condition $\left(\lambda^{\prime}, A^{\prime}, h^{\prime}\right)$ and write $r(\nu)=r\left(A^{\prime}, h^{\prime}, \nu\right)$. Then the last paragraph says that, whenever $\nu \in A$ and

$$
p^{*}=p_{0} \frown p_{1} \frown\left(\lambda^{\prime}, A^{\prime \prime}, h^{\prime \prime}\right) \leq p_{0} \frown r(\nu) \frown\left(\lambda^{\prime}, A^{\prime}, h^{\prime}\right)
$$

is a condition such that $p^{*} \Vdash \sigma$, then $p_{0} \frown r(\nu) \frown\left(\lambda^{\prime}, A^{\prime}, h^{\prime}\right) \Vdash \sigma$ and in fact $p_{0} \frown r\left(\nu^{\prime}\right)^{\frown}$ $\left(\lambda^{\prime}, A^{\prime}, h^{\prime}\right) \Vdash \sigma$ for each $\nu^{\prime} \in A^{\prime} \backslash\left\lceil p_{0}\right\rceil$ with $o\left(\nu^{\prime}\right)=o(\nu)$. Furthermore, the same is true of $\neg \sigma$ in place of $\sigma$.

In order to complete the proof for subcase $3.3 .1 \mathrm{~b}$ we need to show that the condition $\left(\lambda^{\prime}, A^{\prime}, h^{\prime}\right)$ decides the sentence $\sigma$. We will do so by showing that if any condition $p \leq\left(\lambda^{\prime}, A^{\prime}, h^{\prime}\right)$ forces $\sigma$, then $\left(\lambda^{\prime}, A^{\prime}, h^{\prime}\right)$ already forces $\sigma$. The same argument works for $\neg \sigma$, and it follows that $\left(\lambda^{\prime}, A^{\prime}, h^{\prime}\right) \| \sigma$.

Notice that any condition $p \leq\left(\lambda^{\prime}, A^{\prime}, h^{\prime}\right)$ can be written in the form

$$
\begin{aligned}
p & =p_{0} \frown p_{1} \frown \ldots \frown p_{k-1} \frown\left(\lambda^{\prime}, \bar{A}, \bar{h}\right) \\
& \leq r\left(\gamma_{0}\right) \frown r\left(\gamma_{1}\right) \frown \ldots \frown r\left(\gamma_{k-1}\right) \frown\left(\lambda^{\prime}, A^{\prime}, h^{\prime}\right),
\end{aligned}
$$

where $\gamma_{0}<\gamma_{1}<\cdots<\gamma_{k-1}<\lambda^{\prime}$ and $p_{i} \leq^{\prime} r\left(\gamma_{i}\right)$ for each $i<k$. To see this, note that by definition 2.12 any condition $p \leq\left(\lambda^{\prime}, A^{\prime}, h^{\prime}\right)$ must satisfy

$$
\begin{aligned}
p & =p_{0}^{\prime} \frown p_{1}^{\prime} \frown\left(\lambda^{\prime}, \bar{A}, \bar{h}\right) \leq p_{0}^{\prime} \frown r(\gamma) \frown\left(\lambda^{\prime}, A^{\prime}, h^{\prime}\right) \\
& \leq p_{0}^{\prime} \frown\left(\lambda^{\prime}, A^{\prime}, h^{\prime}\right) \leq\left(\lambda^{\prime}, A^{\prime}, h^{\prime}\right)
\end{aligned}
$$

for some conditions $p_{0}^{\prime}$ and $p_{1}^{\prime}$ and some ordinal $\gamma<\lambda^{\prime}$ with $p_{1}^{\prime} \leq r(\gamma)$. We can use the induction hypothesis to represent $p_{0}^{\prime} \frown\left(\lambda^{\prime}, A^{\prime}, h^{\prime}\right) \leq\left(\lambda^{\prime}, A^{\prime}, h^{\prime}\right)$ and $p_{1}^{\prime}\left\lceil\gamma+1 \leq\left(\gamma, A^{\prime} \cap \gamma, h^{\prime}\lceil\gamma)\right.\right.$ in the form of (9)). Now, since $r\left(A^{\prime} \cap \gamma, h^{\prime}\left\lceil\gamma, \gamma^{\prime}\right)=\right.$ $r\left(A^{\prime}, h^{\prime}, \gamma^{\prime}\right)$ for any $\gamma^{\prime} \in A^{\prime} \cap \gamma$, these representations of $p_{0}^{\prime} \frown\left(\lambda^{\prime}, \bar{A}, \bar{h}\right) \leq\left(\lambda^{\prime}, A^{\prime}, h^{\prime}\right)$ and $p_{1}^{\prime}-\left(\lambda^{\prime}, \bar{A}, \bar{h}\right) \leq\left(\lambda^{\prime}, A^{\prime}, h^{\prime}\right)$ can be combined to represent $p^{\prime} \leq\left(\lambda^{\prime}, \bar{A}^{\prime}, h^{\prime}\right)$ in the form (9).

Now suppose that there is a condition below $\left(\lambda^{\prime}, A^{\prime}, h^{\prime}\right)$ which forces $\sigma$, and let $p \leq\left(\lambda^{\prime}, A^{\prime}, h^{\prime}\right)$ be chosen with $\left\lceil p\left\lceil\lambda^{\prime}\right\rceil\right.$ as small as possible so that $p \Vdash \sigma$. By (9), $p$ can be written as $p=p_{0} \frown p_{1} \frown\left(\lambda^{\prime}, \bar{A}, \bar{h}\right)$, where $p_{1} \leq^{\prime} r(\nu)$ for some $\nu<\lambda^{\prime}$. Thus, setting $\beta=o(\nu), p_{0} \frown r\left(\nu^{\prime}\right) \frown\left(\lambda^{\prime}, A^{\prime}, h^{\prime}\right) \Vdash \sigma$ for all $\nu^{\prime} \in A^{\prime} \backslash\left\lceil p_{0}\right\rceil+1$ with $o\left(\nu^{\prime}\right)=\beta$. We will show, under these assumptions, that $\left\{p^{\prime} \in \mathcal{R}_{\tau, \lambda^{\prime}+1}^{\prime}: p^{\prime} \Vdash \sigma\right\}$ is dense below $p_{0} \frown\left(\lambda^{\prime}, A^{\prime}, h^{\prime}\right)$. This will imply $p_{0} \frown\left(\lambda^{\prime}, A^{\prime}, h^{\prime}\right) \Vdash \sigma$, which contradicts the choice of $p$ and hence completes the proof of subcase $3.3 .1 \mathrm{~b}$ for $Q_{\lambda^{\prime}}$.

Let $p^{\prime} \leq p_{0} \frown\left(\lambda^{\prime}, A^{\prime}, h^{\prime}\right)$ be arbitrary. Then, using (9),

$$
p^{\prime}=p_{0}^{\prime} \frown p_{1}^{\prime} \frown \ldots \frown p_{k-1}^{\prime} \frown\left(\lambda^{\prime}, \bar{A}, \bar{h}\right) \leq p_{0} \frown r\left(\gamma_{1}^{\prime}\right) \frown \ldots \frown r\left(\gamma_{k-1}^{\prime}\right) \frown\left(\lambda^{\prime}, A^{\prime}, h^{\prime}\right)
$$

where $p_{0}^{\prime} \leq p_{0}$ and $p_{i}^{\prime} \leq^{\prime} r\left(\gamma_{i}^{\prime}\right)$ for $0<i<k$. We can assume that $o\left(\gamma_{i}^{\prime}\right) \geq \beta$ for some $0<i<k$, if necessary taking a further extension to add $r\left(\gamma_{k}\right)$ for some $\gamma_{k} \in A^{\prime} \backslash \gamma_{k-1}+1$ with $o\left(\gamma_{k}\right)=\beta$. Let $i_{0}$ be the least such $i$. We can further assume that $o\left(\gamma_{i_{0}}\right)=\beta$, since if $o\left(\gamma_{i_{0}}\right)>\beta$ then we could replace $p_{i_{0}}^{\prime}$ with $r(\gamma) \frown p_{i_{0}}^{\prime}$ for some $\gamma \in A^{\prime} \cap\left(\gamma_{i_{0}-1}, \gamma_{i_{0}}\right)$ such that $o(\gamma)=\beta$. Then

$$
\begin{aligned}
p^{\prime} & \leq p_{0} \frown r\left(\gamma_{1}^{\prime}\right) \frown \ldots \frown r\left(\gamma_{k-1}^{\prime}\right) \frown\left(\lambda^{\prime}, A^{\prime}, h^{\prime}\right) \\
& \leq p_{0} \frown r\left(\gamma_{1}^{\prime}\right) \frown \ldots \frown r\left(\gamma_{i_{0}}^{\prime}\right) \frown\left(\lambda^{\prime}, A^{\prime}, h^{\prime}\right) \\
& \leq p_{0} \frown r\left(\gamma_{i_{0}}^{\prime}\right) \Vdash \sigma
\end{aligned}
$$

and hence $p^{\prime} \Vdash \sigma$. 
Subcase 3.3.1c. $\left(\lambda^{\prime}, q\right)$ is one of $\left(\lambda^{\prime}, 0, \bar{\lambda}\right)$ or $(\lambda, \beta, A, h)$, an instance of $Q_{\lambda^{\prime}}^{*}$.

If $\left(\lambda^{\prime}, q\right)=\left(\lambda^{\prime}, 0, \bar{\lambda}\right)$ then set $\beta=0, A=h=\varnothing$ and $X=\left\{\nu \in \lambda^{\prime} \backslash \bar{\lambda}: o(\nu)=0\right\}$. Otherwise let $X$ be the set of $\nu \in \lambda^{\prime} \backslash \bar{\lambda}$ such that $o(\nu)=\beta$ and $\left(\nu, A \cap \nu, h\lceil\nu) \in Q_{\nu}\right.$. In either case $X \in U_{\lambda, \beta}$.

For each $\nu \in X$ use subcase 3.3.1a to pick a $\mathcal{R}_{\tau, \nu+1}$-term $\dot{p}_{\nu}$ denoting a member of $\mathcal{R}_{\nu, \lambda}^{\prime}$ such that $\Vdash_{\mathcal{R}_{\nu}} \forall \xi<\tau \dot{p}_{\nu} \frown\left(\lambda^{\prime}, 0, \eta\right) \|_{\mathcal{R}_{\nu, \lambda}^{\prime}} \sigma_{\xi}$ for some $\eta<\lambda^{\prime}$. By extending $\dot{p}_{\nu}$, if necessary, we can assume that $\eta=\left\lceil\dot{p}_{\nu}\right\rceil$.

Now use the $\tau$-Prikry property of $\mathcal{R}_{\tau, \nu+1}$ to find a condition $\left(\nu, \bar{A}_{\nu}, \bar{h}_{\nu}\right) \leq *$ $(\nu, A, h)$ in $Q_{\nu}$ so that

$$
\forall \xi<\tau\left(\nu, \bar{A}_{\nu}, \bar{h}_{\nu}\right) \|_{\mathcal{R}_{\nu+1}^{\prime}} \dot{p}_{\nu} \frown\left(\lambda^{\prime}, 0,\left\lceil\dot{p}_{\nu}\right\rceil\right) \Vdash_{\mathcal{R}_{\nu, \lambda}^{\prime}} \sigma_{\xi} .
$$

Thus $\left(\nu, \bar{A}_{\nu}, \bar{h}_{\nu}\right) \frown \dot{p}_{\nu} \frown\left(\lambda^{\prime}, 0,\left\lceil\dot{p}_{\nu}\right\rceil\right) \| \sigma_{\xi}$ for each $\nu \in X$ and $\xi<\tau$.

There is a unique condition $\left(\lambda^{\prime}, \bar{A}, \bar{h}\right) \leq^{*}\left(\lambda^{\prime}, A, h\right)$ in $Q_{\lambda^{\prime}, \beta}$ so that

$$
X^{\prime}=\left\{\nu \in X: o(\nu)=\beta \text { and } \bar{A}_{\nu}=\bar{A} \cap \nu \text { and } \bar{h}_{\nu}=\bar{h} \uparrow \bar{A}_{\nu}\right\} \in U_{\lambda^{\prime}, \beta} .
$$

Take $X^{\prime \prime} \subset X^{\prime}$ in $U_{\lambda^{\prime}, \beta}$ so that the members $\nu$ of $X^{\prime \prime}$ agree on which way each of the sentences $\sigma_{\xi}$ is decided by $\left(\nu, \bar{A}_{\nu}, \bar{h}_{\nu}\right) \frown \dot{p}_{\nu} \frown\left(\lambda^{\prime}, 0,\left\lceil\dot{p}_{\nu}\right\rceil\right)$, and set $A^{\prime}=\bar{A} \cup X^{\prime \prime}$. Finally, set $h^{\prime}(\nu)=\bar{h}(\nu)$ if $o(\nu)<\beta$ and $\dot{p}_{\nu}$ if $o(\nu)=\beta$. Then the condition $\left(\lambda^{\prime}, \beta+1, A^{\prime}, h^{\prime}\right) \leq^{*}\left(\lambda^{\prime}, \beta, A, h\right)$ decides $\sigma_{\xi}$ for each $\xi<\tau$.

Subcase 3.3.1d. $\left(\lambda^{\prime}, q\right)=\left(\lambda^{\prime}, B, q^{\prime}\right)$, an instance of $U_{\lambda^{\prime}, 0} \times Q_{\lambda^{\prime}}^{*}$.

In this case $\left(\lambda^{\prime}, q^{\prime}\right)$ is an instance of $Q_{\lambda^{\prime}}^{*}$. Let $i: V \rightarrow M=\operatorname{ult}\left(V, U_{\lambda^{\prime}, 0}\right)$ be the canonical embedding, and notice that if $p \in P_{\lambda^{\prime}}$ then $\lambda^{\prime} \in i(B)$ and hence

$$
p^{\frown}\left(\lambda^{\prime}, 0, \eta\right) \leq\left(\lambda^{\prime}, q^{\prime}\right) \text { in } \mathcal{R}_{\lambda}^{\prime} * \dot{Q}_{\lambda^{\prime}}^{*} \Longleftrightarrow p \frown\left(\lambda^{\prime}, \eta\right) \leq i\left(\lambda^{\prime}, B, q^{\prime}\right) \text { in } \mathcal{R}_{\tau, i\left(\lambda^{\prime}\right)+1}^{M}
$$

for every $\eta \in \lambda^{\prime} \backslash \bar{\lambda}$. Now consider, for each $\xi<\tau$, the following sentence $\bar{\sigma}_{\xi}$, where $\dot{H}$ is the name for a $V$-generic subset of $\mathcal{R}_{\lambda+1}^{\prime}$ with $\left(\lambda^{\prime}, 0, \eta\right) \in \dot{H}$ :

$$
\exists r \frown\left(\lambda^{\prime}, 0, \eta\right) \in \dot{H} \quad M \models r\left\lceil\lambda^{\prime} \frown\left(\lambda^{\prime}, \eta\right) \Vdash i\left(\sigma_{\xi}\right) .\right.
$$

By the previous subcase 3.3.1c there is a condition $\left(\lambda^{\prime}, q^{\prime \prime}\right)=\left(\lambda^{\prime}, \beta^{\prime}, A^{\prime}, h^{\prime}\right) \leq^{*}$ $\left(\lambda^{\prime}, q^{\prime}\right)$ which decides the sentence $\bar{\sigma}_{\xi}$ for each $\xi<\tau$.

We now define, for each $\xi<\tau$, a set $B_{\xi} \subset B$ so that $\left(\lambda^{\prime}, B_{\xi}, q^{\prime \prime}\right) \| \sigma_{\xi}$. We can assume without loss of generality that $\left(\lambda^{\prime}, q^{\prime \prime}\right) \Vdash \bar{\sigma}_{\xi}$. It follows that if $p^{\frown}\left(\lambda^{\prime}, 0, \eta\right) \leq$ $\left(\lambda^{\prime}, q^{\prime \prime}\right)$, then $M \models p \frown\left(\lambda^{\prime}, \eta\right) \Vdash i\left(\sigma_{\xi}\right)$. However the relation " $p \frown\left(\lambda^{\prime}, 0, \eta\right) \leq\left(\lambda^{\prime}, q^{\prime \prime}\right)$ " is definable without using the ultrafilter $U_{\lambda^{\prime}, 0}$, and hence $\left\{(p, \eta): p \frown\left(\lambda^{\prime}, 0, \eta\right) \leq Q_{\lambda^{\prime}}^{*}\right.$ $\left.\left(\lambda, q^{\prime \prime}\right)\right\} \in M$. Thus $M$ satisfies the following statement:

$$
\forall p \frown\left(\lambda^{\prime}, 0, \eta\right) \leq_{Q_{\lambda^{\prime}}}\left(\lambda^{\prime}, q^{\prime \prime}\right) \quad p^{\frown}\left(\lambda^{\prime}, \eta\right) \Vdash i\left(\sigma_{\xi}\right) .
$$

Set $q^{\prime \prime} \downarrow \alpha=\left(\alpha, \beta^{\prime}, A^{\prime} \cap \alpha, h^{\prime}\lceil\alpha)\right.$ and let $B_{\xi}$ be the set of cardinals $\alpha \in B$ such that (in $V)$ :

$$
\forall p \frown(\alpha, 0, \eta) \leq_{Q_{\alpha}^{*}}\left(\alpha, q^{\prime \prime} \downarrow \alpha\right) \quad p^{\frown}(\alpha, \eta) \Vdash \sigma_{\xi} .
$$

Then $B_{\xi} \in U_{\lambda^{\prime}, 0}$ since $\lambda^{\prime} \in i\left(B_{\xi}\right)$, and $\left(\lambda^{\prime}, B_{\xi}, q^{\prime \prime}\right) \Vdash \sigma_{\xi}$.

Now set $B^{\prime}=\bigcap_{\xi<\tau} B_{\xi}$. Then $\left(\lambda^{\prime}, B^{\prime}, q^{\prime \prime}\right) \leq^{*}\left(\lambda^{\prime}, B, q^{\prime}\right)=\left(\lambda^{\prime}, q\right)$ and $\left(\lambda^{\prime}, B^{\prime}, q^{\prime \prime}\right) \|$ $\sigma_{\xi}$ for each $\xi<\tau$, as required.

This completes the proof of the final subcase of case 3.3.1, and we now turn to the limit case:

Case 3.3.2. $\lambda<\kappa$ is a limit cardinal. 
First note that if $p \in \mathcal{R}_{\tau, \lambda}^{\prime}$ and $\gamma=\lceil p\rceil$ is determined then $p \in \mathcal{R}_{\tau, \gamma+1}^{\prime}$, so $p$ has the $\tau$-Prikry property by the induction hypothesis.

We now prove the lemma for arbitrary $p \in \mathcal{R}_{\tau, \lambda}^{\prime}$ by induction on the length $n<\omega$ of $p$. If $n \leq 1$ then $\lceil p\rceil$ is determined, so we can assume $p=p_{0} \frown(\dot{\gamma}, \dot{q})$ where $p_{0}$ has length $n-1$.

Work for the moment in $V\left[C_{p_{0}}\right]$. Set $\eta=\sup C_{p_{0}}$, and suppose $(\dot{\gamma}, \dot{q})$ denotes $(\gamma, q)$ in $V\left[C_{p_{0}}\right]$. Then $(\gamma, q) \in \mathcal{R}_{\eta, \gamma+1}^{\prime V\left[C_{p_{0}}\right]}$, so by the induction hypothesis there is $p_{1}^{\prime} \leq^{*}(\gamma, q)$ so that $p_{1}^{\prime} \| \sigma_{\xi}$ in $\mathcal{R}_{\eta, \gamma+1}^{\prime V\left[C_{p_{0}}\right]}$ for every $\xi<\tau$.

Now return to working in $V$. Let $\dot{p}_{1}^{\prime}$ be a $C_{p_{0}}$-name for the condition $p_{1}^{\prime}$ above. Then $p_{0} \Vdash_{\mathcal{R}_{\lambda}^{\prime}} \forall \xi<\tau \dot{p}_{1}^{\prime} \|_{\mathcal{R}_{\dot{\eta}, \lambda}^{\prime}} \sigma_{\xi}$. By the induction hypothesis there is $p_{0}^{\prime} \leq^{*} p_{0}$ so that $p_{0}^{\prime} \|_{\mathcal{R}_{\lambda}^{\prime}} \dot{p}_{1}^{\prime} \Vdash_{\mathcal{R}_{\dot{\eta}, \lambda}^{\prime}} \sigma_{\xi}$ for all $\xi<\tau$, and $p_{0}^{\prime}$ decides the length and recipes of $\dot{p}_{1}^{\prime}$. Then $p_{0}^{\prime} \frown \dot{p}_{1}^{\prime} \leq^{*} p$, and $p_{0}^{\prime} \frown \dot{p}_{1}^{\prime} \|_{\mathcal{R}_{\lambda}^{\prime}} \sigma_{\xi}$ for all $\xi<\tau$, as required.

Case 3.3.3. $\lambda=\kappa$.

In this case the conditions in $\mathcal{R}_{\tau, \lambda}^{\prime}=\mathcal{R}_{\tau, \kappa}^{\prime}$ have length $\omega$. Pick $n_{0}<\omega$ large enough that domain $(p) \backslash \lambda_{n_{0}}=\left\{\lambda_{m}: m \geq \lambda_{n_{0}}\right\}$ and $\operatorname{cf}\left(o\left(\lambda_{m}\right)\right)>\tau$ for all $m>n_{0}$. We will show that $p \mid \lambda_{n_{0}}$ has the $\tau$-Prikry property in $\mathcal{R}_{\lambda_{n_{0}, \kappa}}^{\prime}$; as usual, this suffices because of the factorization $\mathcal{R}_{\tau, \lambda}^{\prime}=\mathcal{R}_{\tau, \lambda_{n_{0}}+1}^{\prime} * \dot{\mathcal{R}}_{\lambda_{n_{0}}, \kappa}^{\prime}$ together with the induction hypothesis for $\mathcal{R}_{\tau, \lambda_{n_{0}}+1}^{\prime}$. Also, since $p\left\lfloor\lambda_{n_{0}}\right.$ is $\tau^{+}$-closed it is sufficient to show that $p \mid \lambda_{n_{0}}$ has the Prikry property, that is, that for any single sentence $\sigma$ there is $p^{\prime} \leq p\left\lfloor\lambda_{n_{0}}\right.$ so that $p^{\prime} \| \sigma$.

First we define $\mathrm{a} \leq^{*}$-descending sequence of conditions $\left(p_{n}: n_{0} \leq n<\omega\right)$, with $p_{n_{0}}=p \mid \lambda_{n_{0}}$. Suppose that $n>n_{0}$ and $p_{n-1}$ has been defined. Let $\dot{p}_{n, 1}$ be a $\mathcal{R}_{\lambda_{n_{0}}, \lambda_{n}+1}^{\prime}$-term for a condition $p_{n, 1} \leq^{*} p_{n-1} \mid \lambda_{n}$ in $\mathcal{R}_{\lambda_{n}, \kappa}$ such that for any condition $\bar{p} \leq p_{n}\left\lceil\lambda_{n}+1\right.$, either $\bar{p}^{\frown} \dot{p}_{n}^{\prime} \| \sigma$ or else there is no $p^{\prime} \leq^{*} \dot{p}_{n}^{\prime}$ such that $\bar{p}^{\frown} p^{\prime} \| \sigma$.

Now pick $p_{n, 0} \leq^{*} p_{n}\left\lceil\lambda_{n}+1\right.$ so that $p_{n, 0} \| \exists \bar{p} \in \mathfrak{G}\left(C \cap \lambda_{n}+1\right) \bar{p}^{\frown} \dot{p}_{n, 1} \Vdash \sigma$, and set $p_{n+1}=p_{n, 0} \frown \dot{p}_{n, 1}$.

We claim that the condition $p^{\prime}=\bigwedge_{n_{0}<n<\omega} p_{n}$ decides $\sigma$. To see this, pick any condition $\bar{p} \leq p^{\prime}$ such that $\bar{p} \| \sigma$. Then there is some $n<\omega$ so that $\bar{p} \mid \lambda_{n} \leq{ }^{*}$ $p^{\prime}\left|\lambda_{n} \leq^{*} p_{n}\right| \lambda_{n}$. It follows that $\left(\bar{p} \mid \lambda_{n}+1\right) \frown p_{n} \mid \lambda_{n} \| \sigma$, and since $\bar{p} \mid \lambda_{n}+1 \leq$ $p_{n}\left\lceil\left(\lambda_{n}+1\right)\right.$ it follows that $p_{n} \| \sigma$. Since $p^{\prime} \leq^{*} p_{n}$, it follows that $p^{\prime} \| \sigma$.

This completes the case $\lambda=\kappa$, and hence the proof of lemma 3.3.

Remark 3.4. We will use this same proof in section 4 for the proof of the $\tau$-Prikry property for the forcing $\mathbf{R}_{\tau, \lambda}$ of the main theorem 1.1 The necessary modifications will be described in detail there, but we summarize them here: In order of increasing difficulty, we will need to (1) omit the final case $\lambda=\kappa$, since all conditions are finite, (2) verify that the side condition $\vec{B}$ does not interfere with the constructions used in this proof, and (3) use the sequence $\vec{B}$ to prove the analog of lemma 3.1 for $\mathbf{R}_{\kappa+1}$.

Lemma 3.5. Suppose $C$ is $\mathcal{R}_{\kappa}^{\prime}$-generic and $x \in \mathcal{P}(\lambda) \cap V[C]$ for some $\lambda<\kappa$. Then $x \in V[C \cap \lambda]$.

Proof. Let $\dot{x}$ be a name for $x$ such that $\Vdash \dot{x} \subset \lambda$, and let $p \in \mathfrak{G}(C)$ force that $\sup (C \cap \lambda)=\gamma \leq \lambda$. Then $p$ can be written $p=p_{0} \frown \dot{p}_{1}$ where $\left\lceil p_{0}\right\rceil=\gamma \leq \lambda \leq\left\lfloor p_{1}\right\rfloor$. Now work in $V\left[C_{p_{0}}\right]$. By lemma $3.3 p_{1}$ has the $\lambda^{+}$-Prikry property and hence there 
is $p_{1}^{\prime} \leq^{*} p_{1}$ which decides the sentence $\xi \in \dot{x}$ for each $\xi<\lambda$. Then $p_{1}^{\prime} \Vdash_{\mathcal{R}^{\prime} / p_{0}} \dot{x}=$ $\left\{\xi<\lambda: \dot{p}_{1}^{\prime} \Vdash_{\mathcal{R}^{\prime} / \dot{p}_{1}} \xi \in \dot{x}\right\} \in V\left[C_{p_{0}}\right]=V[C \cap \lambda]$.

Corollary 3.6. No cardinals are collapsed by $\mathcal{R}_{\kappa}$.

Proof. No cardinal greater than $\kappa^{+}$is collapsed since $\left|\mathcal{R}_{\kappa}^{\prime}\right|=\kappa^{+}$. No cardinal $\eta \leq \kappa$ is collapsed by lemma 3.5 .

To see that $\kappa^{+}$is not collapsed, let $\dot{f}$ be an arbitrary $\mathcal{R}_{\kappa}^{\prime}$ name for a function from $\kappa$ into $\kappa^{+}$. Define $\mathrm{a} \leq{ }^{*}$-decreasing sequence of conditions $p_{n}$ so that if $p^{\prime} \leq p_{n}$ is any condition such that $p^{\prime}\left|\lambda_{n} \leq^{*} p_{n}\right| \lambda_{n}$ and $p^{\prime} \Vdash \dot{f}(\nu)=\eta$ for some $\nu<\lambda_{n}$, then $p^{\prime}\left|\lambda_{n}+1 \frown p_{n}\right| \lambda_{n} \Vdash \dot{f}(\nu)=\eta$. Then $\bigwedge_{n<\omega} p_{n}$ forces that range $\dot{f} \subset\{\eta: \exists n<$ $\left.\omega \exists \nu<\lambda_{n} \exists p^{\prime} \in \mathcal{R}_{\lambda_{n}+1}^{\prime} p^{\prime} p_{n} \mid \lambda_{n} \Vdash \dot{f}(\nu)=\eta\right\}$, but this set has cardinality $\kappa$ and hence is bounded in $\kappa^{+}$.

Corollary 3.7. Suppose that $C$ is $\mathcal{R}_{\kappa}^{\prime}$-generic, and let $\kappa_{n}$ be the unique ordinal in the interval $\lambda_{n-1}<\kappa_{n}<\lambda_{n}$ such that there is a condition $p \in \mathfrak{G}(C)$ having an instance $\left(\kappa_{n}, \bar{\kappa}_{n}\right)$ (spawned by $U_{\lambda_{n}, 0} \times Q_{\lambda_{n}}^{*}$ ) of $P_{\kappa_{n}}$. Then for each $n<\omega, \kappa_{n}$ is regular in $V[C]$, and $C \cap \kappa_{n}$ is a closed and unbounded subset of $\kappa_{n} \cap E$.

Proof. The assertion that $C \cap \kappa_{n}$ is a closed and unbounded subset of $\kappa_{n} \cap E$ is immediate from the definition of the forcing. To see that $\kappa_{n}$ is regular, let $\tau$ be any function in $V[C]$ which maps some ordinal $\eta<\kappa_{n}$ into $\kappa_{n}$. Then $\tau \in V\left[C \cap \kappa_{n}\right]$ by lemma 3.5, and it follows from lemma 3.1] that there is $p \in \mathcal{R}_{\kappa_{n}+1}^{\prime} \cap \mathfrak{G}\left(C \cap \kappa_{n}+1\right)$ such that $\tau \in V\left[C_{p\left\lceil\kappa_{n}\right.}\right]=V[C \cap \gamma]$ where $\gamma=\left\lceil p\left\lceil\kappa_{n}\right\rceil<\kappa_{n}\right.$. But then $p$ forces that $\tau$ is bounded in $\kappa_{n}$, since $\kappa_{n}$ is inaccessible and $\left|\mathcal{R}^{\prime} /\left(p \mid \kappa_{n}\right)\right| \leq \gamma^{+}<\kappa_{n}$.

This finishes the proof of theorem 1.5.

\section{The Proof of the MaIN theorem 1.1}

Now we turn to the proof of the direction $(1) \Longrightarrow(2)$ of theorem 1.1 using the forcing $\mathbf{R}_{\kappa+1}$ defined in definition 2.5. This involves proving the analog for this forcing of results from section 3 , notably of lemmas 3.1 and 3.3 and then of proving that $\kappa$ is still measurable in the generic extension $V[C]$.

It was observed previously that three things are necessary to adapt the proof of lemma 3.3 to this forcing. The first, omitting the case $\lambda=\kappa$, does not require further comment. The third, proving the analog of the distributivity lemma 3.1 will be addressed by lemmas 4.4 and 4.6 in subsection 4.1 below. In order to facilitate the second, showing that the presence of the measure one sequence $\vec{B}$ does not interfere with the constructions used in the proof of lemma 3.3, we begin with some observations about the role of this sequence.

We have already established the convention of writing $\vec{B}^{\prime} \subset \vec{B}$ when $B_{\gamma}^{\prime} \subset B_{\gamma}$ for each $\gamma$ in their common domain. Similarly, if $\vec{B}^{\xi}$ is a measure one sequence on the same interval for each $\xi<\eta$, then we will write $\vec{B}^{\prime}=\bigcap_{\xi<\eta} \overrightarrow{B^{\xi}}$ and $\vec{B}^{\prime \prime}=\triangle_{\xi} B^{\xi}$ for the sequences with members $B_{\gamma}^{\prime}=\bigcap_{\xi} B_{\gamma}^{\xi}$ and $B_{\gamma}^{\prime \prime}=\left\{\nu<\gamma: \nu \in \bigcap_{\xi<\nu} B_{\nu}^{\xi}\right\}$, respectively.

Proposition 4.1. 1. If $\tau \leq \eta<\lambda$ and $\vec{B}^{\xi}$ is a measure one sequence on the interval $(\eta, \lambda)$ for each $\xi<\tau$, then $\bigcap_{\xi} \vec{B}^{\xi}$ is also a measure one sequence on the interval $(\eta, \lambda)$. 
2. If $0 \leq \eta<\lambda$ and $\vec{B}^{\xi}$ is a measure one sequence on an interval $(\eta, \lambda)$ for each $\xi<\lambda$, then $\triangle_{\xi<\lambda} \vec{B}^{\xi}$ is also a measure one sequence on $(\eta, \lambda)$.

The first clause implies that if a condition $p \in \mathcal{R}_{\lambda}$ is $\tau$-closed then so is the condition $(p, \vec{B}) \in \mathbf{R}_{\lambda}$, regardless of $\vec{B}$. The second clause implies that proposition 2.13 asserting that $Q_{\lambda}$ is diagonally $\lambda$-closed, is still valid in $\mathbf{R}_{\lambda+1}$.

Proposition 4.2. If $\lambda$ is a cardinal then $\mathbf{R}_{\lambda}$ has the $\lambda^{+}$-chain condition.

Proof. Any two conditions $(p, \vec{B})$ and $\left(p^{\prime}, \vec{B}^{\prime}\right)$ with $p=p^{\prime}$ are compatible, and $\left|\mathcal{R}_{\lambda}\right| \leq \lambda$.

Proposition 4.3. Suppose that $\left(p^{\prime}, \vec{B}^{\prime}\right) \leq^{*}(p, \vec{B})$. Then for any $\vec{D} \subset \vec{B}$ there is $\left(p^{\prime \prime}, \vec{B}^{\prime \prime}\right) \leq^{*}\left(p^{\prime}, \vec{B}\right)$ such that $\left(p^{\prime \prime}, \vec{B}^{\prime \prime}\right) \leq^{*}(p, \vec{D})$.

Furthermore, if $\left(p^{\prime}, \vec{B}^{\prime}\right) \leq(p, \vec{B})$ and there is no instance of $P_{\gamma}$ in $p^{\prime}$ with $\gamma \in$ domain $\left(p^{\prime}\right) \backslash$ domain $(p)$, then there is $\left(p^{\prime \prime}, \vec{B}^{\prime \prime}\right) \leq{ }^{*}\left(p^{\prime}, \vec{B}\right)$ such that $\left(p^{\prime \prime}, \vec{B}^{\prime \prime}\right) \leq$ $(p, \vec{D})$.

Proof. We can take $\vec{B}^{\prime \prime}=\vec{D} \cap \vec{B}^{\prime}$ and domain $\left(p^{\prime \prime}\right)=\operatorname{domain}\left(p^{\prime}\right)$. Now for every $\gamma \in \operatorname{domain}\left(p^{\prime}\right) \backslash$ domain $(p)$ such that $p^{\prime}$ has an instance $(\gamma, B, q)$ of $U_{\gamma, 0} \times Q_{\gamma}^{*}$, replace this instance in $p^{\prime \prime}$ with $\left(\gamma, B_{\gamma}^{\prime \prime} \cap B, q\right)$.

We conclude these remarks with a final bit of notation. Notice that the only effect a member $B_{\gamma}$ of the sequence $\vec{B}$ in a condition $\mathbf{p}=(p, \vec{B})$ can have is on an instance $\left(\gamma,(B, q), \vec{B}^{\prime}\right)$ of $U_{\gamma, 0} \times Q_{\gamma}^{*}$ in some condition $\mathbf{p}^{\prime} \leq \mathbf{p}$. Since any such instance must occur in the interval $\lfloor(\lambda, q)\rfloor<\gamma<\lceil(\lambda, q)\rceil=\lambda$ associated with some coordinate $p_{\lambda}=(\lambda, q)$ of $p$, it is useful to divide $\vec{B}$ into subsequences associated with the coordinates of $p$. In order to make this explicit we will modify our notation for members of $\mathbf{R}_{\lambda}$; for example, instead of writing $\mathbf{p}=\left(p_{0} \frown\left(\gamma_{1}, q_{1}\right) \frown\left(\gamma_{2}, q_{2}\right), \vec{B}\right)$ we will write

$$
\mathbf{p}=\mathbf{p}_{0} \frown\left(\gamma_{1}, q_{1}, \vec{B}^{1}\right) \frown\left(\gamma_{2}, q_{2}, \vec{B}^{2}\right) .
$$

Here $\mathbf{p}_{0}=\left(p_{0}, \vec{B}^{0}\right)$, as a member of $\mathbf{R}_{\left\lceil p_{0}\right\rceil+1}$, contains its own measure one sequence, and $\vec{B}^{i}$ for $i=1,2$ are measure one sequences on the intervals $\left(\eta_{i}, \gamma_{i}\right)$, where $\eta_{i}=\left\lfloor\left(\gamma_{i}, q_{i}\right)\right\rfloor$. As a further simplification we will frequently write $(\lambda, q, \vec{B})$, rather than writing $(\lambda, q, \vec{B}\lceil(\lfloor q\rfloor, \lambda))$ to explicitly show the restriction of $\vec{B}$ to the correct interval.

4.1. The Prikry property. The next two lemmas are, together, the analog of lemma 3.1 The second is the direct analog of lemma 3.1, while the first is needed to deal with the case of $\tau=1$. This is necessary because, in contrast to the forcing $\mathcal{R}_{\lambda+1}^{\prime}$, where $p \leq(\lambda, \eta)$ implies $p \leq^{*}(\lambda, \eta)$, the forcing $\mathcal{R}_{\lambda+1}$ can have non-direct extensions of the form

$$
(\gamma, \bar{\gamma}) \frown\left(\lambda, \lambda^{\prime}\right) \leq\left(\lambda^{\prime},(B, q)\right) \frown\left(\lambda, \lambda^{\prime}\right) \leq^{*}(\lambda, \bar{\gamma})
$$

factoring through an instance $(B, q)$ of $U_{\lambda^{\prime}, 0} \times Q_{\lambda^{\prime}}^{*}$ such that $\gamma \in B$. The sequence $\vec{B}$ was introduced in order to deal with this problem.

Lemma 4.4. Suppose that $\lambda$ is inaccessible and $\bar{\lambda}<\lambda$. Then for any dense open subset $\mathcal{D}$ of $\mathbf{P}_{\lambda}$ there is a condition $\mathbf{p}^{-}\left(\lambda, \eta, \vec{B}^{\prime}\right) \leq^{*}(\lambda, \bar{\lambda}, \vec{B})$ such that $\left\{\mathbf{p}^{\prime}\right.$ : 
$\left.\mathbf{p}^{\prime}-\left(\lambda, \eta, \overrightarrow{B^{\prime}}\right) \in \mathcal{D}\right\}$ is dense in $\mathbf{R} / \mathbf{p}$. Thus

$$
\mathbf{p}^{\frown}\left(\lambda, \eta^{\prime}, \vec{B}^{\prime}\right) \Vdash \exists \mathbf{p}^{\prime} \in \mathfrak{G}\left(C_{p}\right) \mathbf{p}^{\prime} \frown\left(\lambda, \eta^{\prime}, \vec{B}^{\prime}\right) \in \mathcal{D} .
$$

Proof. First, note that it is sufficient to prove that

$$
\mathcal{D}^{\prime}=\left\{\mathbf{p}^{\prime}: \exists \eta_{\mathbf{p}^{\prime}}, \vec{B}_{\mathbf{p}^{\prime}} \mathbf{p}^{\prime}-\left(\lambda, \eta_{\mathbf{p}^{\prime}}, \vec{B}_{\mathbf{p}^{\prime}}\right) \in \mathcal{D}\right\}
$$

is dense in $\mathbf{R} / \mathbf{p}$, since $\eta=\sup _{\mathbf{p}^{\prime}} \eta_{\mathbf{p}^{\prime}}<\lambda$ and $\vec{B}^{\prime}=\bigcap_{\mathbf{p}^{\prime}} \vec{B}_{\mathbf{p}^{\prime}}$ is a measure one sequence on the interval $(\eta, \lambda)$.

Note that an extension $\mathbf{p}-\left(\lambda, \xi, \vec{B}^{\prime}\right) \leq(\lambda, \vec{\lambda}, \vec{B})$ is always direct unless $p$ contains an instance of $P_{\gamma}$ for some ordinal $\gamma$. Let $\chi(\mathbf{p})$ be the largest such ordinal $\gamma$, or set $\chi(\mathbf{p})=0$ if the extension is direct. Note that $\chi(\mathbf{p})$ will actually be a term; however for any condition $\mathbf{p}$ there is a condition $\mathbf{p}^{\prime} \leq \mathbf{p}\lceil\chi(\mathbf{p})$ which determines the value of the term $\chi(\gamma)$. Thus we can assume without loss of generality that the value of $\chi(\gamma)$ is determined.

Let $\mathcal{D}^{*}$ be the set of $\mathbf{p} \in \mathbf{R}_{\lambda}$ such that $\mathcal{D}^{\prime}$ is dense in $\mathbf{R} / \mathbf{p}$, and note that $\mathcal{D}^{*} \neq \varnothing$ since $\mathcal{D}^{*} \supset \mathcal{D}^{\prime} \supset\{\mathbf{p} \mid \lambda: \mathbf{p} \in \mathcal{D}\}$.

Claim. There is a measure one sequence $\vec{B}^{\prime} \subset \vec{B}$ with the following property: Suppose $\mathbf{p} \in \mathcal{D}^{*}$ and $\mathbf{p}-(\lambda, \xi) \leq\left(\lambda, \bar{\lambda}, \vec{B}^{\prime}\right)$. If $\chi(\mathbf{p}) \neq 0$ then there is $\mathbf{p}^{\prime} \frown\left(\lambda,\left\lceil\mathbf{p}^{\prime}\right\rceil\right) \leq$ $\left(\lambda, \bar{\lambda}, \vec{B}^{\prime}\right)$ such that $\mathbf{p}^{\prime} \in \mathcal{D}^{*}$ and $\chi\left(\mathbf{p}^{\prime}\right)<\chi(\mathbf{p})$.

Proof. First we define $B_{\gamma}^{\prime}$ for an arbitrary $\gamma \in$ domain $\vec{B}$. For each $\eta \in B_{\gamma}$ define $X_{\eta}$ to be the set of conditions $\mathbf{p} \in \mathbf{R}_{\bar{\lambda}, \eta}$ such that there is a measure one sequence $\vec{B}^{\mathbf{p}}$ and a condition $\mathbf{p}^{*} \in \mathbf{R}_{\gamma, \lambda}$ satisfying the following two conditions:

$$
\begin{aligned}
\mathbf{p}^{\frown} & \frown\left(\eta,\lceil\mathbf{p}\rceil, \vec{B}^{\mathbf{p}}\right) \frown \mathbf{p}^{*} \in \mathcal{D}^{*}, \\
\mathbf{p}^{\frown}\left(\eta,\lceil\mathbf{p}\rceil, \vec{B}^{\mathbf{p}}\right) \frown \mathbf{p}^{*} & \leq{ }^{*} \mathbf{p} \frown\left(\eta,\lceil\mathbf{p}\rceil, \vec{B}^{*}\right) \frown(\lambda, \gamma) \\
& \leq \mathbf{p}^{\frown}\left(\gamma,\left(B_{\gamma}, 0,\lceil\mathbf{p}\rceil\right), \vec{B}\right) \frown(\lambda, \gamma) \leq^{*} \mathbf{p}^{\frown}(\lambda, \bar{\lambda}, \vec{B}) .
\end{aligned}
$$

If we set $\vec{B}^{\eta}=\triangle_{\mathbf{p}} \vec{B}^{\mathbf{p}}$ then we can assume without loss of generality that $\vec{B}^{\mathbf{p}}=\vec{B}^{\eta}$ for all $\mathbf{p} \in X_{\eta}$. Notice in addition that we would not have gained any generality by using an arbitrary ordinal $\xi$ in place of $\lceil\mathbf{p}\rceil$ in (10b), since for any condition $\mathbf{p}^{\frown}\left(\eta, \xi, \overrightarrow{B^{\mathbf{p}}}\right)$, we can find $\mathbf{p}^{\prime}$ so that $\mathbf{p}^{\prime} \frown\left(\eta,\lceil\mathbf{p}\rceil, \overrightarrow{B^{\mathbf{p}}}\right) \leq^{*} \mathbf{p} \frown\left(\eta, \xi, \overrightarrow{B^{\mathbf{p}}}\right)$ by simply adding an ordinal $\xi^{\prime}>\xi$ to the domain of $\mathbf{p}$.

There is a unique set $X^{\gamma} \subset \mathbf{R}_{\gamma}$ and sequence $\vec{B}_{\gamma}^{*}$ such that

$$
B^{\prime}=\left\{\eta \in B_{\gamma}: X_{\eta}=X^{\gamma} \cap V_{\eta} \text { and } \vec{B}^{\eta}=\vec{B}_{\gamma}^{*}\lceil\eta\} \in U_{\gamma, 0} .\right.
$$

We set $B_{\gamma}^{\prime}=B^{\prime}$.

This completes the definition of the sequence $\vec{B}^{\prime}$, and we now show that $\vec{B}^{\prime}$ satisfies the claim. Suppose that $\mathbf{p}^{-}(\lambda, \xi) \leq\left(\lambda, \bar{\lambda}, \vec{B}^{\prime}\right)$ is a condition such that $\mathbf{p} \in \mathcal{D}^{*}$ and $0<\eta=\chi(\mathbf{p})$. Thus there is $\gamma>\eta$ so that

$$
\begin{aligned}
& \mathbf{p}^{\frown}\left(\lambda,\lceil\mathbf{p}\rceil, \vec{B}^{\prime}\right)=\mathbf{p}_{0} \frown\left(\eta, \bar{\eta}, \vec{B}_{\gamma}^{*}\right) \frown \mathbf{p}_{1} \frown\left(\lambda,\lceil\mathbf{p}\rceil, \vec{B}^{\prime}\right) \\
& \leq^{*} \mathbf{p}_{0} \frown\left(\eta, \bar{\eta}, \vec{B}_{\gamma}^{*}\right) \frown\left(\lambda, \gamma, \vec{B}^{\prime}\right) \\
& \leq \mathbf{p}_{0} \frown\left(\gamma,\left(B_{\gamma}^{\prime}, 0, \bar{\eta}\right), \vec{B}_{\gamma}^{*}\right) \frown\left(\lambda, \gamma, \vec{B}^{\prime}\right) \\
& \leq^{*} \mathbf{p}_{0} \frown\left(\lambda, \bar{\lambda}, \vec{B}^{\prime}\right) \text {. }
\end{aligned}
$$


Then $\eta \in B_{\gamma}^{\prime}$ by (11), and it follows that $\mathbf{p}_{0} \in X_{\gamma}$. Thus, for each $\eta>\left\lceil\mathbf{p}_{0}\right\rceil$ in $B_{\gamma}^{\prime}$ there is a condition $\mathbf{p}_{\eta}^{*}$ so that $\mathbf{p}_{0} \frown\left(\eta,\left\lceil\mathbf{p}_{0}\right\rceil, \vec{B}_{\gamma}^{*}\lceil\eta) \frown \mathbf{p}_{\eta}^{*} \in \mathcal{D}^{*}\right.$. Now shrink to a set $B^{\prime \prime} \subset B_{\gamma}^{\prime}$ in $U_{\gamma, 0}$ such that the length of $p_{\eta}^{*}$ and the recipes used by $p_{\eta}^{*}$ are the same for all $\eta \in B^{\prime \prime}$, and let $\dot{\mathbf{p}}^{*}$ be a $C \cap \gamma$-term so that

$$
\mathbf{p}_{0} \subsetneq\left(\eta,\left\lceil\mathbf{p}_{0}\right\rceil, \vec{B}^{\prime \prime}\right) \Vdash \dot{\mathbf{p}}^{*}=\mathbf{p}_{\eta}^{*}
$$

for all $\eta \in B^{\prime \prime}$. Finally, let $\mathbf{p}^{\prime}=\mathbf{p}_{0} \frown\left(\gamma,\left(B^{\prime \prime}, 0,\left\lceil\mathbf{p}_{0}\right\rceil\right), \vec{B}^{\prime \prime}\right) \frown \dot{\mathbf{p}}^{*}$. Then $\chi\left(\mathbf{p}^{\prime}\right) \leq$ $\left\lceil p_{0}\right\rceil<\eta=\chi(\mathbf{p})$, and $\mathbf{p}^{\prime} \in \mathcal{D}^{*}$ since for any condition $\mathbf{p}^{\prime \prime} \leq \mathbf{p}^{\prime}$ there is some $\eta \in B_{\gamma}^{\prime}$ such that the condition $\mathbf{p}_{0} \frown\left(\eta,\left\lceil\mathbf{p}_{0}\right\rceil, \vec{B}^{\gamma}\right) \in \mathcal{D}^{*}$ is comparable to $\mathbf{p}^{\prime \prime}$. This finishes the proof of the claim.

Now take $\mathbf{p}^{-}(\lambda, \bar{\lambda}) \leq\left(\lambda, \bar{\lambda}, \vec{B}^{\prime}\right)$ with $\mathbf{p} \in \mathcal{D}^{*}$ and $\chi(\mathbf{p})$ as small as possible. It follows from the claim that $\chi(\mathbf{p})=0$, that is, $\mathbf{p}^{-}(\lambda, \bar{\lambda}) \leq^{*}\left(\lambda, \bar{\lambda}, \vec{B}^{\prime}\right)$.

Remark 4.5. Notice that this proof of lemma 4.4 requires the use of backward Easton iteration in order to construct the term $\dot{\mathbf{p}}^{*}$ in (12) above. This is the only place in this paper where the use of backward Easton forcing is required (although avoiding it in the proof of lemma 3.1 requires a slight strengthening of the hypothesis). See [7] for details.

The next lemma is the direct analog of lemma 3.1 from section 3 ,

Lemma 4.6. Suppose that $\tau<\lambda \in D$ and $(\lambda, \bar{\lambda}, \vec{B}) \in \mathbf{R}_{\lambda+1}$. Then for any sequence $\overrightarrow{\mathcal{D}}=\left(\mathcal{D}_{\xi}: \xi<\tau\right)$ of dense open sets of conditions in $\mathbf{R}_{\lambda+1}$ there is a condition $\mathbf{p} \in \mathbf{R}_{\lambda}$ so that $\mathbf{p}^{-}(\lambda,\lceil p\rceil) \leq^{*}(\lambda, \bar{\lambda}, \vec{B})$ and $\mathcal{D}_{\xi}^{\prime}=\left\{\mathbf{p}: \exists \eta^{\prime}, \vec{B}^{\prime} \mathbf{p}^{\frown}\left(\lambda, \eta^{\prime}, \vec{B}^{\prime}\right)\right\}$ is dense in $\mathbf{R} / \mathbf{p}$ for for all $\xi<\tau$.

Hence there is $\left(\lambda, \eta^{\prime}, \vec{B}^{\prime}\right) \leq(\lambda, \bar{\lambda}, \vec{B})$ so that $\mathbf{p}^{-}\left(\lambda, \eta^{\prime}, \vec{B}^{\prime}\right) \Vdash \forall \xi<\tau \exists \mathbf{p}^{\prime} \in$ $\mathfrak{G}\left(C_{\mathbf{p}}\right) \mathbf{p}^{\prime} \frown\left(\lambda, \eta^{\prime}, \vec{B}^{\prime}\right) \in \mathcal{D}_{\xi}$.

Proof. The proof is a straightforward modification of the proof of lemma 3.1. The condition $\mathbf{p}$ will be $\left(\gamma,(A, h), \vec{B}^{1}\right) \frown\left(\lambda, \eta^{\prime}, \vec{B}^{2}\right)$, where $(\gamma,(A, h))$ and $\left(\lambda, \eta^{\prime}\right)$ are as in lemma 3.1. except that lemma 4.4 is needed to define $h(\nu)$. To define $\vec{B}^{1}$ and $\vec{B}^{2}$, let $X_{\gamma} \prec \mathrm{H}_{\lambda^{+}}$be as in the proof of lemma 3.1 and pick $\vec{B}^{\mathbf{p}} \in X_{\gamma}$ for each $\mathbf{p} \in X_{\gamma}$ so that $\mathbf{p} \frown(\lambda, \gamma, \vec{B} \mathbf{p}) \in \mathcal{D}_{\xi}$ for each $\xi<\tau$ and $\mathbf{p} \in \mathcal{D}_{\xi}^{\prime}$. Now set $\vec{B}^{1}=\triangle_{\mathbf{p} \in X_{\gamma}}\left(\vec{B}^{\mathbf{p}}\lceil\gamma)\right.$ and $\vec{B}^{2}=\bigcap_{\mathbf{p} \in X_{\gamma}}\left(\overrightarrow{B^{\mathbf{p}}}\lfloor\gamma)\right.$.

Lemma 4.7. If $\tau<\lambda \leq \kappa+1$, then $\mathbf{R}_{\tau, \lambda}$ has the $\tau^{+}$-Prikry property.

Proof. We have already covered two of the three needed modifications to the proof of lemma 3.3 by omitting the special case for $\lambda=\kappa$, and by proving lemma 4.6 to be used in place of lemma 3.1 for subcase $3.3 .1 \mathrm{a}$ for the recipe $P_{\lambda^{\prime}}$.

The final modification, incorporating the measure one sequence $\vec{B}$ into the argument, does not require any actual changes, but it does require verifying that the inclusion of the sequence $\vec{B}$ does not adversely affect the argument. As a first step in this verification, notice that by proposition 4.3 the sequence $\vec{B}$ does not affect the direct extension ordering $\left(p^{\prime}, \vec{B}^{\prime}\right) \leq^{*}(p, \vec{B})$ in an essential way, except through the requirement that $\vec{B}^{\prime} \subset \vec{B}$. By proposition 4.1 it follows that $\leq^{*}$ has the same closure properties as the ordering $p^{\prime} \leq^{*} p$ has in $\mathcal{R}_{\kappa}$. Thus all of the arguments remain valid except possibly those involving the forcing order $\leq$ as well as the direct extension $\leq^{*}$. Such arguments only occur in cases $3.3 .1 \mathrm{~b}$ and $3.3 .1 \mathrm{~d}$. By the 
second paragraph of proposition 4.3 there is still no problem unless the non-direct extension involves adding an instance of a recipe $P_{\gamma}$ for some $\gamma \notin \operatorname{domain}(p)$.

In case $3.3 .1 \mathrm{~b}$, the only non-direct extension we need to construct comes from a one-step extension using definition [2.12(3) for the recipe $Q$. Since $h(\nu)$ is required by that definition to be $\nu$-closed, it cannot contain any instances of $P_{\gamma}$; hence the measure one sequence will not be an obstacle.

The argument in case $3.3 .1 \mathrm{~d}$ does use direct extensions involving an instance of $P_{\gamma}$; however this instance is spawned from the instance of $U_{\lambda^{\prime}, 0} \times Q_{\lambda^{\prime}}^{*}$ already present in the given condition $p$, and hence also is not affected by the measure one sequence.

Thus the proof of lemma 3.3 carries over to prove the current lemma.

As in section 3, the Prikry property implies the following proposition:

Proposition 4.8. If $x \subset \eta<\lambda$ and $x \in V[C]$ where $C$ is $\mathbf{R}_{\lambda}$ generic, then $x \in V[C \cap \eta]$.

Proposition 4.9. If $\lambda$ is a limit cardinal, then $\mathbf{P}_{\lambda}$ has the $\lambda^{+}$-chain condition.

Proof. Let $A \subset \mathbf{R}_{\lambda}$ be an antichain. As with Prikry forcing, any two conditions $\mathbf{p}^{-}(\lambda, \eta, \vec{B})$ and $\mathbf{p}^{\prime} \frown\left(\lambda, \eta^{\prime}, \vec{B}^{\prime}\right)$ in $A$ must have $\mathbf{p} \neq \mathbf{p}^{\prime}$. Furthermore, by proposition [2.9] we may assume that for each condition $\mathbf{p}^{-}((\lambda, \eta), \vec{B}) \in A$ the cardinals in $\operatorname{domain}(p)$ are determined, so that $\mathbf{p} \in \mathbf{R}_{\lambda^{\prime}}$ for some $\lambda^{\prime}<\lambda$. Thus there are only $\lambda$ many choices for $\mathbf{p}$.

In particular, $\mathbf{R}_{\kappa+1}=\mathbf{P}_{\kappa}$ has the $\kappa^{+}$-chain condition.

Lemma 4.10. If $C \subset \lambda$ is $\mathbf{R}_{\lambda}$-generic, then the cardinals of $V[C]$ are the same as those of $V$.

Proof. Suppose the contrary, and let $\eta^{+}$be the least cardinal collapsed. By proposition 4.8 it follows that $\eta^{+}$is collapsed in $V[C \cap \eta]$. Since $\left|\mathbf{R}_{\lambda}\right|=\lambda$ we must have $\eta=\sup (C \cap \eta)$, so that $C \cap \eta$ is $\mathbf{R}_{\eta+1}$-generic, and $\eta$ is regular in $V$ by the covering lemma. Furthermore the recipe used at $\eta$ must be $Q_{\eta}$, since lemma 4.9 implies that it is not $P_{\eta}$ or (since it is equivalent to $P_{\eta}$ ) $Q_{\eta}^{*}$, and $U_{\eta, 0} \times Q_{\eta}^{*}$ would force $\sup (C \cap \eta)<\eta$. It follows that $\eta$ is singular in $V[C \cap \eta]$, but then the covering lemma implies that $\eta^{+}$is not collapsed.

The use of the covering lemma can be eliminated as in the proof of lemma 3.6

4.2. Measurability. It only remains to prove that $\kappa$ remains measurable in $V[C]$ :

Theorem 4.11. If $o(\kappa)=\kappa$ and $C \subset \kappa$ is $\mathbf{R}_{\kappa+1}$-generic, then $\kappa$ is measurable in $V[C]$.

Proof. Let $i: V \rightarrow M=\operatorname{ult}\left(V, U_{\kappa, 0}\right)$. Then $i\left(\mathbf{R}_{\kappa+1}\right)$ can be factored $i\left(\mathbf{R}_{\kappa+1}\right)=$ $\mathbf{R}_{\kappa+1} * \dot{\mathbf{R}}_{\kappa, i(\kappa)+1}^{M}$ and the set $C$ is $\mathbf{R}_{\kappa+1}$-generic over $M$. We will work in $V[C]$, and consider the forcing notion $\mathbf{R}_{1}$ over $M[C]$, which is equivalent to $\mathbf{R}_{\kappa, i(\kappa)+1}^{M[C]}$ but uses the recipe $Q_{i(\kappa)}^{*}$ instead of $P_{i(\kappa)}$ at $i(\kappa)$.

Let $\mathcal{A}$ be the set of antichains (not necessarily maximal) of $\mathbf{R}_{1}$ in $M[C]$. Then $|\mathcal{A}|^{M[C]}=i\left(\kappa^{+}\right)$since $\mathbf{R}_{1}$ has the $i\left(\kappa^{+}\right)$-chain condition in $M[C]$ by lemma 4.9 and hence $|\mathcal{A}|^{V}=\kappa^{+}$. Now the condition $\mathbf{1}^{*}=\left(i(\kappa),(0, \kappa), i\left(\vec{B}^{\mathbf{1}}\right)\right)$ has the Prikry property in $M[C]$, and it is $\kappa^{+}$-closed in $V[C]$ since it is $\kappa^{+}$-closed in $M[C]$, so we 
can define a $\leq^{*}$-descending sequence of conditions $q_{\xi} \leq^{*}(i(\kappa),(0, \kappa))$ for $\xi<\kappa^{+}$ so that for each $a \in \mathcal{A}$ there is some $\xi<\kappa^{+}$and $\vec{B}$ on the interval $(\kappa, i(\kappa))$ so that $M[C] \models\left(i(\kappa), q_{\xi}, \vec{B}\right) \|_{\mathbf{R} / \mathbf{1}^{*}} \mathfrak{G}(\dot{C}\lfloor\kappa) \cap a \neq \varnothing$.

Let $G^{*}$ be the set of conditions $\mathbf{p} \in \mathbf{R} / \mathbf{1}^{*}$ such that $\left(i(\kappa), q_{\xi}, \vec{B}\right) \leq{ }^{*} \mathbf{p}$ for some $\xi<\kappa^{+}$and some measure one sequence $\vec{B}$ on the interval $(\kappa, i(\kappa))$. The set $G^{*}$ is not generic, but any two of its elements are compatible, and if $S$ is any set of sentences of the forcing language for $\mathbf{R}_{1}$ with $|S| \leq \kappa$, then there is $\mathbf{p}^{*} \in G^{*}$ such that $\mathbf{p}^{*} \| \sigma$ for all $\sigma \in S$. Also, $(i(\kappa),(0, \gamma), \vec{B}) \in G^{*}$ for any $\gamma<i(\kappa)$ and any measure one sequence $\vec{B}$.

Define $U^{*} \supset U_{\kappa, 0}$ in $V[C]$ as follows: if $\dot{x}$ is a $\mathbf{R}_{\kappa+1}$-term for a subset $x$ of $\kappa$, then $x \in U^{*}$ if and only if there is $\mathbf{p}^{*} \in G^{*}$ such that $\mathbf{p}^{*} \Vdash \kappa \in i(\dot{x})$.

The crucial step in proving that $U^{*}$ is a normal ultrafilter on $\kappa$ is the following claim:

Claim 4.12. If $\mathbf{p} \in \mathbf{R}_{\kappa+1}$ then there is $\mathbf{p}^{*} \in G^{*}$ such that $\mathbf{p}^{\frown} \mathbf{p}^{*} \leq i(\mathbf{p})$.

Proof. Fix $\mathbf{p}=\mathbf{p}_{0} \frown(\kappa, \eta, \vec{B})$ in $\mathbf{R}_{\kappa+1}$. If we set $\mathbf{p}^{*}=(i(\kappa),(0, \gamma), i(\vec{B})) \in G^{*}$ then, using the identification of $(i(\kappa), \eta) \in P_{i(\kappa)}$ with $(i(\kappa),(0, \eta)) \in Q_{i(\kappa)}^{*}$,

$$
\begin{aligned}
\mathbf{p}^{\frown} \mathbf{p}^{*} & =\mathbf{p}_{0} \frown(\kappa, \eta, \vec{B}) \frown(i(\kappa),(0, \gamma), i(\vec{B})) \\
& \leq \mathbf{p}_{0} \frown(\gamma,(B, 0, \eta), i(\vec{B})\lceil\gamma) \frown(i(\kappa),(0, \gamma), i(\vec{B})) \\
& \leq \mathbf{p}_{0} \frown(i(\kappa),(0, \eta), i(\vec{B}))=i(\mathbf{p}),
\end{aligned}
$$

where, in order to make (13) valid, $\gamma$ is any ordinal in the interval $\kappa<\gamma<i(\kappa)$ such that

$$
\operatorname{cf}(o(\gamma))>\eta \quad \text { and } \quad \kappa \in B=i(\vec{B})_{\gamma} .
$$

In order to verify that there exists a ordinal $\gamma$ satisfying (14), set $\beta=\eta^{+}$and let $A=\left\{\nu<\kappa: \exists \gamma>\nu\left(o(\gamma)=\beta\right.\right.$ and $\left.\left.\nu \in B_{\gamma}\right)\right\}$. Then there is an ordinal $\gamma$ satisfying (14) if and only if $\kappa \in i(A)$, that is, if and only if $A \in U_{\kappa, 0}$. Now consider the canonical embedding $j: V \rightarrow M^{\prime}=\operatorname{ult}\left(V, U_{\kappa, \beta}\right)$. Since $M^{\prime} \models o(\kappa)=\beta$, we have $A=i(A) \cap \kappa \supset i(\vec{B})_{\kappa} \in U_{\kappa, 0}^{M^{\prime}}=U_{\kappa, 0}$. Thus $A \in U_{\kappa, 0}$, as required.

Now we can check that $U^{*}$ is well defined. Suppose that $\mathbf{p} \in \mathfrak{G}(C)$ and $\mathbf{p} \Vdash \dot{x}=$ $\dot{x}^{\prime}$. Then by the claim there is $\mathbf{p}^{*} \in G^{*}$ such that $i(\mathbf{p}) \geq \mathbf{p}^{\frown} \mathbf{p}^{*}$, so $\mathbf{p}^{-} \mathbf{p}^{*} \Vdash i(\dot{x})=$ $i\left(\dot{x}^{\prime}\right)$ in $M[C]$. We can assume that $\mathbf{p}^{*}$ also decides $\kappa \in i(\dot{x})$, so $\mathbf{p}^{-} \mathbf{p}^{*} \Vdash(\kappa \in i(\dot{x})$ if and only if $\left.\kappa \in i\left(\dot{x}^{\prime}\right)\right)$.

Finally, we verify that $U^{*}$ is a normal measure on $\kappa$. For any term $\dot{x}$ denoting a subset of $\kappa$ there is a condition $p^{*} \in G^{*}$ deciding both of the sentences $\kappa \in i(\dot{x})$ and $\kappa \in i(\kappa \backslash \dot{x})$, so it must decide exactly one of them affirmatively. Hence exactly one of the sets $x$ and $\kappa \backslash x$ is in $U^{*}$. Similarly, $U^{*}$ is normal (and hence $\kappa$-complete) since if $\dot{f}$ is a term denoting a function $f: \kappa \rightarrow \kappa$, then there is a condition $p^{*} \in G^{*}$ which decides all of the $\kappa$-many sentences $i(\dot{f})(\kappa)=\eta$ for $\eta<\kappa$, as well as the sentence $i(\dot{f})(\kappa) \nless \kappa$. Again, $p^{*}$ must decide exactly one of these sentences affirmatively.

This finishes the proof of the main theorem 1.1 


\section{EXtensions to these RESUlts}

In this final section generalize these results in two directions: first we consider the consistency strength of preserving other large cardinal properties between inaccessibility and measurability, and then we consider the possibility of strengthening the hypothesis that $C$ is contained in the set $D$ of inaccessible cardinals of the ground model.

Definition 5.1. $\quad$ 1. A sequence $\vec{B}$ is a full measure one sequence if $B_{\gamma} \in U(\gamma)=$ $\bigcap_{\beta<o(\gamma)} U_{\gamma, \beta}$ for all $\gamma \in D$.

2. $\mathcal{D}_{\beta}$ is the filter on $\kappa$ generated by the sets $D_{\beta}^{\vec{B}}=\{\gamma: o(\gamma) \geq \gamma\} \cup \bigcup\left\{B_{\gamma}\right.$ : $o(\gamma) \geq \beta\}$ for all full measure one sequences $\vec{B}$.

3. $\mathcal{D}=\bigcap_{\beta<\kappa} \mathcal{D}_{\beta}$.

4. $\mathcal{D}^{*}$ is the normal filter generated by the sets $D^{\vec{B}}=\triangle_{\beta} D_{\beta}^{\vec{B}}$ where $\vec{B}$ is a full measure one sequence.

Note that $\mathcal{D}^{*}$ is the smallest normal filter containing $\mathcal{D}$.

Lemma 5.2. Suppose that $C$ is a closed and unbounded subset of a cardinal $\kappa \geq \omega_{2}$ such that every $\lambda \in C$ is inaccessible in $K$, let $C^{\prime}$ be the set of inaccessible cardinals in $C$, and let $\vec{B}$ be a full measure sequence in $K$. Then $C^{\prime} \backslash\left(\beta \cup D_{\beta}^{\vec{B}}\right)$ is finite for each $\beta<\kappa$, and hence $C^{\prime} \backslash D^{\vec{B}}$ is nonstationary.

Proof. The first claim follows immediately from the proof of lemma 1.2. The second claim follows by Fodor's theorem.

Theorem 5.3. Let $\Phi(\kappa, C)$ be the formula stating that $C$ is a closed and unbounded subset of $\kappa$ containing only inaccessibles of $K$. Then we have the following equiconsistencies:

1. $\operatorname{Con}(\mathrm{ZFC}+\Phi(\kappa, C)+\kappa$ is Mahlo $)$ if and only if $\mathrm{Con}(\mathrm{ZFC}+$ every set in $\mathcal{D}^{*}$ is stationary).

2. $\operatorname{Con}(\mathrm{ZFC}+\Phi(\kappa, C)+\kappa$ is weakly compact) if and only if $\mathrm{Con}(\mathrm{ZFC}+n o$ set in $\mathcal{D}$ is in the weakly compact ideal).

3. $\mathrm{Con}(\mathrm{ZFC}+\Phi(\kappa, C)+\kappa$ is Ramsey) if and only if $\mathrm{Con}(\mathrm{ZFC}+\kappa \rightarrow$ $\left.\left(\mathcal{D}^{+}\right)_{2}^{<\omega}\right)$, where $\mathcal{D}^{+}$is the set of sets $y \subset \kappa$ such that $\kappa \backslash y \notin \mathcal{D}$.

Proof of necessity. In order to show that the stated conditions are necessary, we will show that if the property on the left holds, there is no inner model with a Woodin cardinal, and $K$ is Steel's core model then the property on the right holds in $K$. Note that we are proving slightly more than the equiconsistency asserted in the theorem, for which it would be sufficient to assume that there is no inner model with $o(\kappa)=\kappa$.

Necessity for Mahlo cardinals. Let $E^{V}$ be the set of inaccessible cardinals of $V$. Then $E^{V}$ is stationary in $\kappa$ since $\kappa$ is Mahlo, and if $D \in \mathcal{D}^{*}$ then $E^{V} \backslash D$ is nonstationary in $V$ by lemma 5.2 Thus $D$ is stationary in $V$, and hence is stationary in $K$.

Necessity for weakly compact cardinals. Suppose that $D \in \mathcal{D}^{*}$, and let a $\Pi_{1}^{1}$ formula $\phi$ and a set $R \subset \kappa^{2}$ in $K$ be given such that $(\kappa, R) \models \forall A \phi(A)$ holds in $K$. We need to show that there is a cardinal $\lambda \in D$ of $K$ such that $\left(\lambda, R \cap \lambda^{2}\right) \models \forall A \phi(A)$ holds in $K$. 
By lemma 5.2 there is a closed unbounded subset $C_{1} \subset C$ such that every inaccessible member of $C_{1}$ is in $D$. If we let $\mathcal{A}^{\prime}$ be the structure $\left(\kappa, R, C_{1}\right)$ in $V$, then $\mathcal{A}^{\prime} \models\left(C_{1}\right.$ is unbounded and $\left.\forall A \subset \kappa(A \in K \Longrightarrow \phi(A))\right)$. Since " $A \in K$ " can be expressed by a $\Sigma_{1}^{1}$ formula over $V_{\kappa}$, this formula is $\Pi_{1}^{1}$ and since $\kappa$ is weakly compact in $V$ it follows that there is an inaccessible cardinal $\lambda<\kappa$ in $V$ such that $\left(\lambda, R \cap \lambda^{2}, C_{1} \cap \lambda\right) \models\left(C_{1} \cap \lambda\right.$ is unbounded and $\left.\forall A \subset \kappa(A \in K \Longrightarrow \phi(A))\right)$.

Finally, $\lambda \in D$ since $\lambda$ is in $C_{1}$ and $\lambda$ is inaccessible in $V$, and in $K$ we have $\left(\lambda, R \cap \lambda^{2}\right) \models \forall A \phi(A)$ as required.

Necessity for Ramsey cardinals. We need the following lemma:

Lemma 5.4. Assume that $\kappa$ is Ramsey in $V$ and that $C \subset \kappa$ is closed and unbounded. Then for any function $f:[\kappa]^{<\omega} \rightarrow 2$ in $K$ there is a set $D \in[\kappa]^{\kappa}$ in $K$ which is homogeneous for $f$ such that either $o(\nu)=\nu$ for all $\nu \in D$ or else the set of cardinals $\nu \in D \cap C$ which are inaccessible in $V$ is unbounded.

Sketch of proof. For the sake of brevity we simply outline the modifications of the proof of the main theorem of [6] which are required to prove lemma 5.4. We assume that the reader of this sketch is familiar with that paper and has a copy of it available.

Let $I_{0}$ be a set of indiscernibles for the structure $\mathcal{S}=\left(V_{\kappa}, f, C\right)$ such that $\left|I_{0}\right|=\kappa$ and the $\omega^{\text {th }}$ member of $I_{0}$ is as small as possible. Thus $I_{0}$ is normal for functions definable in $\mathcal{S}$, and in particular $I_{0} \subset C$. Use $I_{0}$ to define an elementary substructure of $V_{\kappa}$, and proceed with the construction of [6], noting that $\delta$ of that paper is equal to $\kappa$, since we are dealing with a Ramsey cardinal.

Now we modify the definition of the set $I$ in corollary 3.10 in [6]: first, take $I_{1}$ to be the set of indiscernibles for $\kappa$ from the tree $\mathcal{T}$ : thus $\nu \in I_{1}$ if and only if there is $\xi$ on the main branch of $\mathcal{T}$ such that $\nu=\operatorname{crit}\left(i_{\xi, \kappa}\right)$ and $i_{\xi, \kappa}(\nu)=\kappa$. The normality of $I_{0}$ can be used to show that $j_{0, \theta}$ " $I_{0} \subset I_{1}$. The normality of $I_{0}$ also implies that either $o(\nu)>\nu$ for all $\nu \in I_{0}, o(\nu)=\nu$ for all $\nu \in I_{0}$, or there is some $\gamma_{0}<\kappa$ such that $I_{0} o(\nu)=\gamma_{0}$ for all $\nu \in I_{0}$. If $o(\nu)>\nu$ for $\nu \in I_{0}$, we use the observation in the 2nd paragraph before corollary 3.10 of [6]: The final model $\mathcal{M}_{\delta}$ of the tree $\mathcal{T}$ contains, as a member, a measure of order $\kappa$ on $\kappa$. Thus $\mathcal{M}_{\delta}$ contains, as a member, a set $\bar{D}$ of indiscernibles for $j_{\theta}(f)$. Since ultrapowers of initial segments of $\mathcal{M}_{\delta}$ are well founded, this can be used to define a set $D$ of indiscernibles in $K$ satisfying the first alternative in the statement of lemma 5.4 .

For the other two cases, modify the definition of $I$ in corollary 3.10 to be the set of $\nu \in I_{1}$ such that $o(\nu)=\gamma_{0}$ or $o(\nu)=\nu$, depending on the value of $o(\nu)$ for $\nu \in I_{0}$. The relation $\epsilon_{0}$ used throughout the rest of the proof should be replaced by $\epsilon_{\gamma_{0}}$ or $\epsilon_{\nu}$, where $\nu \in_{\gamma} X$ if and only if $\nu \in X$ and $o(\nu)=\gamma$. Note that our elimination of the case $o(\nu)>\nu$ allows us to skip the second case, $x \in U_{\nu, 0}$ if $o(\nu)>0$, of the definition of $\epsilon_{0}$ in [6]. This simplification could have also been made in [6].

The set $I$ defined in corollary 1.10 of [6] is closed and unbounded, while our set $I$ need not be stationary. A check of the rest of the proof shows that this does not affect the proof: the only use of stationarity is in the first paragraph of the proof of lemma 3.12 and that proof actually uses $I_{1}$ rather than $I$. The set $D \in K$ of indiscernibles for $f$ which is given by this construction will contain the original set $I_{0}$, and since every member of that set is inaccessible in $V$ it satisfies the second alternative of lemma 5.4 This completes the proof of lemma 5.4 . 
Now suppose that $\kappa$ is Ramsey in $V$, let $f:[\kappa]^{<\omega} \rightarrow 2$ be a function in $K$ and let $D \in K$ be the set of indiscernibles for $f$ given by lemma 5.4 using the set $C$ from theorem 5.3 If $o(\nu)=\nu$ for all $\nu \in D$ then $D \in \mathcal{D}_{\beta}^{\vec{B}}$ for any sequence $\vec{B}$ and ordinal $\beta$, so we can assume the second alternative of the lemma: that the set of $\nu \in D \cap C$ which are inaccessible in $V$ is unbounded in $\kappa$. Then for any full measure one sequence $\vec{B}$ and any $\beta<\kappa$, lemma 5.2 implies that the set of inaccessible cardinals in $C \backslash D_{\beta}^{\vec{B}}$ is bounded, and hence $D \cap D_{\beta}^{\vec{B}}$ is unbounded in $\kappa$. Thus $D \in \mathcal{D}^{+}$.

This completes the proof of necessity for all three clauses of theorem 5.3

Proof of sufficiency. In order to prove the sufficiency of the condition on the right in each clause of theorem 5.3 we show that if that condition holds in $V$ then the condition on the left holds in $V[C]$, where $C$ is generic for the forcing $\mathbf{R}_{\kappa+1}$ used in the proof of the main theorem (or, for a Ramsey cardinal, for a slight modification of the forcing $\mathbf{R}_{\kappa+1}$ ). First we introduce some notation.

If $\mathbf{p}=(p, \vec{B}) \in \mathbf{R}_{\kappa+1}$ and $\lambda \in D^{\vec{B}} \backslash\lceil p\lceil\kappa\rceil$, then let add(p, $\lambda)$ be the following condition, which uses either $P_{\lambda}$ or $Q_{\lambda}^{*}$ to add $\lambda$ to the domain of $p$ :

$$
\operatorname{add}(\mathbf{p}, \lambda)= \begin{cases}\mathbf{p}\left\lceil\kappa ^ { \frown } \left(\lambda, \kappa_{p}, \vec{B}\lceil\lambda) \frown\left(\kappa, \kappa^{\prime}, \vec{B} \mid \kappa^{\prime}\right)\right.\right. & \text { if } o(\lambda)<\lambda, \\ \mathbf{p} \mid \kappa^{-}\left(\lambda,\left(0, \kappa_{p}\right), \vec{B}\lceil\lambda) \frown(\kappa, \lambda, \vec{B} \mid \lambda)\right. & \text { if } o(\lambda) \geq \lambda,\end{cases}
$$

where we write $\mathbf{p}=\mathbf{p}\left\lceil\kappa^{-}\left(\kappa, \kappa_{p}, \vec{B}\right)\right.$, and if $o(\lambda)<\lambda$ then $\kappa^{\prime}$ is the least ordinal such that for all $\beta<\lambda$ there is a $\gamma<\kappa^{\prime}$ with $\operatorname{cf}(o(\gamma))>\beta$ such that $\lambda \in B_{\gamma}$.

Note that if $\lambda \in D^{\vec{B}} \backslash \kappa_{p}$, then add $(\mathbf{p}, \lambda) \leq \mathbf{p}$, and (except for the choice of $\kappa^{\prime}$ ) $\operatorname{add}(\mathbf{p}, \lambda)$ is the weakest extension of $\mathbf{p}$ containing an instance of $P_{\lambda}$. Furthermore if $\mathbf{p}^{\prime} \leq \mathbf{p}$ or $\mathbf{p}^{\prime} \leq{ }^{*} \mathbf{p}$ and $\mathbf{p}^{\prime}$ agrees with $\mathbf{p}$ above $\lambda$ then $\operatorname{add}\left(\mathbf{p}^{\prime}, \lambda\right) \leq \operatorname{add}(\mathbf{p}, \lambda)$ or $\operatorname{add}\left(\mathbf{p}^{\prime}, \lambda\right) \leq^{*} \operatorname{add}(\mathbf{p}, \lambda)$, respectively.

Sufficiency for Mahlo cardinals. Let $\dot{C}_{1}$ be a name for a closed and unbounded subset of $\kappa$, and let $\mathbf{p}=(p, \vec{B}) \in \mathbf{R}_{\kappa+1}$ be arbitrary. We will find a cardinal $\lambda<\kappa$ and a condition $\mathbf{p}^{\prime} \leq \mathbf{p}$ so that $\mathbf{p}^{\prime}$ forces that $\lambda$ is an inaccessible limit point of $\dot{C}_{1}$. For each $\alpha<\kappa$ pick a condition $\mathbf{p}_{\alpha}=\left(p_{\alpha}, \vec{B}_{\alpha}\right) \leq * \mathbf{p}\left\lfloor\alpha\right.$ in $\mathbf{R}_{\alpha, \kappa+1}$ so that there is $\eta_{\alpha}<\kappa$ such that $\mathbf{p}_{\alpha} \Vdash \inf \left(\dot{C}_{1} \backslash \alpha\right)<\eta_{\alpha}$. Let $\vec{B}^{\prime}$ be the diagonal intersection of the sequences $\vec{B}^{\alpha}$, and write $\kappa_{\alpha}$ for $\kappa_{p^{\alpha}}$, so that $\mathbf{p}_{\alpha}=\left(p_{\alpha}\left\lceil\kappa_{\alpha}, \vec{B}_{\alpha}\left\lceil\kappa_{\alpha}\right) \frown\left(\kappa, \kappa_{\alpha}, \vec{B}_{\alpha} \mid \kappa_{\alpha}\right)\right.\right.$.

Since $D^{\vec{B}^{\prime}}$ is stationary by hypothesis, there is a cardinal $\lambda \in D^{\vec{B}^{\prime}}$ such that $\bar{\kappa}<\lambda$ and $\eta_{\alpha}, \kappa_{\alpha}<\lambda$ for each $\alpha<\lambda$. Set $\mathbf{p}^{\prime}=\operatorname{add}\left(\left(p, \vec{B}^{\prime}\right), \lambda\right)$. Then $\mathbf{p}^{\prime} \leq \mathbf{p}$ and $\mathbf{p}^{\prime}$ forces that $\lambda$ is inaccessible in $V[\dot{C}]$.

I claim that $p^{\prime}$ forces that $\lambda$ is a limit point of $\dot{C}_{1}$, and hence is a member of $\dot{C}_{1}$. Suppose to the contrary that there is a condition $\mathbf{p}^{\prime \prime} \leq \mathbf{p}^{\prime}$ so that $\mathbf{p}^{\prime \prime} \Vdash \dot{C}_{1} \cap \lambda \subset \alpha$ for some $\alpha<\lambda$. By increasing $\alpha$ if necessary, we can assume that $\alpha \geq\left\lceil p^{\prime \prime}\lceil\lambda\rceil\right.$. Since $\mathbf{p}_{\alpha} \leq^{*} \mathbf{p}$, and since $\left\lceil\mathbf{p}^{\prime \prime}\lceil\lambda\rceil<\left\lfloor\mathbf{p}_{\alpha}\right\rfloor<\kappa_{\alpha}<\lambda\right.$ there is a $\mathbf{p}_{\alpha}^{\prime} \leq^{*} \mathbf{p}_{\alpha}$, with the same domain as $\mathbf{p}_{\alpha}$, so that

$$
\mathbf{p}^{\prime \prime \prime}=\mathbf{p}^{\prime \prime} \mid \lambda \frown \mathbf{p}_{\alpha}^{\prime}\lceil\overbrace{}^{-}\left(\lambda, \kappa_{\alpha}, \vec{B}_{\alpha}\right) \frown \mathbf{p}^{\prime \prime} \mid \lambda \leq \mathbf{p}^{\prime \prime} .
$$

Also, $\mathbf{p}^{\prime \prime \prime} \leq \mathbf{p}_{\alpha}$, since by the definition of $\operatorname{add}(\mathbf{p}, \lambda)$ the ordinal $\kappa^{\prime}=\kappa_{\mathbf{p}^{\prime}}$ is large enough that $\lambda \in D_{\beta}^{\vec{B}^{\prime}\left\lceil\kappa^{\prime}\right.}$ where $\beta=\left\lceil\mathbf{p}_{\alpha}\lceil\lambda\rceil\right.$. It follows that $\mathbf{p}^{\prime \prime \prime} \Vdash \inf \left(\dot{C}_{1} \backslash \alpha\right) \leq$ $\eta_{\alpha}<\lambda$, contrary to the choice of $\alpha$. Thus $\lambda$ is a limit point of $C_{1}$. 
Sufficiency for weakly compact cardinals. We need introduce some further notation before showing that $\kappa$ has the tree property in $V[C]$. If $\mathbf{p} \in \mathbf{R}_{\kappa+1}$ and $\mathbf{p}=$ $\mathbf{p} \mid \kappa^{\mathbf{p}} \frown\left(\kappa, \kappa^{\mathbf{p}}, \vec{B}\right)$ then we write $L(\mathbf{p})$ for $\left(\mathbf{p}\left\lceil\kappa^{\mathbf{p}}, \kappa^{\mathbf{p}}\right)\right.$, which we call the localization of p. We write $\tau^{\vec{B}}$ for the converse operation: if $\tau=(\mathbf{p}, \eta)$ then $\tau^{\vec{B}}=\mathbf{p} \frown(\kappa, \eta, \vec{B} \mid \eta)$.

Let $T$ be an arbitrary tree in $V[C]$ such that each level and each branch of $T$ has size less than $\kappa$. We will show that $T$ has height less that $\kappa$. We can assume that the universe of $T$ is contained in $\kappa$. Let $C_{T}=\left\{\nu: T_{\nu} \subset \nu\right\}$, where $T_{\nu}$ is the set of nodes of $T$ of height less than $\nu$. Then there is a measure one sequence $\vec{B}=\vec{B}^{T}$ such that if $\nu<\nu^{\prime}<\kappa_{p}$ then $\mathbf{p} \Vdash \nu<^{T} \nu^{\prime}$ if and only if $L(p)^{\vec{B}} \Vdash \nu<^{T} \nu^{\prime}$, and $p \Vdash \nu \in C_{T}$ if and only if $L(\mathbf{p})^{\vec{B}} \Vdash \nu \in C_{T}$. We will write $\tilde{T}$ for $\left\{\left(\nu, \nu^{\prime}, L(\mathbf{p})\right): \mathbf{p} \Vdash \nu^{\prime}<\nu\right\}$, and $\tilde{C}_{T}$ for $\left\{(\nu, L(\mathbf{p})): \mathbf{p} \Vdash \nu \in C_{T}\right\}$.

We will represent arbitrary subsets of $\kappa$ similarly: we say that a pair $\left(\tilde{A}, \vec{B}^{A}\right)$ represents the set $A=\left\{\nu: \exists(\nu, \tau) \in \tilde{A}\left(\tau^{\vec{B}^{A}} \in G\right)\right\}$ where $G=\mathfrak{G}(C)$. Notice that for any subset $A$ of $\kappa$ there is a pair $\left(\tilde{A}, \vec{B}^{A}\right)$ which represents $A$, and furthermore the subsets of $\kappa$ which are contained in a branch of $T$ are exactly those sets $A$ which can be represented by a pair $\left(\tilde{A}, \vec{B}^{A}\right)$ which satisfy the following first order conditions over the structure $\left(V_{\kappa}, \tilde{A}, \vec{B}^{A}, \tilde{T}, \tilde{C}, \vec{B}\right)$ :

1. $\vec{B}^{A} \subset \vec{B}^{T}$.

2. $\forall\left(\nu, \tau_{0}\right) \in \tilde{A} \forall \tau_{1}\left(\tau_{1}^{\vec{B}^{A}} \leq \tau_{0}^{\vec{B}^{A}} \Longrightarrow\left(\nu, \tau_{1}\right) \in \tilde{A}\right)$.

3. $\forall \nu<\nu^{\prime}<\kappa \forall \tau\left((\nu, \tau) \in \tilde{A}\right.$ and $\left.\left(\nu^{\prime}, \tau\right) \in \tilde{A} \Longrightarrow\left(\nu, \nu^{\prime}, \tau\right) \in \tilde{T}\right)$.

We need a $\Pi_{1}^{1}$ formula in $V$ which asserts that $\Vdash \dot{T}$ has no branches of length $\kappa$. The formula will assert that no pair $\left(\tilde{A}, \vec{B}^{A}\right)$ represents a subset of a branch of size $\kappa$, so we need to verify that the assertion that " $p_{0}$ forces that $A=\{\nu: \exists(\nu, \tau) \in \tilde{A}$ : $\left.\tau^{\vec{B}^{A}} \in G\right\}$ is bounded in $\kappa$ " can be stated by a first order formula over $V_{\kappa}$ which will have the same meaning over $V_{\lambda}$ for any $\lambda \in D^{\vec{B}^{T}}$. Note that we can assume that every such $\lambda$ is weakly compact.

We state the following claim for arbitrary weakly compact $\lambda$, with the same notation as for $\kappa$. If $Y \subset \mathbf{R}_{\lambda+1}$ then we write $Y_{-}$for $\{p: p \Vdash G \cap Y=\varnothing\}$.

Claim 5.5. Suppose $\lambda$ is weakly compact in $V$, and that $\left(X_{\nu}: \nu<\lambda\right)$ is a sequence of subsets of $\mathbf{R}_{\lambda+1}$ such that $L\left[X_{\nu}\right]=\left\{L(p): p \in X_{\nu}\right\}$ is uniformly first order definable over $V_{\lambda}$. Then $L\left[\left(\bigcup_{\nu<\lambda} X_{\nu}\right)_{-}\right]$is first order definable over $V_{\lambda}$.

Proof of claim. In order to determine whether an given localization $\tau$ is in $L\left[\left(\bigcup_{\nu<\lambda} X_{\nu}\right)_{-}\right]$, we define a tree $\mathcal{S}_{\tau}$. A node of $\mathcal{S}_{\tau}$ of height $\eta<\lambda$ is a measure one sequence $\vec{Y}$ defined on the interval $(0, \eta)$, such that for no $\nu<\eta$ is there a condition $\mathbf{p} \in X_{\nu}$ with $L(p) \in V_{\eta}$ and $\mathbf{p} \leq \tau^{\vec{Y}} \vec{B} \mid \eta$. Note that $\mathcal{S}$ is definable from the sequence of sets $L\left[X_{\nu}\right]$ for $\nu<\lambda$. Furthermore a condition $\mathbf{p}$ with $L(\mathbf{p})=\tau$ such that $\mathbf{p} \Vdash G \cap \bigcup_{\nu<\lambda} X_{\nu}=\varnothing$ corresponds to an unbounded branch $\left(\vec{B} \mathbf{p}^{\mathbf{p}}\lceil\eta: \eta<\kappa)\right.$ through $\mathcal{S}_{\tau}$. Thus $\tau$ is in $L\left[\left(\bigcup_{\nu<\lambda} X_{\nu}\right)_{-}\right]$if and only if $\mathcal{S}_{\tau}$ has an unbounded branch, and since $\lambda$ is weakly compact this holds iff the tree $\mathcal{S}_{\tau}$ has height $\lambda$, which is a first order statement about $\tau$ in $V_{\lambda}$.

Now set $X_{\nu}=\{\tau:(\nu, \tau) \in \tilde{A}\}$. Then $Y_{\nu}=L\left[\left(\bigcup_{\nu^{\prime}>\nu} X_{\nu^{\prime}}\right)_{-}\right]$is the set of localizations $L(p)$ such that $p \Vdash \dot{A} \subset \nu$, and $Y=L\left[\left(\bigcup_{\nu} Y_{\nu}\right)_{-}\right]$is the set of localizations $L(p)$ such that $p \Vdash \dot{A}$ is unbounded. Thus $Y$ is first order definable, but $Y$ is empty if and 
only if $\Vdash \sup (\dot{A})<\lambda$. Thus there is a $\Pi_{1}^{1}$ formula $\Phi$ such that $\left(V_{\kappa}, \tilde{T}, \tilde{C}, \vec{B}^{T}\right) \models \Phi$ if and only if $\Vdash \dot{T}$ has no unbounded branches.

Now, since $D^{\vec{B}^{T}}$ is in the weakly compact filter in $V$, there is $\lambda \in D^{\vec{B}^{T}}$ such that

$$
\begin{gathered}
\left(\lambda, \tilde{T} \cap V_{\lambda}, \vec{B}^{T}\left\lceil\lambda, \tilde{C} \cap V_{\lambda}\right) \prec(\kappa, \tilde{T}, \vec{B}, \tilde{C}),\right. \\
\left(\lambda, \tilde{T} \cap V_{\lambda}, \vec{B}\left\lceil\lambda, \tilde{C} \cap V_{\lambda}\right) \models \Phi .\right.
\end{gathered}
$$

Let $\mathbf{p}^{\prime}=\operatorname{add}(\mathbf{p}, \lambda)$, and for arbitrary $\tau$ write $\tau^{\vec{B}, \lambda}=\operatorname{add}\left(\tau^{\vec{B}}, \lambda\right)$. Then $\tau^{\vec{B}, \lambda} \leq \mathbf{p}^{\prime}$, and $\tau_{0} \leq^{\vec{B}} \tau_{1}$ iff $\tau_{0}^{\vec{B}, \lambda} \leq \tau_{1}^{\vec{B}, \lambda}$. It follows that $\mathbf{p}^{\prime}$ forces that $T_{\lambda}$ and $C_{T}$ are represented by $\tilde{T} \cap V_{\lambda}, \tilde{C} \cap V_{\lambda}$, and $\vec{B}\left\lceil\lambda\right.$ just as $T$ and $C_{T}$ are represented by $\tilde{T}$ and $\vec{B}$. In particular, (15a) implies that $T_{\lambda} \subset \lambda$. Finally, (15b) implies that $T_{\lambda}$ has no branches of length $\lambda$. It follows that $T$ has no branches of length $\lambda$, so $T$ has height at most $\lambda$.

Sufficiency for Ramsey cardinals. For this proof we need to modify the forcing $\mathbf{R}_{\kappa+1}$ slightly by adding a new recipe, $Q_{\gamma}^{0}$, which is defined only for measurable cardinals $\gamma$ with $o(\gamma)=1$. An instance of this recipe is indicated by a triple $(\gamma, A, \vec{f})$ where $A \in U_{\gamma}$ and $\vec{f}$ is a sequence of functions $f_{n}$ with domain $A$ such that if $\eta \in A$ then $f_{n}(\eta)$ is an $\eta$-closed condition in $\mathbf{R}_{\eta, \gamma}$. The closed set $C_{p}$ of $\gamma$ added by a condition $p=(\gamma, A, \vec{f})$ has the form $\bigcup_{n<\omega} C_{f_{n}\left(\alpha_{n}\right)}$ where $\left\{\alpha_{n}: n<\omega\right\}$ is a Prikry sequence in $\gamma$. A basic non-direct extension (corresponding to clause 3 of definition 2.12) has the form

$$
f_{0}(\alpha) \frown\left(\gamma, A \backslash\left\lceil f_{0}(\alpha)\right\rceil,\left\langle f_{n}: 1 \leq n<\omega\right\rangle\right) \leq(\gamma, A, \vec{f}) .
$$

Note one significant difference between $Q_{\gamma}^{0}$ and $Q_{\gamma}$ : the former does not include the Prikry sequence in $C$, while the latter does. Note that a condition of the form $\mathbf{p} \frown(\gamma, A, \vec{f}) \frown(\lambda, \bar{\lambda})$ forces that for each $n$ the generic set $\mathfrak{G}(C)$ contains a condition $\mathbf{p}^{\frown} f_{0}\left(\gamma_{0}\right) \frown \ldots \frown f_{n}\left(\gamma_{n}\right) \frown\left(\lambda,\left\lceil f_{n}\left(\gamma_{n}\right)\right\rceil\right)$.

Let $C \subset \kappa$ be generic for this modified version of $\mathbf{R}_{\kappa+1}$, and suppose $\dot{h}$ is a name for a function $h:[\kappa]^{<\omega} \rightarrow 2$ in $V[C]$. We will find a set $I^{*} \in[\kappa]^{\kappa}$ in $V[C]$ which is homogeneous for $h$.

Fix $\vec{B}^{0}$ so that if $\nu<\lceil p\rceil$ and $p \Vdash \dot{h}(\vec{\nu})=i$ then $L(p)^{\vec{B}^{0}} \Vdash \dot{h}(\vec{\nu})=i$, and define the function $\tilde{h}$ by $\tilde{h}(\tau, \vec{\nu})=i$ if $\tau^{\vec{B}^{0}} \Vdash h(\vec{\nu})=i$. Now let $I \in \mathcal{D}^{+}$be a set of indiscernibles in $V$ for the structure $\mathcal{S}=\left(V_{\kappa}, \tilde{h}, \vec{B}^{0}\right)$, using, as in the proof of lemma 5.4, Silver's trick of picking the set $I$ with the smallest possible $\omega^{\text {th }}$ member so that $I$ is normal in the following sense: if $\lambda \in[I]^{n}, x \in V_{\lambda_{0}}$, and $\tau(x, \vec{\lambda})<\lambda_{0}$ where $\tau$ is any function which is first order definable (without parameters) in $\mathcal{S}$, then $\tau\left(x, \vec{\lambda}^{\prime}\right)=\tau(x, \vec{\lambda})$ for every $\vec{\lambda}^{\prime} \in\left[I \backslash \lambda_{0}\right]^{n}$.

We will define a measure one sequence $\vec{B}^{\omega} \subset \vec{B}^{0}$, along with conditions $t_{\gamma}$ for $\gamma$ in a cofinal set $T \subset \kappa$, with the following property: Define $\mathbf{p}_{k}(\bar{\lambda}, \vec{\lambda}, \vec{\gamma})$ to be the condition

$$
\begin{aligned}
\left(\lambda_{k}, \bar{\lambda}, \vec{B}^{\omega}\right) \frown t_{\gamma_{k}} \frown\left(\lambda_{k-1}, \gamma_{k}, \vec{B}^{\omega}\right) \frown t_{\gamma_{k-1}} \frown & \cdots \\
& \frown\left(\lambda_{1}, \gamma_{2}, \vec{B}^{\omega}\right) \frown t_{\gamma_{1}} \frown\left(\kappa, \gamma_{1}, \vec{B}^{\omega}\right)
\end{aligned}
$$

for any sequence $\vec{\lambda} \in[I]^{k}$ and $\vec{\gamma} \in[T]^{k}$ such that

$$
\lambda_{k}<\gamma_{k}<\lambda_{k-1}<\gamma_{k-1}<\cdots<\lambda_{1}<\gamma_{1}<\kappa .
$$


Then for each $k \in \omega$ there is a set $X \subset \mathbf{P}_{\kappa}$ of conditions such that (i) for each $\lambda \in I$, the set of conditions $\mathbf{p} \cap(\lambda, \bar{\lambda}, \vec{B})$ with $\mathbf{p} \in X$ is dense below $\left(0, \lambda, \vec{B}^{\omega}\right)$, and (ii) if $\mathbf{p} \in X$ then there is some $i \in\{0,1\}$ such that $\mathbf{p}^{\frown} \mathbf{p}_{k}(\lceil\mathbf{p}\rceil, \vec{\lambda}, \vec{\gamma}) \Vdash \dot{h}(\vec{\lambda})=\check{\imath}$ for any $\vec{\lambda} \in[I \backslash\lceil\mathbf{p}\rceil]^{k}$ and $\vec{\gamma} \in[T]^{k}$ satisfying (17).

Assume that we have defined the sequence $\vec{B}^{\omega}$ and conditions $t_{\gamma}$, and define $I^{*}$ to be the set of cardinals $\lambda \in I$ such that $\mathfrak{G}(C)$ contains a condition including a sequence $\left(\lambda, \bar{\lambda}, \vec{B}^{\omega}\right) \frown t_{\gamma}$ for some $\bar{\lambda}<\lambda$ and $\gamma \in T \backslash \lambda+1$. The following claim then completes, modulo the construction of $\vec{B}^{\omega}$ and $t_{\gamma}$, the proof of sufficiency for Ramsey cardinals:

Claim 5.6. The set $I^{*}$ is a set of cardinality $\kappa$ which is homogeneous for $h$.

Proof of claim. To see that $I^{*}$ is homogeneous, notice that if $\vec{\lambda} \in\left[I^{*}\right]^{k}$ then there is a witnessing sequence $\vec{\gamma}$ and a condition $\mathbf{p} \in \mathbf{R}_{\lambda_{k}}$ such that $\mathbf{p}^{-} \mathbf{p}_{k}(\vec{\lambda}, \vec{\gamma}) \in \mathfrak{G}(C)$ and $\mathbf{p}^{\frown} \mathbf{p}_{k}(\vec{\lambda}, \vec{\gamma})$ forces $h(\vec{\lambda})=i$ for some $i \in\{0,1\}$. Then any other sequence $\vec{\lambda}^{\prime} \in\left[I^{*}\right]^{k}$ also has a witnessing sequence $\vec{\gamma}^{\prime} \in[T]^{k}$ such that $\mathbf{p}^{-} \mathbf{p}_{k}\left(\vec{\lambda}^{\prime}, \vec{\gamma}^{\prime}\right) \in \mathfrak{G}(C)$, and $\mathbf{p}^{\frown} \mathbf{p}_{k}\left(\vec{\lambda}^{\prime}, \vec{\gamma}^{\prime}\right)$ also forces $\dot{h}\left(\vec{\lambda}^{\prime}\right)=\check{\imath}$.

To see that $\left|I^{*}\right|=\kappa$, let $\mathbf{p}=\mathbf{p}_{0} \frown(\kappa, \bar{\kappa}, \vec{B}) \leq \mathbf{p}_{0} \frown\left(\kappa,\left\lceil\mathbf{p}_{0}\right\rceil, \vec{B}^{0}\right)$ be arbitrary, and suppose $\bar{\kappa}<\alpha<\kappa$. Since $I \in \mathcal{D}^{+}$, we can find a cardinal $\lambda \in I \cap D_{\alpha}^{\vec{B}}$, and since $T$ is unbounded in $\kappa$ we can find a cardinal $\gamma \in T \backslash(\lambda+1)$. Then, using lemma 4.3, we can find a condition $t_{\gamma}^{\prime} \leq^{*} t_{\gamma}$ so that $\mathbf{p}^{\prime}=\mathbf{p}_{0} \frown\left(\lambda, \alpha, \vec{B}^{\omega}\right) \frown t_{\gamma}^{\prime} \frown(\kappa, \gamma, \vec{B}) \leq \mathbf{p}$. Then $\mathbf{p}^{\prime} \Vdash \lambda \in I^{*} \backslash \alpha$, and since $\alpha$ and $\mathbf{p}^{\prime}$ were arbitrary it follows that $I^{*}$ is unbounded in $\kappa$.

We construct $t_{\gamma}$ and $\vec{B}^{\omega}$ out of simpler components, working down towards the bottom level where the components are definable in the structure $\mathcal{S}$.

First, $t_{\gamma}=(\gamma, A, \vec{f})$ is an instance of the recipe $Q_{\gamma}^{0}$, where

$$
\begin{aligned}
T & =\{\gamma: o(\gamma)=1 \text { and } \gamma=\bigcup(I \cap \gamma)\}, \\
f_{n}(\nu) & =\tau_{n}(\inf (I \backslash \nu)), \\
A & =\left\{\nu<\gamma: o(\nu)=0 \text { and } \bigcup_{n} f_{n} " \nu \subset V_{\nu} \text { and } \nu=\bigcup(I \cap \nu)\right\} .
\end{aligned}
$$

Notice that the hypothesis $I \in \mathcal{D}^{+}$implies that $T$ is cofinal in $\kappa$.

Down one more level, set

$$
\begin{aligned}
\tau_{n}(\lambda) & =\bigwedge_{n \leq s<\omega} \tau_{n}^{s}(\lambda), \\
\vec{B}^{\omega} & =\bigcap_{n<\omega} \vec{B}^{n} .
\end{aligned}
$$

Here $\left\langle\tau_{n}^{i}(\lambda): n \leq i<\omega\right\rangle$ will be a $\leq^{*}$-descending sequence of $\lambda$-closed conditions in $\mathbf{R}_{\lambda, \kappa}$.

This is the bottom level, and the conditions $\overrightarrow{\mathbf{p}}_{k}(\vec{\lambda}, \vec{\lambda}, \vec{\gamma})$ of (16) derive their properties from the components $\tau_{i}^{n}(\lambda)$ and $\vec{B}^{n}$. To make this more precise, write $\tau^{n}\left(\vec{\lambda}^{n}\right)=\tau_{1}^{n}\left(\lambda_{1}^{n}\right) \frown \ldots \frown \tau_{n}^{n}\left(\lambda_{n}^{n}\right)$ and

$$
\begin{aligned}
\mathbf{p}^{n}\left(\bar{\lambda}, \vec{\lambda}^{n}, \ldots, \vec{\lambda}^{1}\right)=\left(\lambda_{0}^{n}, \bar{\lambda}, \vec{B}^{n}\right) \frown \tau^{n}\left(\vec{\lambda}^{n}\right) \frown \\
\ldots \frown\left(\lambda_{0}^{1},\left\lceil\tau^{2}\left(\vec{\lambda}^{2}\right)\right\rceil, \vec{B}^{n}\right) \frown \tau^{1}\left(\vec{\lambda}^{1}\right) \frown\left(\kappa,\left\lceil\tau^{1}\left(\vec{\lambda}^{1}\right)\right\rceil, \vec{B}^{0}\right),
\end{aligned}
$$


where $\left\langle\lambda_{i}^{m}: 0 \leq i \leq m \leq n\right\rangle$ is any sequence in $[I]^{n(n+3) / 2}$ such that

$$
\lambda_{0}^{n}<\cdots<\lambda_{n}^{n}<\lambda_{0}^{n-1}<\cdots<\lambda_{0}^{1}<\lambda_{1}^{1}<\kappa .
$$

Notice that the condition $\mathbf{p}_{k}(\bar{\lambda}, \vec{\lambda}, \vec{\gamma})$ forces that $\mathbf{p}^{k}\left(\bar{\lambda}, \vec{\lambda}^{k}, \ldots, \vec{\lambda}^{1}\right) \in \mathfrak{G}(C)$ for some sequences $\vec{\lambda}^{n}, \ldots, \vec{\lambda}^{1}$ satisfying (18) such that $\lambda_{m}=\lambda_{0}^{m}$ for each $1 \leq m \leq k$.

Also notice that the fact that $\mathbf{p}^{n}$ is definable in $\mathcal{S}$, together with the fact that the sequence of parameters is from $I$, automatically implies that their properties are independent of the choice of $\vec{\lambda}^{n}, \ldots, \vec{\lambda}^{1}$. Hence the derived properties of $\mathbf{p}_{k}(\vec{\lambda}, \vec{\lambda}, \vec{\gamma})$ are independent of the choice of $\vec{\lambda}$ and $\vec{\gamma}$.

In order to define the function $\mathbf{p}^{n}$ by recursion on $n$, we actually require a stronger property than the property needed for $\mathbf{p}_{\kappa}(\bar{\lambda}, \vec{\lambda}, \vec{\gamma})$ :

We will say that a condition $\mathbf{p}$ fully decides $h$ for $\vec{\lambda}$ if, setting $\eta=\lfloor\mathbf{p}\rfloor$, one of the following two conditions is satisfied for each $\vec{\nu} \in[\eta]^{<\omega}$ and $\mathbf{p}^{\prime} \in \mathbf{R}_{\eta}$ :

1. $\mathbf{p}^{\prime} \frown \mathbf{p} \Vdash \dot{h}(\vec{\nu}-\vec{\lambda})=\check{\imath}$ for some $i \in\{0,1\}$.

2. If $\mathbf{p}^{\prime \prime} \leq \mathbf{p}$ is any condition such that $\mathbf{p}^{\prime} \mathbf{p}^{\prime \prime} \Vdash \dot{h}(\vec{\nu}, \vec{\lambda})=\check{\imath}$ for some $i \in$ $\{0,1\}$, then $p$ has an instance of the recipe $P_{\gamma}$ at $\gamma=\inf ($ domain $\mathbf{p})$, and $\mathbf{p}^{\prime} \mathbf{p}^{\prime \prime} \uparrow \gamma \frown \mathbf{p} \Vdash \dot{h}(\vec{\nu} \frown \vec{\lambda})$.

The last expression is slightly sloppy, in that $\mathbf{p}$ should be modified so that $\lfloor\mathbf{p}\rfloor=$ $\left\lceil\mathbf{p}^{\prime \prime}\lceil\gamma\rceil\right.$.

We will maintain as a recursion hypothesis that, for any $\bar{\lambda}<\lambda_{0}^{n}$, the conditions $\mathbf{p}^{n}\left(\bar{\lambda}, \vec{\lambda}^{n}, \ldots, \vec{\lambda}^{1}\right)$ fully decide $h$ for $\vec{\lambda}=\left(\lambda_{0}^{n}, \ldots, \lambda_{0}^{1}\right)$ and, in addition, if $1 \leq i \leq n$ then the intermediate condition

$$
\tau_{i}^{n}\left(\lambda_{i}^{n}\right) \frown \ldots \frown \tau_{n}^{n}\left(\lambda_{n}^{n}\right) \frown \mathbf{p}^{n-1}\left(\left\lceil\tau_{n}^{n}\left(\lambda_{n}^{n}\right)\right\rceil, \vec{\lambda}^{n-1}, \ldots, \vec{\lambda}^{1}\right)
$$

fully decides $h$ for $\left\langle\lambda_{0}^{n-1}, \ldots, \lambda_{0}^{0}\right\rangle$.

We take $\mathbf{p}^{0}(\bar{\lambda})=\left(\kappa, \bar{\lambda}, \vec{B}^{0}\right)$ where the sequence $\vec{B}^{0}$ was defined at the beginning of the proof. Now suppose that the functions $\vec{B}^{k}$ and $\tau_{i}^{k}$ for $i \leq k<n$ have been defined so that $\mathbf{p}^{n-1}\left(\bar{\lambda}, \vec{\lambda}^{n-1}, \ldots, \vec{\lambda}^{1}\right)$ fully decides $h$ for $\vec{\lambda}=\left(\lambda_{0}^{n-1}, \ldots, \lambda_{0}^{1}\right)$.

First, taking $i=n$ in (19), use lemma 4.6 to define $\tau_{n}^{n}\left(\lambda_{n}^{n}\right)$ to be the least $\lambda_{n^{-}}^{n}$ closed condition in $\mathbf{R}_{\lambda_{n}^{n}, \lambda_{0}^{n-1}}$ such that (19) fully decides $h$ for $\vec{\lambda}$ for all $\bar{\lambda}<\lambda_{n}^{n}$. Now successively define the terms $\tau_{i}^{n}\left(\lambda_{i}^{n}\right) \leq^{*} \tau_{i}^{n-1}\left(\lambda_{i}^{n}\right)$, in decreasing order of the integers $i$ in the interval $n>i>0$, so that (19) continues to fully decide $h$ for $\vec{\lambda}$. This construction uses the fact that $\tau_{i}^{n-1}\left(\lambda_{i}^{n}\right)$ is a $\lambda_{i}^{n}$-closed condition. For each condition $\mathbf{p}^{\prime}$, the condition (19) will either satisfy clause 1 or will satisfy clause 2 vacuously.

Finally, complete the definition of $\mathbf{p}^{n}\left(\bar{\lambda}, \vec{\lambda}^{n}, \ldots, \vec{\lambda}^{1}\right)$ by defining $\vec{B}^{n}\left(\lambda_{0}^{n}\right)$ so that if $\bar{\lambda}<\lambda_{0}^{n}$ then $\mathbf{p}^{n}\left(\bar{\lambda}, \vec{\lambda}^{n}, \ldots, \vec{\lambda}^{1}\right)$ fully decides $h$ for $\left\langle\lambda_{0}^{n}, \ldots, \lambda_{0}^{1}\right\rangle$. The sequence $\vec{B}^{n}\left(\lambda_{0}^{n}\right)$ is constructed as a diagonal intersection $\triangle_{p, \vec{\nu}} \vec{B}_{p, \vec{\nu}}$ where $\vec{B}_{p, \vec{\nu}} \subset \vec{B}^{n-1} \uparrow \lambda_{0}^{n}$ is chosen so that the condition

$$
\mathbf{p}^{\frown}\left(\lambda_{0}^{n},\lceil\mathbf{p}\rceil, \vec{B}_{\overrightarrow{\mathbf{p}}, \vec{\nu}}\right) \frown \tau_{0}^{n}\left(\lambda_{0}^{n}\right) \frown \ldots \frown \tau_{n}^{n}\left(\lambda_{n}^{n}\right) \frown \mathbf{p}^{n-1}\left(\left\lceil\tau_{n}^{n}\left(\lambda_{n}^{n}\right)\right\rceil, \vec{\lambda}^{n-1}, \ldots, \vec{\lambda}^{n}\right)
$$

decides, if possible, the value of $h(\vec{\nu}, \vec{\lambda})$.

This completes the definition of the conditions $\tau_{i}^{n}\left(\lambda_{i}^{n}\right)$ and sequences $\vec{B}^{n}$, and hence of the conditions $t_{\gamma}$ and sequences $\vec{B}^{\omega}$. The definition of $\tau_{i}^{n}\left(\lambda_{i}^{n}\right)$ appears to depend on the larger ordinals in the sequence $\left\langle\vec{\lambda}^{n}, \ldots, \vec{\lambda}^{1}\right\rangle$; however because all of the ordinals $\lambda_{j}^{m}$ are members of $I$ and the definition can be carried out in $\mathcal{S}$, the 
normality of the set $I$ of indiscernibles implies that $\tau_{i}^{n}\left(\lambda_{i}^{n}\right)$ actually depends only on the ordinal $\lambda_{i}^{n}$.

This choice of $\tau_{i}^{n}(\lambda)$ and $\vec{B}^{n}$ ensure that the conditions $\mathbf{p}_{k}(\vec{\lambda}, \vec{\lambda}, \vec{\gamma})$ of (16) have the required properties, and hence the set $I^{*}$ constructed from them is a set of indiscernibles for $h$ in $V[C]$. Since $h$ was arbitrary, it follows that $\kappa$ is Ramsey in $V[C]$. This completes the proof of sufficiency of the condition for Ramsey cardinals, which is the last part of the proof of theorem 5.3.

Another direction in which the ideas of [2] and of this paper could be extended would be by strengthening the requirements on $C$. Replacing $E$ with a smaller class $E^{\prime}$ doesn't give anything new: It is straightforward to verify that the results hold true for any $E^{\prime} \subset E$, so long as $o(\lambda)$ is calculated using only measures $U$ such that $E^{\prime} \cap \operatorname{crit}(U) \in U$. A more ambitious idea would be to ask that $C$ be eventually contained in every member of an appropriate filter. The following theorem shows that this is routine, at least for Gitik's result about the preservation of an inaccessible cardinal, for the filter generated by the closed unbounded filter together with the set $E$ of inaccessible cardinals:

Theorem 5.7. If $\kappa \in D$ then there is a generic extension $V[C]$ of $V$ such that $\kappa$ is regular in $V[C]$ and $C$ is a closed and unbounded subset of $\kappa$ which contains only regular cardinals of $V$ and which is eventually contained in every closed, unbounded subset of $\kappa$ in $V$.

Proof. We use a modification $\mathcal{R}_{\kappa+1}^{\prime \prime}$ of the forcing $\mathcal{R}_{\kappa}^{\prime}$. Instead of using an instance of the recipe $P_{\kappa}$, we use a recipe $P_{\kappa}^{\prime \prime}$ which has instances of the form $(\kappa, c)$ where $c$ is a closed unbounded subset of $\kappa$. The ordering for $P_{\kappa}^{\prime}$ is the same as that for $P_{\kappa}$ in $\mathcal{R}_{\kappa}^{\prime}$ except for the added condition that $p^{\frown}\left(\kappa, c^{\prime}\right) \leq(\kappa, c)$ holds only when $c^{\prime} \subset c$ and $p \Vdash C_{p} \subset c$.

Clearly the result of this forcing is a closed and unbounded set $C \subset \kappa$ with the required property. Indeed, all of the arguments of section 3 can easily been seen to extend to this forcing, with the possible exception of lemma 3.1. Thus we will look at the proof of lemma 3.1 .

Let $(\kappa, c)$ be an arbitrary condition, and let $\left(\mathcal{D}_{\xi}: \xi<\tau\right)$ be a sequence of open dense subsets of $\mathcal{R}_{\tau, \kappa}^{\prime \prime}$. Let $\gamma$ and $Y_{\gamma}$ be such that $o(\gamma)=\tau, Y_{\gamma} \prec \mathrm{H}_{\kappa^{++}}$with $\left|Y_{\gamma}\right|=\gamma=\kappa \cap Y_{\gamma}$, and all of the relevant sets are members of $Y_{\gamma}$. We want to find a condition $(\gamma, A, h)^{\frown}\left(\kappa, c^{\prime}\right)$ such that if $\xi<\tau$ then $\left\{p \leq(\gamma, A, h): p^{\frown}\left(\kappa, c^{\prime}\right) \in \mathcal{D}_{\xi}\right\}$ is dense in $\mathcal{R}^{\prime \prime} /(\gamma, A, h)$.

For each $p \in \mathcal{R}_{\gamma}^{\prime \prime}$ there is a closed and unbounded subset $c_{p}$ of $\kappa$ so that for all $\xi<\tau$, either $p^{\frown}\left(\kappa, c_{p}\right) \in \mathcal{D}_{\xi}$ or else there is no closed and unbounded $c^{\prime} \subset \kappa$ so that $p^{\frown}\left(\kappa, c^{\prime}\right) \in \mathcal{D}_{\xi}$. Since $Y_{\gamma} \prec \mathrm{H}_{\kappa^{++}}$, we can take the function $p \mapsto c_{p}$ to be in $Y_{\gamma}$. Set $c^{\prime}=\triangle_{p \in \mathcal{R}_{\gamma}^{\prime \prime}} c_{p}$. Then $c^{\prime} \in Y_{\gamma}$ and $c^{\prime} \cap \gamma$ is a closed and unbounded subset of $\gamma$. Set $A=\left\{\nu \in c^{\prime} \cap \gamma: o(\gamma)<\tau\right\}$ and define $h$ in $Y_{\gamma}$ as in the proof of lemma 3.1 . so that for all $\nu \in A$,

$$
\Vdash_{\mathcal{R}_{\nu+1}^{\prime \prime}}\left(h(\nu) \subset c^{\prime} \text { and } \forall \xi<\tau \exists \bar{p} \in \mathfrak{G}(C \cap \nu+1) \exists \bar{c} \bar{p} \frown h(\nu) \frown(\kappa, \bar{c}) \in \mathcal{D}_{\xi}\right) .
$$

Then, just as in lemma 3.1 the condition $(\gamma,(A, h)) \frown\left(\kappa, c^{\prime}\right)$ forces that $\mathfrak{G}(C \cap \gamma) \cap \mathcal{D}_{\xi} \neq \varnothing$ for all $\xi<\tau$.

It is natural to ask whether theorem 1.1 can similarly be extended to give a model $V[C]$ in which $\kappa$ is measurable, $C \subset E$, and $C$ generates the closed unbounded filter 
of $V$. Radin forcing, using a stronger hypothesis, does provide such a model. In fact the set $C$ given by Radin forcing has the apparently stronger property that it generates the filter $\bigcap_{\beta<o(\kappa)} U(\kappa, \beta)$ of $V$.

A measure $U=U(\kappa, \delta)$ is called a weak repeat point if for all $B \in U$ there is $\beta<\delta$ such that $B \in U(\kappa, \beta)$. The following theorem is essentially due to Radin ([9], see also [8]):

Theorem 5.8. If there is a weak repeat point on $\kappa$ in $V$, then Radin forcing can be used to obtain a closed and unbounded set $C \subset \kappa$ so that $\kappa$ is still measurable in $V[C]$ and $C \backslash x$ is bounded for every set $x \in \bigcap_{\beta<o(\kappa)} U(\kappa, \beta)$.

The existence of a weak repeat point is weaker than $o(\kappa)=\kappa^{++}$, but much stronger than $o(\kappa)=\kappa^{+}$.

The following theorem shows that in this case the result using Radin forcing is the best possible: not only can the hypothesis not be weakened, but if $C$ generates the closed unbounded filter then it necessarily generates the filter $\bigcap_{\beta<o(\kappa)} U(\kappa, \beta)$ of $V$.

Theorem 5.9. If $\kappa$ is measurable and there is a closed unbounded subset $C$ of $\kappa$ which is almost contained in every closed unbounded set $X \in K$, then there is a weak repeat point on $\kappa$ in $K$.

Proof. The key observation is that, like a Radin generic set, $C$ is eventually contained in every set $X \in \bigcap_{\beta<o(\kappa)} U(\kappa, \beta)$. To see this, let $U$ be the measure on $\kappa$ in $V$ and let $i: V \rightarrow M=\operatorname{ult}(V, U)$. By the covering lemma, $i\left\lceil K: K \rightarrow K^{M}\right.$ is an iterated ultrapower, so let $I$ be the set of indiscernibles for measures on $i(\kappa)$ in $K^{M}$ generated by this iteration. Thus $I=\left\{j_{\alpha}(\kappa): \alpha<\theta\right\}$ where $\left(K_{\alpha}: \alpha<\theta\right)$ are the models in the iterated ultrapower and $j_{\alpha}: K=K_{0} \rightarrow K_{\alpha}$ are the associated embeddings, with $i\left\lceil K=j_{\theta}\right.$. Then $I \subset i(X)$ for any set $X \in \bigcap_{\beta<o(\kappa)} U(\kappa, \beta)$, so it is sufficient to show that $i(C) \backslash \kappa \subset I$. Suppose to the contrary that $\nu \in i(C) \backslash(\kappa \cup I)$. Then there is some $n<\omega$, some function $f:[\kappa]^{n} \rightarrow \kappa$ in $K$, and some sequence $\vec{c} \in[\nu]^{n}$ so that $\nu \leq i(f)(\vec{c})$.

Now let $X^{\prime}=\left\{\xi<\kappa: f^{\prime \prime}[\xi]^{n} \subset \xi\right\}$, so that $\nu \notin i\left(X^{\prime}\right)$. But $X^{\prime}$ is a closed and unbounded subset of $\kappa$, so $C \backslash X^{\prime}$ is bounded in $\kappa$ and hence $i(C) \backslash \kappa \subset i\left(X^{\prime}\right)$. This contradicts the assumption that $\nu \in i(C) \backslash X^{\prime}$, and this contradiction finishes the proof of the claim.

Now let $U(\kappa, \beta)$ be the first ultrafilter used in the iterated ultrapower, so that $U \cap K=U(\kappa, \beta)$. We now finish the proof of the theorem by showing that $U(\kappa, \beta)$ is a repeat point. Suppose to the contrary that $B \in U(\kappa, \beta)$ but $B \notin U\left(\kappa, \beta^{\prime}\right)$ for any $\beta^{\prime}<\beta$. By replacing $B$ if necessary with $B^{\prime}=\{\nu \in B: \forall \gamma<o(\nu) B \cap \nu \notin$ $U(\nu, \gamma)\} \in U(\kappa, \beta)$ we can assume that $B \notin U\left(\kappa, \beta^{\prime}\right)$ for all $\beta^{\prime} \neq \beta$. Furthermore $C \cap B$ is unbounded in $\kappa$, since $\kappa \in i(C) \cap i(B)=i(C \cap B)$. Now if $A \in \mathcal{P}^{K}(\kappa)$ then $A \in U(\kappa, \beta)$ if and only if $A \cup(\kappa \backslash B) \in \bigcap_{\gamma<o(\kappa)} U(\kappa, \gamma)$, and this holds if and only if $C \backslash(A \cup(\kappa \backslash B))$ is bounded in $\kappa$, that is, if and only if $C \cap B$ is eventually contained in $A$. Since $C \cap B \in M$ it follows that $U(\kappa, \beta) \in M$, and hence $U(\kappa, \beta) \in K^{M}$. This is impossible, since $U(\kappa, \beta) \notin K_{1}=\operatorname{ult}(K, U(\kappa, \beta))$, and this contradiction shows that $\beta$ is a repeat point.

\section{REFERENCES}

1. J. E. Baumgartner, L. A. Harrington, and E. M. Kleinberg, Adding a closed unbounded set, J. Symbolic Logic 41 (1976), no. 2, 481-482. MR 55:7782 
2. Moti Gitik, On closed unbounded sets consisting of former regulars, J. Symbolic Logic 64 (1999), no. 1, 1-12. MR 2000f:03151

3. William J. Mitchell, The covering lemma, In preparation; to appear in Handbook of Set Theory, M. Foreman, A. Kanimori and M. Magidor editors, Kluwer Press.

4. - Applications of the covering lemma for sequences of measures, Transactions of the American Mathematical Society 299 (1987), no. 1, 41-58. MR 88a:03122

5. - On the singular cardinal hypothesis, Transactions of the American Mathematical Society 329 (1992), no. 2, 507-530. MR 92e:03081

6. Jónsson cardinals, Erdös cardinals, and the core model, Journal of Symbolic Logic 64 (1999), no. 3, 1065-1086. CMP 2000:17

7. — A Gitik iteration with nearly Easton factoring, In preparation, 2001.

8. - One repeat point gives a closed, unbounded ultrafilter on $\omega_{1}$, In preparation, 2001.

9. Lon Berk Radin, Adding closed cofinal sequences to large cardinals, Ann. Math. Logic 22 (1982), no. 3, 243-261. MR 83m:03062

Department of Mathematics, University of Florida, Gainesville, Florida 32611

E-mail address: mitchell@math.ufl.edu 